\title{
Generalized logical operations among conditional events
}

\author{
Angelo Gilio $^{1} \cdot$ Giuseppe Sanfilippo $^{2}$ (D) \\ Published online: 27 July 2018 \\ (C) Springer Science+Business Media, LLC, part of Springer Nature 2018
}

\begin{abstract}
We generalize, by a progressive procedure, the notions of conjunction and disjunction of two conditional events to the case of $n$ conditional events. In our coherence-based approach, conjunctions and disjunctions are suitable conditional random quantities. We define the notion of negation, by verifying De Morgan's Laws. We also show that conjunction and disjunction satisfy the associative and commutative properties, and a monotonicity property. Then, we give some results on coherence of prevision assessments for some families of compounded conditionals; in particular we examine the Fréchet-Hoeffding bounds. Moreover, we study the reverse probabilistic inference from the conjunction $\mathcal{C}_{n+1}$ of $n+1$ conditional events to the family $\left\{\mathcal{C}_{n}, E_{n+1} \mid H_{n+1}\right\}$. We consider the relation with the notion of quasi-conjunction and we examine in detail the coherence of the prevision assessments related with the conjunction of three conditional events. Based on conjunction, we also give a characterization of p-consistency and of p-entailment, with applications to several inference rules in probabilistic nonmonotonic reasoning. Finally, we examine some non p-valid inference rules; then, we illustrate by an example two methods which allow to suitably modify non p-valid inference rules in order to get inferences which are p-valid.
\end{abstract}

Keywords Conditional events · Conditional random quantities · Conjunction · Disjunction · Negation · Fréchet-Hoeffding bounds · Coherent prevision assessments · Coherent extensions · Quasi conjunction · Probabilistic reasoning ·

p-entailment $\cdot$ Inference rules

\section{Introduction}

The research on combining logic and probability has a long history (see, e.g., $[2,8,13,16,33])$. In this paper we use a coherence-based approach to probability, which allows to introduce probability assessments on arbitrary families of conditional events, by properly managing conditioning events of zero probability (see, e.g., $[4,5,13,18,19,25$, $26,28,42])$. In probability theory and in probability logic a

Both authors contributed equally to this work.

This paper is a substantially extended version of [27].

Angelo Gilio is now retired.

Giuseppe Sanfilippo

giuseppe.sanfilippo@unipa.it

Angelo Gilio

angelo.gilio@sbai.uniroma1.it

1 Department SBAI, University of Rome "La Sapienza", Rome, Italy

2 Department of Mathematics and Computer Science, University of Palermo, Palermo, Italy relevant problem, largely discussed by many authors (see, e.g., $[3,14,15,32])$, is that of suitably defining logical operations among conditional events. In a pioneering paper, written in 1935 by de Finetti [16], it was proposed a threevalued logic for conditional events coinciding with that one of Lukasiewicz. A survey of the many contributions by different authors (such as Adams, Belnap, Calabrese, de Finetti, Dubois, van Fraassen, McGee, Goodmann, Lewis, Nguyen, Prade, Schay) to research on three-valued logics and compounds of conditionals has been given in [39]; conditionals have also been extensively studied in [17, 38]. In the literature, the conjunction and disjunction have been usually defined as suitable conditionals; see e.g. [2, 9, 11, 32]. A theory for the compounds of conditionals has been proposed in $[34,38]$. A related theory has been developed in the setting of coherence in $[22,23,26]$; in these papers, conjunction and disjunction of two conditional events are not defined as conditional events, but as suitable conditional random quantities, with values in the interval $[0,1]$. In the present paper we generalize the notions of conjunction and disjunction of two conditional events to the case of $n$ conditional events; we also give the notion of negation. Then, we examine a monotonicity 
property for conjunction and disjunction. Moreover, we give some results on coherence of prevision assessments for some families of compounded conditionals; in particular we examine the Fréchet-Hoeffding bounds. Finally, we examine in detail the coherence of prevision assessments related with the conjunction of three conditional events. The paper is organized as described below. In Section 2 we recall some preliminary notions and results which concern coherence, quasi conjunction, conjunction, disjunction, and Fréchet-Hoeffding bounds. In Section 3 we introduce, in a progressive way, the notions of conjunction and disjunction for $n$ conditional events; then, we define the notion of negation and we show that De Morgan's Laws are satisfied. We define the notion of conjunction (resp., disjunction) for the conjunctions (resp., disjunctions) associated with two families of conditional events, by showing then the validity of commutative and associative properties. In Section 4, after a preliminary result concerning the inequality $X|H \leq Y| K$ between two conditional random quantities, we show that the conjunction $\mathcal{C}_{n+1}$ of $n+1$ conditional events is a conditional random quantity less than or equal to any conjunction $\mathcal{C}_{n}$ of a subfamily of $n$ conditional events. Likewise, we show that the disjunction $\mathcal{D}_{n+1}$ of $n+1$ conditional events is greater than or equal to any disjunction $\mathcal{D}_{n}$ of a subfamily of $n$ conditional events. We also show that $\mathcal{C}_{n}$ and $\mathcal{D}_{n}$ belong to the interval $[0,1]$. Moreover, we derive some inequalities from the monotony property. In Section 5, based on a geometrical approach, we characterize by an iterative procedure the set of coherent assessments on the family $\left\{C_{n}, E_{n+1} \mid H_{n+1}, \mathcal{C}_{n+1}\right\}$. In Section 6 we study the (reverse) inference from $\mathcal{C}_{n+1}$ to $\left\{\mathcal{C}_{n}, E_{n+1} \mid H_{n+1}\right\}$, by determining the set of coherent extensions $\left(\mu_{n}, x_{n+1}\right)$ of any coherent assessment $\mu_{n+1}$, where $\mu_{n}=\mathbb{P}\left(\mathcal{C}_{n}\right)$, $x_{n+1}=P\left(E_{n+1} \mid H_{n+1}\right)$, and $\mu_{n+1}=\mathbb{P}\left(\mathcal{C}_{n+1}\right)$. In Section 7 we show that the prevision of the conjunction $\mathcal{C}_{n}$ satisfies the Fréchet-Hoeffding bounds. Then, by exploiting De Morgan's Laws, we give the dual result for the disjunction $\mathcal{D}_{n}$. In Section 8 we examine in detail the conjunction for a family of three conditional events $E_{1}\left|H_{1}, E_{2}\right| H_{2}, E_{3} \mid H_{3}$. We also consider the relation with the notion of quasi-conjunction studied in [2]; see also [24, 25]. We also determine the set of coherent prevision assessments on the whole family $\left\{E_{1}\left|H_{1}, E_{2}\right| H_{2}, E_{3} \mid H_{3},\left(E_{1} \mid H_{1}\right) \wedge\left(E_{2} \mid H_{2}\right),\left(E_{1} \mid H_{1}\right) \wedge\right.$ $\left(E_{3} \mid H_{3}\right),\left(E_{2} \mid H_{2}\right) \wedge\left(E_{3} \mid H_{3}\right),\left(E_{1} \mid H_{1}\right) \wedge\left(E_{2} \mid H_{2}\right) \wedge$ $\left.\left(E_{3} \mid H_{3}\right)\right\}$. Moreover, we consider the particular case where $H_{1}=H_{2}=H_{3}=H$. In Section 9, by applying our notion of conjunction, we give a characterization of p-consistency and p-entailment and we examine some p-valid inference rules in probabilistic nonmonotonic reasoning. Moreover, based on a suitable notion of iterated conditioning, we briefly describe a characterization of p-entailment in the case of two premises. In Section 10, after examining some non $\mathrm{p}$-valid inference rules, we illustrate two methods which allow to construct p-valid inferences. Finally, in Section 11 we give a summary of results. Notice that for almost all (new) results of this paper the proofs are given in Appendix A.

\section{Some preliminaries}

In this section we recall some basic notions and results on coherence (see, e.g., [5, 7, 10, 13, 40]). In our approach an event $A$ represents an uncertain fact described by a (non ambiguous) logical proposition; hence $A$ is a two-valued logical entity which can be true, or false. The indicator of $A$, denoted by the same symbol, is 1 , or 0 , according to whether $A$ is true, or false. The sure event is denoted by $\Omega$ and the impossible event is denoted by $\emptyset$. Moreover, we denote by $A \wedge B$, or simply $A B$, (resp., $A \vee B$ ) the logical conjunction (resp., logical disjunction). The negation of $A$ is denoted $\bar{A}$. Given any events $A$ and $B$, we simply write $A \subseteq B$ to denote that $A$ logically implies $B$, that is $A \bar{B}$ is the impossible event $\emptyset$. We recall that $n$ events are logically independent when the number $m$ of constituents, or possible worlds, generated by them is $2^{n}$ (in general $m \leq 2^{n}$ ).

\subsection{Conditional events and coherent probability assessments}

Given two events $E, H$, with $H \neq \emptyset$, the conditional event $E \mid H$ is defined as a three-valued logical entity which is true, or false, or void, according to whether $E H$ is true, or $\bar{E} H$ is true, or $\bar{H}$ is true, respectively. We recall that, agreeing to the betting metaphor, if you assess $P(E \mid H)=$ $p$, then, for every real number $s$, you are willing to pay an amount $p s$ and to receive $s$, or 0 , or $p s$, according to whether $E H$ is true, or $\bar{E} H$ is true, or $\bar{H}$ is true (bet called off), respectively. Then, the random gain associated with the assessment $P(E \mid H)=p$ is $G=s H E+p s \bar{H}-p s=$ $s H(E-p)$. Given a real function $P: \mathcal{K} \rightarrow \mathcal{R}$, where $\mathcal{K}$ is an arbitrary family of conditional events, let us consider a subfamily $\mathcal{F}=\left\{E_{1}\left|H_{1}, \ldots, E_{n}\right| H_{n}\right\}$ of $\mathcal{K}$ and the vector $\mathcal{P}=\left(p_{1}, \ldots, p_{n}\right)$, where $p_{i}=P\left(E_{i} \mid H_{i}\right), \quad i \in J_{n}=$ $\{1, \ldots, n\}$. We denote by $\mathcal{H}_{n}$ the disjunction $H_{1} \vee \cdots \vee H_{n}$. As $E_{i} H_{i} \vee \bar{E}_{i} H_{i} \vee \bar{H}_{i}=\Omega, \quad i \in J_{n}$, by expanding the expression $\bigwedge_{i \in J_{n}}\left(E_{i} H_{i} \vee \bar{E}_{i} H_{i} \vee \bar{H}_{i}\right)$ we can represent $\Omega$ as the disjunction of $3^{n}$ logical conjunctions, some of which may be impossible. The remaining ones are the constituents generated by $\mathcal{F}$ and, of course, are a partition of $\Omega$. We denote by $C_{1}, \ldots, C_{m}$ the constituents which logically imply $\mathcal{H}_{n}$ and (if $\mathcal{H}_{n} \neq \Omega$ ) by $C_{0}$ the remaining constituent $\overline{\mathcal{H}}_{n}=\bar{H}_{1} \cdots \bar{H}_{n}$, so that

$$
\begin{aligned}
\mathcal{H}_{n} & =C_{1} \vee \cdots \vee C_{m}, \\
\Omega & =\overline{\mathcal{H}}_{n} \vee \mathcal{H}_{n}=C_{0} \vee C_{1} \vee \cdots \vee C_{m}, \quad m+1 \leq 3^{n} .
\end{aligned}
$$


In the context of betting scheme, with the pair $(\mathcal{F}, \mathcal{P})$ we associate the random gain $G=\sum_{i \in J_{n}} s_{i} H_{i}\left(E_{i}-p_{i}\right)$, where $s_{1}, \ldots, s_{n}$ are $n$ arbitrary real numbers. We observe that $G$ is the difference between the amount that you receive, $\sum_{i \in J_{n}} s_{i}\left(E_{i} H_{i}+p_{i} \bar{H}_{i}\right)$, and the amount that you pay, $\sum_{i \in J_{n}} s_{i} p_{i}$, and represents the net gain from engaging each transaction $H_{i}\left(E_{i}-p_{i}\right)$, the scaling and meaning (buy or sell) of the transaction being specified by the magnitude and the sign of $s_{i}$, respectively. Let $g_{h}$ be the value of $G$ when $C_{h}$ is true; then $G \in\left\{g_{0}, g_{1}, \ldots, g_{m}\right\}$. Of course, $g_{0}=0$. We denote by $\mathcal{G}_{\mathcal{H}_{n}}$ the set of values of $G$ restricted to $\mathcal{H}_{n}$, that is $\mathcal{G}_{\mathcal{H}_{n}}=\left\{g_{1}, \ldots, g_{m}\right\}$. Then, based on the betting scheme of de Finetti, we have

Definition 1 The function $P$ defined on $\mathcal{K}$ is said to be coherent if and only if, for every integer $n$, for every finite subfamily $\mathcal{F}$ of $\mathcal{K}$ and for every real numbers $s_{1}, \ldots, s_{n}$, one has: $\min \mathcal{G}_{\mathcal{H}_{n}} \leq 0 \leq \max \mathcal{G}_{\mathcal{H}_{n}}$.

Notice that the condition $\min \mathcal{G}_{\mathcal{H}_{n}} \leq 0 \leq \max \mathcal{G}_{\mathcal{H}_{n}}$ can be written in two equivalent ways: $\min \mathcal{G}_{\mathcal{H}_{n}} \leq 0$, or $\max \mathcal{G}_{\mathcal{H}_{n}} \geq 0$. As shown by Definition 1, a probability assessment is coherent if and only if, in any finite combination of $n$ bets, it does not happen that the values $g_{1}, \ldots, g_{m}$ are all positive, or all negative (no Dutch Book).

\subsection{Coherent conditional prevision assessments}

Given a prevision function $\mathbb{P}$ defined on an arbitrary family $\mathcal{K}$ of finite conditional random quantities, consider a finite subfamily $\mathcal{F}=\left\{X_{1}\left|H_{1}, \ldots, X_{n}\right| H_{n}\right\} \subseteq \mathcal{K}$ and the vector $\mathcal{M}=\left(\mu_{1}, \ldots, \mu_{n}\right)$, where $\mu_{i}=\mathbb{P}\left(X_{i} \mid H_{i}\right)$ is the assessed prevision for the conditional random quantity $X_{i} \mid H_{i}, i \in J_{n}$. With the pair $(\mathcal{F}, \mathcal{M})$ we associate the random gain $G=$ $\sum_{i \in J_{n}} s_{i} H_{i}\left(X_{i}-\mu_{i}\right)$; moreover, we denote by $\mathcal{G}_{\mathcal{H}_{n}}$ the set of values of $G$ restricted to $\mathcal{H}_{n}=H_{1} \vee \cdots \vee H_{n}$. Then, by the betting scheme, we have

Definition 2 The function $\mathbb{P}$ defined on $\mathcal{K}$ is coherent if and only if, $\forall n \geq 1, \forall \mathcal{F} \subseteq \mathcal{K}, \forall s_{1}, \ldots, s_{n} \in \mathbb{R}$, it holds that: $\min \mathcal{G}_{\mathcal{H}_{n}} \leq 0 \leq \max \mathcal{G}_{\mathcal{H}_{n}}$.

Given a family $\mathcal{F}=\left\{X_{1}\left|H_{1}, \ldots, X_{n}\right| H_{n}\right\}$, for each $i \in J_{n}$ we denote by $\left\{x_{i 1}, \ldots, x_{i r_{i}}\right\}$ the set of possible values for the restriction of $X_{i}$ to $H_{i}$; then, for each $i \in J_{n}$ and $j=$ $1, \ldots, r_{i}$, we set $A_{i j}=\left(X_{i}=x_{i j}\right)$. Of course, for each $i \in$ $J_{n}$, the family $\left\{\bar{H}_{i}, A_{i j} H_{i}, j=1, \ldots, r_{i}\right\}$ is a partition of the sure event $\Omega$, with $A_{i j} H_{i}=A_{i j}, \bigvee_{j=1}^{r_{i}} A_{i j}=H_{i}$. Then, the constituents generated by the family $\mathcal{F}$ are (the elements of the partition of $\Omega$ ) obtained by expanding the expression $\bigwedge_{i \in J_{n}}\left(A_{i 1} \vee \cdots \vee A_{i r_{i}} \vee \bar{H}_{i}\right)$. We set $C_{0}=\bar{H}_{1} \cdots \bar{H}_{n}$ (it may be $C_{0}=\emptyset$ ); moreover, we denote by $C_{1}, \ldots, C_{m}$ the constituents contained in $\mathcal{H}_{n}$. Hence $\bigwedge_{i \in J_{n}}\left(A_{i 1} \vee \cdots \vee\right.$
$\left.A_{i r_{i}} \vee \bar{H}_{i}\right)=\bigvee_{h=0}^{m} C_{h}$. With each $C_{h}, h \in J_{m}$, we associate a vector $Q_{h}=\left(q_{h 1}, \ldots, q_{h n}\right)$, where $q_{h i}=x_{i j}$ if $C_{h} \subseteq A_{i j}, j=1, \ldots, r_{i}$, while $q_{h i}=\mu_{i}$ if $C_{h} \subseteq \bar{H}_{i}$; with $C_{0}$ it is associated $Q_{0}=\mathcal{M}=\left(\mu_{1}, \ldots, \mu_{n}\right)$. Denoting by $\mathcal{I}$ the convex hull of $Q_{1}, \ldots, Q_{m}$, the condition $\mathcal{M} \in \mathcal{I}$ amounts to the existence of a vector $\left(\lambda_{1}, \ldots, \lambda_{m}\right)$ such that: $\sum_{h \in J_{m}} \lambda_{h} Q_{h}=\mathcal{M}, \sum_{h \in J_{m}} \lambda_{h}=1, \lambda_{h} \geq 0, \forall h$; in other words, $\mathcal{M} \in \mathcal{I}$ is equivalent to the solvability of the system $(\Sigma)$, associated with $(\mathcal{F}, \mathcal{M})$,

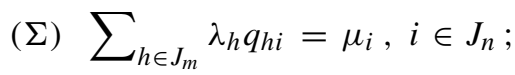

$$
\begin{aligned}
& \sum_{h \in J_{m}} \lambda_{h}=1 ; \lambda_{h} \geq 0, h \in J_{m} .
\end{aligned}
$$

Given the assessment $\mathcal{M}=\left(\mu_{1}, \ldots, \mu_{n}\right)$ on $\mathcal{F}=$ $\left\{X_{1}\left|H_{1}, \ldots, X_{n}\right| H_{n}\right\}$, let $S$ be the set of solutions $\Lambda=$ $\left(\lambda_{1}, \ldots, \lambda_{m}\right)$ of system $(\Sigma)$ defined in (1). Then, the following characterization theorem for coherent assessments on finite families of conditional events can be proved [6]

Theorem 1 (Characterization of coherence) Given a family of $n$ conditional random quantities $\mathcal{F}=$ $\left\{X_{1}\left|H_{1}, \ldots, X_{n}\right| H_{n}\right\}$, with finite sets of possible values, and a vector $\mathcal{M}=\left(\mu_{1}, \ldots, \mu_{n}\right)$, the conditional prevision assessment $\mathbb{P}\left(X_{1} \mid H_{1}\right)=\mu_{1}, \ldots, \mathbb{P}\left(X_{n} \mid H_{n}\right)=\mu_{n}$ is coherent if and only if, for every subset $J \subseteq J_{n}$, defining $\mathcal{F}_{J}=\left\{X_{i} \mid H_{i}, i \in J\right\}, \mathcal{M}_{J}=\left(\mu_{i}, i \in J\right)$, the system $\left(\Sigma_{J}\right)$ associated with the pair $\left(\mathcal{F}_{J}, \mathcal{M}_{J}\right)$ is solvable.

We point out that the solvability of system $(\Sigma)$ (i.e., the condition $\mathcal{M} \in \mathcal{I}$ ) is a necessary (but not sufficient) condition for coherence of $\mathcal{M}$ on $\mathcal{F}$. Moreover, assuming the system $(\Sigma)$ solvable, that is $S \neq \emptyset$, we define:

$$
I_{0}=\left\{i: \max _{\Lambda \in S} \sum_{h: C_{h} \subseteq H_{i}} \lambda_{h}=0\right\}, \begin{aligned}
& \mathcal{F}_{0}=\left\{X_{i} \mid H_{i}, i \in I_{0}\right\}, \\
& \mathcal{M}_{0}=\left(\mu_{i}, i \in I_{0}\right) .
\end{aligned}
$$

Then, the following theorem can be proved [6, Theorem 3]

Theorem 2 (Operative characterization of coherence) $A$ conditional prevision assessment $\mathcal{M}=\left(\mu_{1}, \ldots, \mu_{n}\right)$ on the family $\mathcal{F}=\left\{X_{1}\left|H_{1}, \ldots, X_{n}\right| H_{n}\right\}$ is coherent if and only if the following conditions are satisfied:

(i) the system $(\Sigma)$ defined in (1) is solvable;

(ii) if $I_{0} \neq \emptyset$, then $\mathcal{M}_{0}$ is coherent.

By Theorem 2, the following algorithm checks in a finite number of steps the coherence of the prevision assessment $\mathcal{M}$ on $\mathcal{F}$.

Algorithm 1 Let be given the pair $(\mathcal{F}, \mathcal{M})$.

1. Construct the system $(\Sigma)$ defined in (1) and check its solvability; 
2. If the system $(\Sigma)$ is not solvable then $\mathcal{M}$ is not coherent and the procedure stops, otherwise compute the set $I_{0}$;

3. If $I_{0}=\varnothing$ then $\mathcal{M}$ is coherent and the procedure stops, otherwise set $(\mathcal{F}, \mathcal{M})=\left(\mathcal{F}_{0}, M_{0}\right)$ and repeat steps $1-3$.

By following the approach given in $[12,22,23,26,29$, 35] a conditional random quantity $X \mid H$ can be seen as the random quantity $X H+\mu \bar{H}$, where $\mu=\mathbb{P}(X \mid H)$. In particular, in numerical terms, $A \mid H$ is the random quantity $A H+x \bar{H}$, where $x=P(A \mid H)$. Then, when $H \subseteq A$, coherence requires that $P(A \mid H)=1$ and hence $A \mid H=$ $H+\bar{H}=1$. Notice that, as $\bar{H} \mid H=0$ and $X H|H=X| H$, it holds that: $(X H+\mu \bar{H})|H=X H| H+\mu \bar{H}|H=X| H$, where $\mu=\mathbb{P}(X \mid H)$. Moreover, the negation of $A \mid H$ is defined as $\overline{A \mid H}=1-A|H=\bar{A}| H$. Coherence can be characterized in terms of proper scoring rules [7, 21], which can be related to the notion of entropy in information theory $[36,37]$.

We recall a result (see [26, Theorem 4]) which shows that, given two conditional random quantities $X|H, Y| K$, if $X|H=Y| K$ when $H \vee K$ is true, then $X|H=Y| K$ also when $H \vee K$ is false, so that $X|H=Y| K$.

Theorem 3 Given any events $H \neq \emptyset, K \neq \emptyset$, and any r.q.'s $X, Y$, let $\Pi$ be the set of the coherent prevision assessments $\mathbb{P}(X \mid H)=\mu, \mathbb{P}(Y \mid K)=v$.

(i) Assume that, for every $(\mu, v) \in \Pi, X|H=Y| K$ when $H \vee K$ is true; then $\mu=v$ for every $(\mu, v) \in \Pi$.

(ii) For every $(\mu, v) \in \Pi, X|H=Y| K$ when $H \vee K$ is true if and only if $X|H=Y| K$.

\subsection{Quasi conjunction, conjunction, and disjunction of two conditional events}

The notion of quasi conjunction plays an important role in nonmonotonic reasoning. In particular for two conditional events $A|H, B| K$ the quasi conjunction $Q C(A|H, B| K)$ is the conditional event $(\bar{H} \vee A) \wedge(\bar{K} \vee B) \mid(H \vee K)$. Note that: $Q C(A|H, B| K)$ is true, when a conditional event is true and the other one is not false; $Q C(A|H, B| K)$ is false, when a conditional event is false; $Q C(A|H, B| K)$ is void, when $H \vee K$ is false. In other words, the quasi conjunction is the conjunction of the two material conditionals $\bar{H} \vee A, \bar{K} \vee B$ given the disjunction of the conditioning events $H, K$. In numerical terms one has

$Q C(A|H, B| K)=\min \{\bar{H} \vee A, \bar{K} \vee B\} \mid(H \vee K)$

and, if we replace the material conditionals $\bar{H} \vee A, \bar{K} \vee B$ by the conditional events $A|H, B| K$, from formula (3) we obtain the definition below [23].
Definition 3 Given any pair of conditional events $A \mid H$ and $B \mid K$, with $P(A \mid H)=x, P(B \mid K)=y$, we define their conjunction as the conditional random quantity $(A \mid H) \wedge$ $(B \mid K)=Z \mid(H \vee K)$, where $Z=\min \{A|H, B| K\}$.

Then, defining $z=\mathbb{P}[(A \mid H) \wedge(B \mid K)]$, we have

$(A \mid H) \wedge(B \mid K)= \begin{cases}1, & \text { if } A H B K \text { is true, } \\ 0, & \text { if } \bar{A} H \vee \bar{B} K \text { is true, } \\ x, & \text { if } \bar{H} B K \text { is true, } \\ y, & \text { if } A H \bar{K} \text { is true, } \\ z, & \text { if } \bar{H} \bar{K} \text { is true. }\end{cases}$

Remark 1 We recall that $A \mid H=A H+x \bar{H}$, where $x=$ $P(A \mid H)$. Then, by Definition 3 , it holds that

$(A \mid H) \wedge(A \mid H)=(A \mid H)|H=(A H+x \bar{H})| H=A H|H=A| H$.

From (4), the conjunction $(A \mid H) \wedge(B \mid K)$ is the following random quantity

$(A \mid H) \wedge(B \mid K)=1 \cdot A H B K+x \cdot \bar{H} B K+y \cdot A H \bar{K}+z \cdot \bar{H} \bar{K}$.

For the quasi conjunction it holds that

$Q C(A|H, B| K)=A H B K+\bar{H} B K+A H \bar{K}+v \cdot \bar{H} \bar{K}$,

where $v=P(Q C(A|H, B| K))$. We recall that, if $P(A \mid H)=P(B \mid K)=1$, then $v=1$ (see, e.g., [25, Section 3]). We also recall a result which shows that Fréchet-Hoeffding bounds still hold for the conjunction of conditional events [26, Theorem 7].

Theorem 4 Given any coherent assessment $(x, y)$ on $\{A|H, B| K\}$, with $A, H, B, K$ logically independent, $H \neq$ $\emptyset, K \neq \emptyset$, the extension $z=\mathbb{P}[(A \mid H) \wedge(B \mid K)]$ is coherent if and only if the following Fréchet-Hoeffding bounds are satisfied: $\max \{x+y-1,0\}=z^{\prime} \leq z \leq z^{\prime \prime}=\min \{x, y\}$.

Remark 2 We observe that, if $x=y=1$, then coherence requires that $z=v=1$ and then by (5) and (6) it follows that $(A \mid H) \wedge(B \mid K)=Q C(A|H, B| K)$.

We recall now the notion of disjunction of two conditional events.

Definition 4 Given any pair of conditional events $A \mid H$ and $B \mid K$, with $P(A \mid H)=x, P(B \mid K)=y$, we define their disjunction as $(A \mid H) \vee(B \mid K)=W \mid(H \vee K)$, where $W=\max \{A|H, B| K\}$. 
Then, defining $w=\mathbb{P}[(A \mid H) \vee(B \mid K)]$, we have

$(A \mid H) \vee(B \mid K)= \begin{cases}1, & \text { if } A H \vee B K \text { is true, } \\ 0, & \text { if } \bar{A} H \bar{B} K \text { is true, } \\ x, & \text { if } \bar{H} \bar{B} K \text { is true, } \\ y, & \text { if } \bar{A} H \bar{K} \text { is true, } \\ w, & \text { if } \bar{H} \bar{K} \text { is true. }\end{cases}$

Remark 3 We recall that $A \mid H=A H+x \bar{H}$, where $x=$ $P(A \mid H)$. Then, by Definition 4, it holds that

$(A \mid H) \vee(A \mid H)=(A \mid H)|H=(A H+x \bar{H})| H=A H|H=A| H$.

From (7), the disjunction $(A \mid H) \vee(B \mid K)$ is the following random quantity

$$
(A \mid H) \vee(B \mid K)=1 \cdot A H \vee B K+x \cdot \bar{H} \bar{B} K+y \cdot \bar{A} H \bar{K}+w \cdot \bar{H} \bar{K}
$$

\section{Conjunction, disjunction, and negation}

We now define the conjunction and the disjunction of $n$ conditional events in a progressive way by specifying the possible values of the corresponding conditional random quantities. Given a family of $n$ conditional events $\mathcal{F}=$ $\left\{E_{1}\left|H_{1}, \ldots, E_{n}\right| H_{n}\right\}$, we denote by $C_{0}, C_{1}, \ldots, C_{m}$, with $m+1 \leq 3^{n}$, the constituents associated with $\mathcal{F}$, where $C_{0}=\bar{H}_{1} \bar{H}_{2} \cdots \bar{H}_{n}$. With each $C_{h}, h=0,1, \ldots, m$, we associate a tripartition $\left(S_{h}^{\prime}, S_{h}^{\prime \prime}, S_{h}^{\prime \prime \prime}\right)$ of the set $\{1, \ldots, n\}$, such that, for each $i \in\{1, \ldots, n\}$ it holds that: $i \in S_{h}^{\prime}$, or $i \in S_{h}^{\prime \prime}$, or $i \in S_{h}^{\prime \prime \prime}$, according to whether $C_{h} \subseteq E_{i} H_{i}$, or $C_{h} \subseteq \bar{E}_{i} H_{i}$, or $C_{h} \subseteq \bar{H}_{i}$. In other words, for each $h=0,1, \ldots, m$, we have

$S_{h}^{\prime}=\left\{i: C_{h} \subseteq E_{i} H_{i}\right\}, S_{h}^{\prime \prime}=\left\{i: C_{h} \subseteq \bar{E}_{i} H_{i}\right\}$,

$S_{h}^{\prime \prime \prime}=\left\{i: C_{h} \subseteq \bar{H}_{i}\right\}$.

Definition 5 (Conjunction of $n$ conditionals) Let be given a family of $n$ conditional events $\mathcal{F}=\left\{E_{1}\left|H_{1}, \ldots, E_{n}\right| H_{n}\right\}$. For each non-empty subset $S$ of $\{1, \ldots, n\}$, let $x_{S}$ be a prevision assessment on $\bigwedge_{i \in S}\left(E_{i} \mid H_{i}\right)$. Then, the conjunction $\mathcal{C}(\mathcal{F})=\left(E_{1} \mid H_{1}\right) \wedge \cdots \wedge\left(E_{n} \mid H_{n}\right)$ is defined as

$$
\begin{aligned}
& Z_{n} \mid\left(H_{1} \vee \cdots \vee H_{n}\right)=\sum_{h=0}^{m} z_{h} C_{h}, \\
& \text { where } z_{h}= \begin{cases}1, & \text { if } S_{h}^{\prime}=\{1, \ldots, n\}, \\
0, & \text { if } S_{h}^{\prime \prime} \neq \emptyset, \\
x_{S_{h}^{\prime \prime \prime}}, & \text { if } S_{h}^{\prime \prime}=\emptyset \text { and } S_{h}^{\prime \prime \prime} \neq \emptyset .\end{cases}
\end{aligned}
$$

Remark 4 As shown by (10), the conjunction $\left(E_{1} \mid H_{1}\right) \wedge$ $\cdots \wedge\left(E_{n} \mid H_{n}\right)$ assumes one of the following possible values: 1 , when every conditional event is true; 0 , when at least one conditional event is false; $x_{S}$, when the conditional event $E_{i} \mid H_{i}$ is void, for every $i \in S$, and is true for every $i \notin S$. In the case $S=\{i\}$, we simply set $x_{S}=x_{i}$.

Notice that the notion of conjunction given in (10) has been already proposed, with positive probabilities for the conditioning events, in [38]. But, our approach is developed in the setting of coherence, where conditional probabilities and conditional previsions are primitive notions. Moreover, coherence allows to properly manage zero probabilities for conditioning events.

Remark 5 We observe that to introduce the random quantity defined by formula (10) we need to specify in a coherent way the set of prevision assessments $\left\{x_{S}: S \subseteq\right.$ $\{1,2, \ldots, n\}\}$. In particular, when the conditioning events $H_{1}, \ldots, H_{n}$ are all false, i.e. $C_{0}$ is true, the associated tripartition is $\left(S_{0}^{\prime}, S_{0}^{\prime \prime}, S_{0}^{\prime \prime \prime}\right)=(\emptyset, \emptyset,\{1,2, \ldots, n\})$ and the value of the conjunction $\mathcal{C}(\mathcal{F})$ is its prevision $x_{S_{0}^{\prime \prime \prime}}=$ $\mathbb{P}[\mathcal{C}(\mathcal{F})]$. Moreover, we observe that the set of the constituents $\left\{C_{0}, \ldots, C_{m}\right\}$ associated with $\mathcal{F}$ is invariant with respect to any permutation of the conditional events in $\mathcal{F}$. Then, the operation of conjunction introduced by Definition 5 is invariant with respect to any permutation of the conditional events in $\mathcal{F}$.

Definition 6 Given two finite families of conditional events $\mathcal{F}^{\prime}$ and $\mathcal{F}^{\prime \prime}$, based on Definition 5, we set $\mathcal{C}\left(\mathcal{F}^{\prime}\right) \wedge \mathcal{C}\left(\mathcal{F}^{\prime \prime}\right)=$ $\mathcal{C}\left(\mathcal{F}^{\prime} \cup \mathcal{F}^{\prime \prime}\right)$.

Proposition 1 The operation of conjunction is associative and commutative.

Proof Concerning the commutative property, let be given two finite families of conditional events $\mathcal{F}^{\prime}$ and $\mathcal{F}^{\prime \prime}$. As $\mathcal{F}^{\prime \prime} \cup \mathcal{F}^{\prime}=\mathcal{F}^{\prime} \cup \mathcal{F}^{\prime \prime}$, it holds that $\mathcal{C}\left(\mathcal{F}^{\prime \prime}\right) \wedge \mathcal{C}\left(\mathcal{F}^{\prime}\right)=$ $\mathcal{C}\left(\mathcal{F}^{\prime \prime} \cup \mathcal{F}^{\prime}\right)=\mathcal{C}\left(\mathcal{F}^{\prime} \cup \mathcal{F}^{\prime \prime}\right)=\mathcal{C}\left(\mathcal{F}^{\prime}\right) \wedge \mathcal{C}\left(\mathcal{F}^{\prime \prime}\right)$. Concerning the associative property, let be given three finite families of conditional events $\mathcal{F}^{\prime}, \mathcal{F}^{\prime \prime}$ and $\mathcal{F}^{\prime \prime \prime}$. We have

$$
\begin{aligned}
& {\left[\mathcal{C}\left(\mathcal{F}^{\prime}\right) \wedge \mathcal{C}\left(\mathcal{F}^{\prime \prime}\right)\right] \wedge \mathcal{C}\left(\mathcal{F}^{\prime \prime \prime}\right)=\mathcal{C}\left(\mathcal{F}^{\prime} \cup \mathcal{F}^{\prime \prime}\right) \wedge \mathcal{C}\left(\mathcal{F}^{\prime \prime \prime}\right)} \\
& \quad=\mathcal{C}\left(\mathcal{F}^{\prime} \cup \mathcal{F}^{\prime \prime} \cup \mathcal{F}^{\prime \prime \prime}\right)=\mathcal{C}\left(\mathcal{F}^{\prime}\right) \wedge \mathcal{C}\left(\mathcal{F}^{\prime \prime} \cup \mathcal{F}^{\prime \prime \prime}\right) \\
& \quad=\mathcal{C}\left(\mathcal{F}^{\prime}\right) \wedge\left[\mathcal{C}\left(\mathcal{F}^{\prime \prime}\right) \wedge \mathcal{C}\left(\mathcal{F}^{\prime \prime \prime}\right)\right]=\mathcal{C}\left(\mathcal{F}^{\prime}\right) \wedge \mathcal{C}\left(\mathcal{F}^{\prime \prime}\right) \wedge \mathcal{C}\left(\mathcal{F}^{\prime \prime \prime}\right)
\end{aligned}
$$

Definition 7 (Disjunction of $n$ conditionals) Let be given a family of $n$ conditional events $\mathcal{F}=\left\{E_{1}\left|H_{1}, \ldots, E_{n}\right| H_{n}\right\}$. Morever, for each non-empty subset $S$ of $\{1, \ldots, n\}$, let $y_{S}$ be a prevision assessment on $\bigvee_{i \in S}\left(E_{i} \mid H_{i}\right)$. 
Then, the disjunction $\mathcal{D}(\mathcal{F})=\left(E_{1} \mid H_{1}\right) \vee \cdots \vee\left(E_{n} \mid H_{n}\right)$ is defined as the following conditional random quantity

$W_{n} \mid\left(H_{1} \vee \cdots \vee H_{n}\right)=\sum_{h=0}^{m} w_{h} C_{h}$,

where $w_{h}= \begin{cases}1, & \text { if } S_{h}^{\prime} \neq \emptyset, \\ 0, & \text { if } S_{h}^{\prime \prime}=\{1,2, \ldots, n\}, \\ y_{S_{h}^{\prime \prime \prime}}, & \text { if } S_{h}^{\prime}=\emptyset \text { and } S_{h}^{\prime \prime \prime} \neq \emptyset .\end{cases}$

We recall that $S_{0}^{\prime \prime \prime}=\{1,2, \ldots, n\}$; thus $y_{S_{0}^{\prime \prime \prime}}=\mathbb{P}\left[\bigvee_{i=1}^{n}\right.$ $\left.\left(E_{i} \mid H_{i}\right)\right]=\mathbb{P}[\mathcal{D}(\mathcal{F})]$. As shown by $(11)$, the disjunction $\mathcal{D}(\mathcal{F})$ assumes one of the following possible values: 1, when at least one conditional event is true; 0 , when every conditional event is false; $y_{S}$, when the conditional event $E_{i} \mid H_{i}$ is void, for every $i \in S$, and is false for every $i \notin S$.

Definition 8 Given two finite families of conditional events $\mathcal{F}^{\prime}$ and $\mathcal{F}^{\prime \prime}$, based on Definition 7 , we set $\mathcal{D}\left(\mathcal{F}^{\prime}\right) \vee \mathcal{D}\left(\mathcal{F}^{\prime \prime}\right)=$ $\mathcal{D}\left(\mathcal{F}^{\prime} \cup \mathcal{F}^{\prime \prime}\right)$.

Proposition 2 The operation of disjunction is associative and commutative.

Proof The proof is analogous to that of Proposition 1.

We give below the notion of negation for the conjunction and the disjunction of a family of conditional events.

Definition 9 Given a family of conditional events $\mathcal{F}$, the negations for the conjunction $\mathcal{C}(\mathcal{F})$ and the disjunction $\mathcal{D}(\mathcal{F})$ are defined as $\overline{\mathcal{C}(\mathcal{F})}=1-\mathcal{C}(\mathcal{F})$ and $\overline{\mathcal{D}(\mathcal{F})}=$ $1-\mathcal{D}(\mathcal{F})$, respectively.

Given a family of $n$ conditional events $\mathcal{F}=\left\{E_{1} \mid H_{1}, \ldots\right.$, $\left.E_{n} \mid H_{n}\right\}$, we denote by $\overline{\mathcal{F}}$ the family $\left\{\bar{E}_{1}\left|H_{1}, \ldots, \bar{E}_{n}\right| H_{n}\right\}$. Of course $\overline{\overline{\mathcal{F}}}=\mathcal{F}$. In the next result we show that De Morgan's Laws are satisfied.

Theorem 5 Given a family of $n$ conditional events $\mathcal{F}=$ $\left\{E_{1}\left|H_{1}, \ldots, E_{n}\right| H_{n}\right\}$, it holds that:

(i) $\overline{\mathcal{D}(\mathcal{F})}=\mathcal{C}(\overline{\mathcal{F}})$, that is $\mathcal{D}(\mathcal{F})=\overline{\mathcal{C}(\overline{\mathcal{F}})}$;

(ii) $\overline{\mathcal{C}(\mathcal{F})}=\mathcal{D}(\overline{\mathcal{F}})$, that is $\mathcal{C}(\mathcal{F})=\overline{\mathcal{D}(\overline{\mathcal{F}})}$.

Proof See Appendix A.

\section{Monotonicity property}

For any given $n$ conditional events $E_{1}\left|H_{1}, \ldots, E_{n}\right| H_{n}$, we set $\mathcal{C}_{n}=\bigwedge_{i=1}^{n}\left(E_{i} \mid H_{i}\right)$ and $\mathcal{D}_{n}=\bigvee_{i=1}^{n}\left(E_{i} \mid H_{i}\right)$.
Moreover, for every non empty subset $S$ of $\{1,2, \ldots, n\}$ we set

$\mathcal{C}_{S}=\bigwedge_{i \in S}\left(E_{i} \mid H_{i}\right), \quad \mathcal{D}_{S}=\bigvee_{i \in S}\left(E_{i} \mid H_{i}\right)$.

In this section, among other results, we will show the monotonicity property of conjunction and disjunction, that is $\mathcal{C}_{n+1} \leq \mathcal{C}_{n}$ and $\mathcal{D}_{n+1} \geq \mathcal{D}_{n}$, for every $n \geq 1$.

We first prove a preliminary result, which in particular shows that, given two conditional random quantities $X \mid H$, $Y \mid K$, if $X|H \leq Y| K$ when $H \vee K$ is true, then $X|H \leq Y| K$ also when $H \vee K$ is false, so that $X|H \leq Y| K$. This result generalizes Theorem 3, as the symbol $=$ is replaced by $\leq$, and it will be used in Theorem 7 .

Theorem 6 Given any events $H \neq \emptyset, K \neq \emptyset$, and any r.q.'s $X, Y$, let $\Pi$ be the set of the coherent prevision assessments $\mathbb{P}(X \mid H)=\mu, \mathbb{P}(Y \mid K)=v$.

(i) Assume that, for every $(\mu, v) \in \Pi, X|H \leq Y| K$ when $H \vee K$ is true; then $\mu \leq v$ for every $(\mu, v) \in \Pi$.

(ii) For every $(\mu, v) \in \Pi, X|H \leq Y| K$ when $H \vee K$ is true if and only if $X|H \leq Y| K$.

Proof See Appendix A.

The next two results illustrate the monotonicity property of conjunction and disjunction.

Theorem 7 Given $n+1$ arbitrary conditional events $E_{1}\left|H_{1}, \ldots, E_{n+1}\right| H_{n+1}$, with $n \geq 1$, for the conjunctions $\mathcal{C}_{n}$ and $\mathcal{C}_{n+1}$ it holds that $\mathcal{C}_{n+1} \leq \overline{\mathcal{C}}_{n}$.

Proof See Appendix A.

Theorem 8 Given $n+1$ arbitrary conditional events $E_{1}\left|H_{1}, \ldots, E_{n+1}\right| H_{n+1}$, with $n \geq 1$, for the disjunctions $\mathcal{D}_{n}$ and $\mathcal{D}_{n+1}$ it holds that $\mathcal{D}_{n+1} \geq \mathcal{D}_{n}$.

Proof Defining $\mathcal{F}_{n}=\left\{E_{1}\left|H_{1}, \ldots, E_{n}\right| H_{n}\right\}$ and $\mathcal{F}_{n+1}=$ $\mathcal{F}_{n} \cup\left\{E_{n+1} \mid H_{n+1}\right\}$, by Theorems 5 and 7 it holds that

$$
\begin{aligned}
\mathcal{D}_{n+1} & =\mathcal{D}\left(\mathcal{F}_{n+1}\right)=\overline{\mathcal{C}\left(\overline{\mathcal{F}}_{n+1}\right)}=1-\mathcal{C}\left(\overline{\mathcal{F}}_{n+1}\right) \\
& \geq 1-\mathcal{C}\left(\overline{\mathcal{F}}_{n}\right)=\overline{\mathcal{C}\left(\overline{\mathcal{F}}_{n}\right)}=\mathcal{D}_{n} .
\end{aligned}
$$

The next result shows that the conjunction and the disjunction are random quantities with values in the interval $[0,1]$.

Theorem 9 Given $n$ arbitrary conditional events $E_{1} \mid H_{1}$, $\ldots, E_{n} \mid H_{n}$, it holds that: (i) $\mathcal{C}_{n} \in[0,1]$; (ii) $\mathcal{D}_{n} \in[0,1]$.

Proof See Appendix A. 
Remark 6 From Theorem 7, it holds that $\mathcal{C}_{n} \leq \mathcal{C}_{n-1} \leq$ $\ldots \leq \mathcal{C}_{1}$; in particular $\mathbb{P}\left(\mathcal{C}_{n}\right) \leq \mathbb{P}\left(\mathcal{C}_{k}\right), k=1,2, \ldots, n-1$. More generally, for every non empty subset $S$ of $\{1, \ldots, n\}$, it holds that $\mathbb{P}\left(\mathcal{C}_{n}\right) \leq \mathbb{P}\left(\mathcal{C}_{S}\right)$. In particular, $\mathbb{P}\left(\mathcal{C}_{n}\right) \leq$ $P\left(E_{n} \mid H_{n}\right)$. Then, $\mathbb{P}\left(\mathcal{C}_{k}\right) \leq \min \left\{\mathbb{P}\left(\mathcal{C}_{k-1}\right), P\left(E_{k} \mid H_{k}\right)\right\}, k=$ $2,3, \ldots, n$, and by iterating it follows

$\mathbb{P}\left(\mathcal{C}_{n}\right) \leq \min \left\{P\left(E_{1} \mid H_{1}\right), \ldots, P\left(E_{n} \mid H_{n}\right)\right\}$

Likewise, by Theorem 8 ,

$\mathbb{P}\left(\mathcal{D}_{k}\right) \geq \max \left\{\mathbb{P}\left(\mathcal{D}_{k-1}\right),\left(E_{k} \mid H_{k}\right)\right\}, k=2,3, \ldots, n$,

and by iterating it follows

$\mathbb{P}\left(\mathcal{D}_{n}\right) \geq \max \left\{P\left(E_{1} \mid H_{1}\right), \ldots, P\left(E_{n} \mid H_{n}\right)\right\}$.

\section{Coherent assessments on $\left\{\mathcal{C}_{n}, E_{n+1} \mid H_{n+1}, \mathcal{C}_{n+1}\right\}$}

Given any $n+1$ arbitrary conditional events $E_{1} \mid H_{1}, \ldots$, $E_{n+1} \mid H_{n+1}$, let us consider the conjunctions $\mathcal{C}_{n}=$ $\left(E_{1} \mid H_{1}\right) \wedge \cdots \wedge\left(E_{n} \mid H_{n}\right)$ and $\mathcal{C}_{n+1}=\left(E_{1} \mid H_{1}\right) \wedge \cdots \wedge$ $\left(E_{n+1} \mid H_{n+1}\right)$. We set $\mathbb{P}\left(\mathcal{C}_{n}\right)=\mu_{n}, \mathbb{P}\left(\mathcal{C}_{n+1}\right)=\mu_{n+1}$ and $P\left(E_{n+1} \mid H_{n+1}\right)=x_{n+1}$.
Remark 7 Let us consider the points

$Q_{1}=(1,1,1), Q_{2}=(1,0,0), Q_{3}=(0,1,0), Q_{4}=(0,0,0)$.

We observe that the equations of the three planes containing the points $Q_{1}, Q_{2}, Q_{3}$, or $Q_{1}, Q_{2}, Q_{4}$, or $Q_{1}, Q_{3}, Q_{4}$, are $z=x+y-1$, or $z=x$, or $z=y$, respectively. It can be shown that a point $(x, y, z)$ belongs to the convex hull $\mathcal{I}$ of $Q_{1}, Q_{2}, Q_{3}, Q_{4}$ if and only if

$(x, y) \in[0,1]^{2}, \quad \max \{x+y-1,0\} \leq z \leq \min \{x, y\}$.

The convex hull $\mathcal{I}$, which is a tetrahedron with vertices $Q_{1}, Q_{2}, Q_{3}, Q_{4}$, is depicted in Fig. 1 .

We observe that the lower and upper bounds in (14) are the Fréchet-Hoeffding bounds, which characterize the next result.

Theorem 10 Assume that the events $E_{1}, \ldots, E_{n+1}, H_{1}$, $\ldots, H_{n+1}$ are logically independent. Let $\mathcal{I}$ be the convex hull of the points $Q_{1}=(1,1,1), Q_{2}=(1,0,0)$, $Q_{3}=(0,1,0), Q_{4}=(0,0,0)$. Then, the assessment

Fig. 1 Convex hull of the points $Q_{1}, Q_{2}, Q_{3}, Q_{4} \cdot \mathcal{P}^{\prime}=$ $\left(x, y, z^{\prime}\right), \mathcal{P}^{\prime \prime}=\left(x, y, z^{\prime \prime}\right)$, where $(x, y) \in[0,1]^{2}$, $z^{\prime}=\max \{x+y-1,0\}$, $z^{\prime \prime}=\min \{x, y\}$. In the figure the numerical values are: $x=0.6$, $y=0.5, z^{\prime}=0.1$, and $z^{\prime \prime}=0.5$

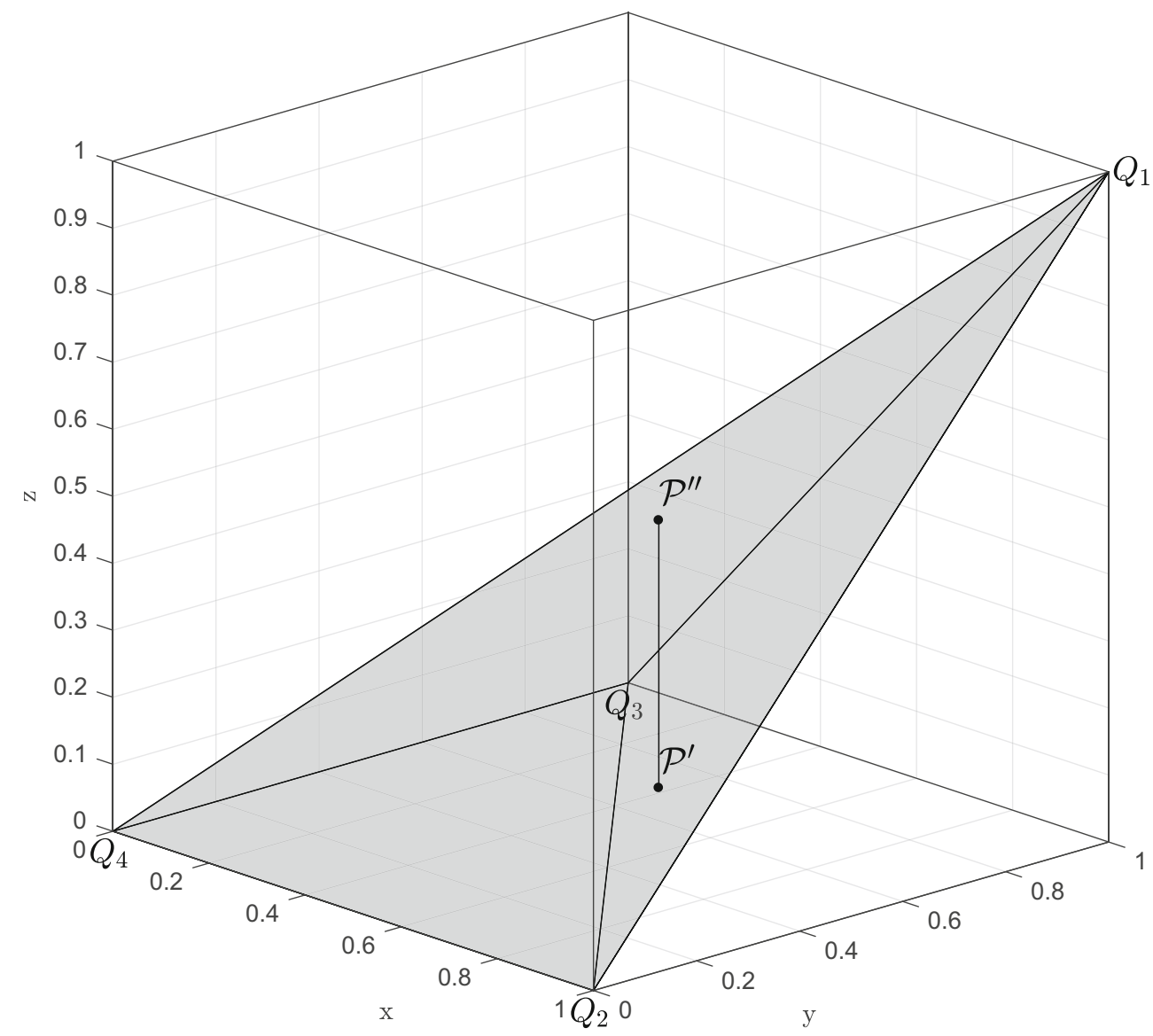


$\mathcal{M}=\left(\mu_{n}, x_{n+1}, \mu_{n+1}\right)$ on the family $\left\{\mathcal{C}_{n}, E_{n+1} \mid H_{n+1}, \mathcal{C}_{n+1}\right\}$ is coherent if and only if $\mathcal{M} \in \mathcal{I}$, that is if and only if

$\left(\mu_{n}, x_{n+1}\right) \in[0,1]^{2}, \quad \mu_{n+1}^{\prime} \leq \mu_{n+1} \leq \mu_{n+1}^{\prime \prime}$,

where $\mu_{n+1}^{\prime}=\max \left\{\mu_{n}+x_{n+1}-1,0\right\}$ and $\mu_{n+1}^{\prime \prime}=$ $\min \left\{\mu_{n}, x_{n+1}\right\}$.

\section{Proof See Appendix A.}

Remark 8 We observe that the representation of each coherent assessment $\mathcal{M}=\left(\mu_{n}, x_{n+1}, \mu_{n+1}\right)$ as a linear convex combination $\lambda_{1} Q_{1}+\lambda_{2} Q_{2}+\lambda_{3} Q_{3}+\lambda_{4} Q_{4}$ (where $\left.\sum_{h=1}^{4} \lambda_{h}=1, \lambda_{h} \geq 0, h=1,2,3,4\right)$ is unique, with

$$
\left\{\begin{array}{l}
\lambda_{1}=\mu_{n+1}=\mathbb{P}\left(\mathcal{C}_{n+1}\right) \geq 0, \\
\lambda_{2}=\mu_{n}-\mu_{n+1}=\mathbb{P}\left(\mathcal{C}_{n}\right)-\mathbb{P}\left(\mathcal{C}_{n+1}\right) \geq 0, \\
\lambda_{3}=x_{n+1}-\mu_{n+1}=P\left(E_{n+1} \mid H_{n+1}\right)-\mathbb{P}\left(\mathcal{C}_{n+1}\right) \geq 0, \\
\lambda_{4}=1-\mu_{n}-x_{n+1}+\mu_{n+1} \\
\quad=1-\mathbb{P}\left(\mathcal{C}_{n}\right)-P\left(E_{n+1} \mid H_{n+1}\right)+\mathbb{P}\left(\mathcal{C}_{n+1}\right) \geq 0 .
\end{array}\right.
$$

In particular, concerning the extreme cases $\mu_{n+1}=$ $\mu_{n+1}^{\prime}$, or $\mu_{n+1}=\mu_{n+1}^{\prime \prime}$, we can examine four cases: 1 ) $\mu_{n+1}^{\prime}=\mu_{n}+x_{n+1}-1>0$;2) $\mu_{n+1}^{\prime}=0$; 3) $\mu_{n+1}^{\prime \prime}=\mu_{n}$ and 4) $\mu_{n+1}^{\prime \prime}=x_{n+1}$.

In the case 1 the point $\mathcal{M}=\left(\mu_{n}, x_{n+1}, \mu_{n+1}\right)$ is a linear convex combination $\lambda_{1} Q_{1}+\lambda_{2} Q_{2}+\lambda_{3} Q_{3}+\lambda_{4} Q_{4}$, with $\lambda_{1}=\mu_{n+1}^{\prime}=\mu_{n}+x_{n+1}-1, \lambda_{2}=1-x_{n+1}, \lambda_{3}=$ $1-\mu_{n}, \lambda_{4}=0$.

In the case 2 it holds that $\lambda_{1}=\mu_{n+1}^{\prime}=0, \lambda_{2}=\mu_{n}, \lambda_{3}=$ $x_{n+1}, \lambda_{4}=1-\mu_{n}+x_{n+1}$.

In the case 3 it holds that $\lambda_{1}=\mu_{n+1}^{\prime \prime}=\mu_{n}, \lambda_{2}=$ $0, \lambda_{3}=x_{n+1}-\mu_{n}, \lambda_{4}=1-x_{n+1}$.

In the case 4 it holds that $\lambda_{1}=\mu_{n+1}^{\prime \prime}=x_{n+1}, \lambda_{2}=$ $\mu_{n}-x_{n+1}, \lambda_{3}=0, \lambda_{4}=1-\mu_{n}$.

\section{Probabilistic inference from $\mathcal{C}_{n+1}$ to $\left\{\mathcal{C}_{n}, E_{n+1} \mid H_{n+1}\right\}$}

In this section, given any coherent prevision assessment $\mu_{n+1}$ on $\mathcal{C}_{n+1}$, we find the set of coherent extensions $\left(\mu_{n}, x_{n+1}\right)$ on $\left\{\mathcal{C}_{n}, E_{n+1} \mid H_{n+1}\right\}$. As we will see, it is enough to illustrate the case $n=1$, by finding the set of coherent extensions $(x, y)$ on $\left\{E_{1}\left|H_{1}, E_{2}\right| H_{2}\right\}$ of any assessment $z=$ $\mathbb{P}\left[\left(E_{1} \mid H_{1}\right) \wedge\left(E_{2} \mid H_{2}\right)\right] \in[0,1]$.

Theorem 11 Given any prevision assessment $z$ on $\left(E_{1} \mid H_{1}\right) \wedge\left(E_{2} \mid H_{2}\right)$, with $z \in[0,1]$, with $E_{1}, H_{1}, E_{2}, H_{2}$ logically independent, with $H_{1} \neq \emptyset$ and $H_{2} \neq \emptyset$, the extension $x=P\left(E_{1} \mid H_{1}\right), y=P\left(E_{2} \mid H_{2}\right)$ is coherent if and only if $(x, y)$ belongs to the set $T_{z}=\{(x, y): x \in[z, 1], y \in$ $[z, 1+z-x]\}$.

Proof We recall that, by logical independence of $E_{1}, H_{1}$, $E_{2}, H_{2}$, the assessment $(x, y)$ is coherent for every $(x, y) \in[0,1]^{2}$. From Theorem 4, the set $\Pi$ of all coherent assessment $(x, y, z)$ on $\left\{E_{1}\left|H_{1}, E_{2}\right| H_{2},\left(E_{1} \mid H_{1}\right) \wedge\right.$ $\left.\left(E_{2} \mid H_{2}\right)\right\}$ is $\Pi=\left\{(x, y, z):(x, y) \in[0,1]^{2}, \max \{x+y-\right.$ $1,0\} \leq z \leq \min \{x, y\}\}$. We note that

$$
\begin{aligned}
\Pi & =\{(x, y, z): z \in[0,1], x \in[z, 1], y \in[z, z+1-x]\}= \\
& =\left\{(x, y, z): z \in[0,1],(x, y) \in T_{z}\right\} .
\end{aligned}
$$

Then, $(x, y)$ is a coherent extension of $z$ if and only if $(x, y) \in T_{z}$.

Remark 9 We observe that, given any $z \in[0,1]$ and defining $\Pi_{z}=\left\{(x, y, z):(x, y) \in T_{z}\right\}$, it holds that $\Pi=\bigcup_{z \in[0,1]} \Pi_{z}$ (see Fig. 2). The set $\Pi$ is the tetrahedron depicted in Fig. 1. Hence, contrarily to the general case, for the family $\left\{E_{1}\left|H_{1}, E_{2}\right| H_{2},\left(E_{1} \mid H_{1}\right) \wedge\left(E_{2} \mid H_{2}\right)\right\}$ the set of coherent prevision assessments $\Pi$ is convex. Indeed, $\Pi$ is also the (convex) set of coherent probability assessment $(x, y, z)$ on the family of unconditional events $\left\{E_{1}, E_{2}, E_{1} E_{2}\right\}$. We recall that, assuming $H_{1} \wedge H_{2}=$ $\emptyset$, the set of coherent prevision assessments $(x, y, z)$ on $\left\{E_{1}\left|H_{1}, E_{2}\right| H_{2},\left(E_{1} \mid H_{1}\right) \wedge\left(E_{2} \mid H_{2}\right)\right\}$ is the surface $\left\{(x, y, z):(x, y) \in[0,1]^{2}, z=x y\right\}$, which is a strict non-convex subset of $\Pi$ (see [23, Section 5]).

Theorem 12 Given any prevision assessment $\mu_{n+1}=$ $\mathbb{P}\left(\mathcal{C}_{n+1}\right) \in[0,1]$, with $\mu_{n+1} \in[0,1]$, the extension $\mu_{n}=\mathbb{P}\left(\mathcal{C}_{n}\right), x_{n+1}=P\left(E_{n+1} \mid H_{n+1}\right)$ is coherent if and only if

$$
\begin{aligned}
\left(\mu_{n}, x_{n+1}\right) \in & \left\{\left(\mu_{n}, x_{n+1}\right): \mu_{n} \in\left[\mu_{n+1}, 1\right],\right. \\
& \left.x_{n+1} \in\left[\mu_{n+1}, 1+\mu_{n+1}-\mu_{n}\right]\right\} .
\end{aligned}
$$

Proof From Theorem 10, the set $\Pi$ of all coherent assessment $\left(\mu_{n}, x_{n+1}, \mu_{n+1}\right)$ on $\left\{\mathcal{C}_{n}, E_{n+1} \mid H_{n+1}, \mathcal{C}_{n+1}\right\}$ is $\Pi=\left\{\left(\mu_{n}, x_{n+1}, \mu_{n+1}\right):\left(\mu_{n}, x_{n+1}\right) \in[0,1]^{2}, \max \left\{\mu_{n}+\right.\right.$ $\left.\left.x_{n+1}-1,0\right\} \leq \mu_{n+1} \leq \min \left\{\mu_{n}, x_{n+1}\right\}\right\}$. Moreover, as observed in the proof of Theorem 11, the set $\Pi$ coincides with the set

$\left\{\left(\mu_{n}, x_{n+1}, \mu_{n+1}\right): \mu_{n+1} \in[0,1], \mu_{n} \in\left[\mu_{n+1}, 1\right]\right.$,

$$
\left.x_{n+1} \in\left[\mu_{n+1}, 1+\mu_{n+1}-\mu_{n}\right]\right\} \text {. }
$$

Then, $\left(\mu_{n}, x_{n+1}\right)$ is a coherent extension of $\mu_{n+1}$ if and only if $\left(\mu_{n}, x_{n+1}\right)$ belongs to the set $\left\{\left(\mu_{n}, x_{n+1}\right): \mu_{n} \in\right.$ $\left.\left[\mu_{n+1}, 1\right], x_{n+1} \in\left[\mu_{n+1}, 1+\mu_{n+1}-\mu_{n}\right]\right\}$. 
Fig. 2 Set $\Pi$ of all coherent assessments $(x, y, z)$ on $\left\{E_{1}\left|H_{1}, E_{2}\right| H_{2},\left(E_{1} \mid H_{1}\right) \wedge\right.$ $\left.\left(E_{2} \mid H_{2}\right)\right\}$. Notice that $\Pi=\bigcup_{z \in[0,1]} \Pi_{z}$, where for each given $z \in[0,1]$ the set $\Pi_{z}$ is the triangle

$\left\{(x, y, z):(x, y) \in T_{z}\right\}$, with

$T_{z}=\{(x, y): x \in[z, 1], y \in$ $[z, 1+z-x]\}$

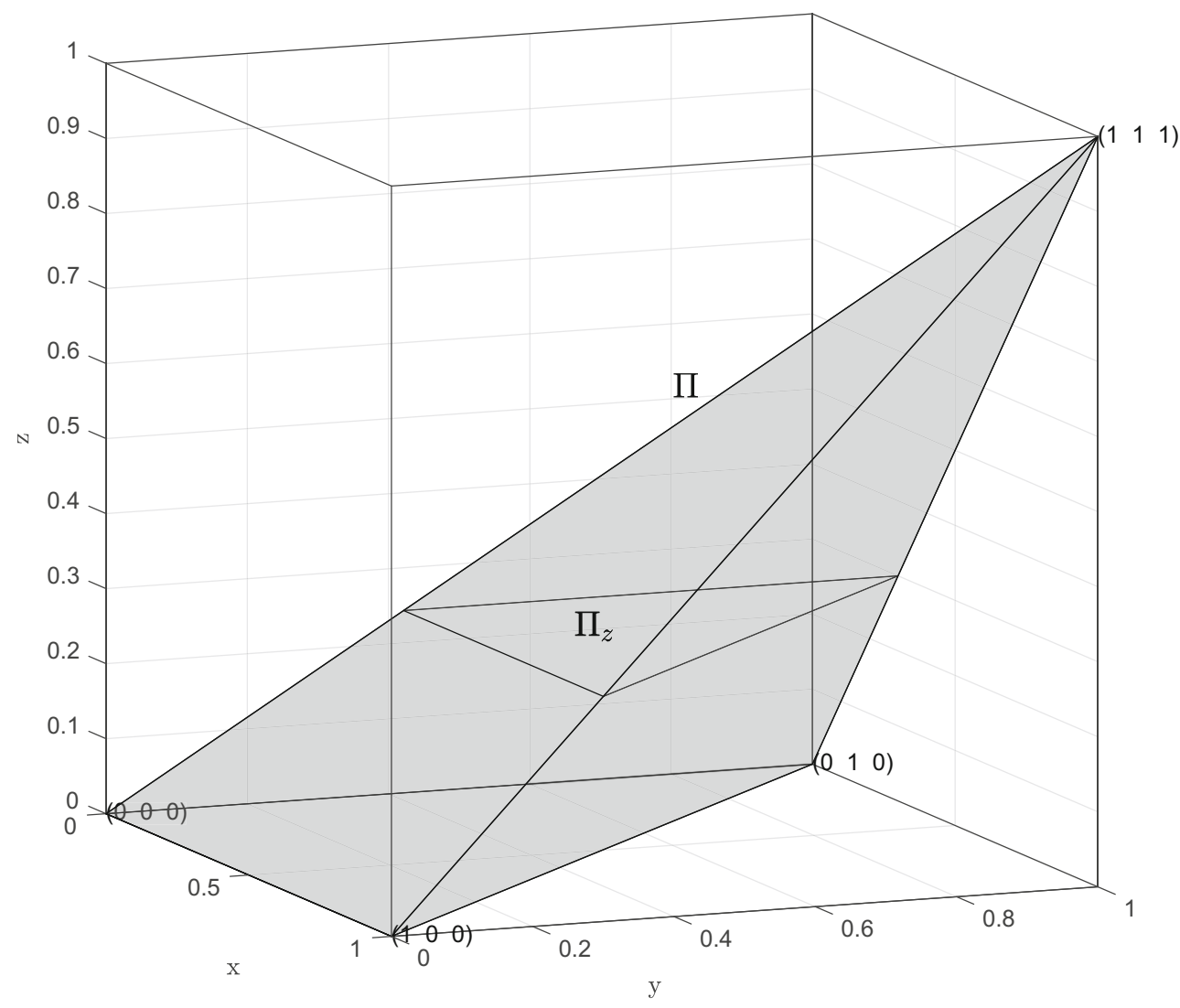

\section{Fréchet-Hoeffding bounds}

In the next result we show that the prevision of the conjunction $\mathcal{C}_{n}=E_{1}\left|H_{1} \wedge \cdots \wedge E_{n}\right| H_{n}$ satisfies the Fréchet-Hoeffding bounds.

Theorem 13 Let be given $n$ conditional events $E_{1} \mid H_{1}$, $E_{2}\left|H_{2}, \ldots, E_{n}\right| H_{n}$, with $x_{i}=P\left(E_{i} \mid H_{i}\right), i=1,2, \ldots, n$, and with $\mathbb{P}\left(\mathcal{C}_{n}\right)=\mu_{n}$. Then

$\max \left\{x_{1}+\cdots+x_{n}-(n-1), 0\right\} \leq \mu_{n} \leq \min \left\{x_{1}, \ldots, x_{n}\right\}$.

Proof From Theorem 10, it holds that

$$
\begin{aligned}
\mu_{n} \geq & \mu_{n-1}+x_{n}-1 \geq \mu_{n-2}+x_{n-1}+x_{n}-2 \geq \cdots \geq \\
& x_{1}+\cdots+x_{n}-(n-1) .
\end{aligned}
$$

Then, by inequality (12) and by Theorem 9 it holds that the inequalities in (15) are satisfied.

Likewise, the following result holds for the prevision $\eta_{n}$ of the disjunction $\mathcal{D}_{n}=E_{1}\left|H_{1} \vee E_{2}\right| H_{2} \vee \cdots \vee E_{n} \mid H_{n}$.
Theorem 14 Let be given $n$ conditional events $E_{1} \mid H_{1}$, $E_{2}\left|H_{2}, \ldots, E_{n}\right| H_{n}$, with $x_{i}=P\left(E_{i} \mid H_{i}\right), i=1,2, \ldots, n$, and with $\mathbb{P}\left(\mathcal{D}_{n}\right)=\eta_{n}$. Then

$\max \left\{x_{1}, \ldots, x_{n}\right\} \leq \eta_{n} \leq \min \left\{x_{1}+\cdots+x_{n}, 1\right\}$.

Proof By Definition 9, Theorems 5 and 13, defining $\overline{\mathcal{F}}_{n}=$ $\left\{\bar{E}_{1}\left|H_{1}, \bar{E}_{2}\right| H_{2}, \ldots, \bar{E}_{n} \mid H_{n}\right\}$ it holds that

$$
\begin{aligned}
\mathbb{P}\left(\mathcal{D}_{n}\right) & =1-\mathbb{P}\left(\overline{\mathcal{D}}_{n}\right)=1-\mathbb{P}\left(\mathcal{C}\left(\overline{\mathcal{F}}_{n}\right)\right)=1-\mathbb{P}\left(\bigwedge_{i=1}^{n}\left(\overline{E_{i}} \mid H_{i}\right)\right) \\
& \leq 1-\left[\left(1-x_{1}\right)+\cdots+\left(1-x_{n}\right)-(n-1)\right] \\
& =x_{1}+\cdots+x_{n} .
\end{aligned}
$$

Then, by (13) and by Theorem 9, the inequalities in (16) are satisfied.

\section{Conjunction of three conditional events}

Given a family of three conditional events $\mathcal{F}=$ $\left\{E_{1}\left|H_{1}, E_{2}\right| H_{2}, E_{3} \mid H_{3}\right\}$, we set $P\left(E_{i} \mid H_{i}\right)=x_{i}, i=1,2,3$, 
$\mathbb{P}\left[\left(E_{i} \mid H_{i}\right) \wedge\left(E_{j} \mid H_{j}\right)\right]=x_{i j}=x_{j i}, i \neq j$, and $x_{123}=$ $\mathbb{P}\left[\left(E_{1} \mid H_{1}\right) \wedge\left(E_{2} \mid H_{2}\right) \wedge\left(E_{3} \mid H_{3}\right)\right]$. Then, by Definition 5, the conjunction of $E_{1}\left|H_{1}, E_{2}\right| H_{2}, E_{3} \mid H_{3}$ is the conditional random quantity

$$
\begin{aligned}
\mathcal{C}(\mathcal{F})= & \left(E_{1} \mid H_{1}\right) \wedge\left(E_{2} \mid H_{2}\right) \wedge\left(E_{3} \mid H_{3}\right) \\
= & \begin{cases}1, & \text { if } E_{1} H_{1} E_{2} H_{2} E_{3} H_{3} \text { is true, } \\
0, & \text { if } \bar{E}_{1} H_{1} \vee \bar{E}_{2} H_{2} \vee \bar{E}_{3} H_{3} \text { is true, } \\
x_{1}, & \text { if } \bar{H}_{1} E_{2} H_{2} E_{3} H_{3} \text { is true, } \\
x_{2}, & \text { if } \bar{H}_{2} E_{1} H_{1} E_{3} H_{3} \text { is true, } \\
x_{3}, & \text { if } \bar{H}_{3} E_{1} H_{1} E_{2} H_{2} \text { is true, } \\
x_{12}, & \text { if } \bar{H}_{1} \bar{H}_{2} E_{3} H_{3} \text { is true, } \\
x_{13}, & \text { if } \bar{H}_{1} \bar{H}_{3} E_{2} H_{2} \text { is true, } \\
x_{23}, & \text { if } \bar{H}_{2} \bar{H}_{3} E_{1} H_{1} \text { is true, } \\
x_{123}, & \text { if } \bar{H}_{1} \bar{H}_{2} \bar{H}_{3} \text { is true. }\end{cases}
\end{aligned}
$$

Remark 10 Notice that in the betting scheme $x_{123}$ is the quantity to be paid in order to receive $\mathcal{C}(\mathcal{F})$. Assuming that the assessment $\left(x_{1}, x_{2}, x_{3}, x_{12}, x_{13}, x_{23}\right)$ on $\left\{E_{1} \mid H_{1}\right.$, $E_{2}\left|H_{2}, E_{3}\right| H_{3},\left(E_{1} \mid H_{1}\right) \wedge\left(E_{2} \mid H_{2}\right),\left(E_{1} \mid H_{1}\right) \wedge\left(E_{3} \mid H_{3}\right)$, $\left.\left(E_{2} \mid H_{2}\right) \wedge\left(E_{3} \mid H_{3}\right)\right\}$ is coherent, we are interested in finding the values $x_{123}$ which are a coherent extension of $\left(x_{1}, x_{2}, x_{3}, x_{12}, x_{13}, x_{23}\right)$. Of course, as $x_{i} \in[0,1], i=$ $1,2,3$, and $x_{i j} \in[0,1], i \neq j$, a necessary condition for coherence is $x_{123} \in[0,1]$.

From Remark 5 and Proposition 1 the conjunction $\mathcal{C}(\mathcal{F})$ is invariant with respect to any given permutation $\left(i_{1}, i_{2}, i_{3}\right)$ of $(1,2,3)$; that is $\left.\mathcal{C}(\mathcal{F})=\left(E_{i_{1}} \mid H_{i_{1}}\right) \wedge\left(E_{i_{2}} \mid H_{i_{2}}\right)\right) \wedge\left(E_{i_{3}} \mid H_{i_{3}}\right)$, for any permutation $\left(i_{1}, i_{2}, i_{3}\right)$ of $(1,2,3)$.

\subsection{Study of coherence}

Notice that in general, if we assess the values $x_{S}=\mathbb{P}\left(\mathcal{C}_{S}\right)$ for some $S \subset\{1,2 \ldots, n\}$, then the study of coherence may be very complex. In this section we study coherence in the case $n=3$ when we assess the prevision $x_{S}=\mathbb{P}\left(\mathcal{C}_{S}\right)$ for every $S \subseteq\{1,2,3\}$. In the next result we determine the set of coherent assessments $\mathcal{M}=\left(x_{1}, x_{2}, x_{3}, x_{12}, x_{13}, x_{23}\right.$, $\left.x_{123}\right)$ on the family $\mathcal{F}=\left\{E_{1}\left|H_{1}, E_{2}\right| H_{2}, E_{3} \mid H_{3},\left(E_{1} \mid H_{1}\right) \wedge\right.$ $\left(E_{2} \mid H_{2}\right), \quad\left(E_{1} \mid H_{1}\right) \wedge\left(E_{3} \mid H_{3}\right),\left(E_{2} \mid H_{2}\right) \wedge\left(E_{3} \mid H_{3}\right)$, $\left.\left(E_{1} \mid H_{1}\right) \wedge\left(E_{2} \mid H_{2}\right) \wedge\left(E_{3} \mid H_{3}\right)\right\}=\left\{\mathcal{C}_{S}: \emptyset \neq S \subseteq\right.$ $\{1,2,3\}\}$.

Theorem 15 Assume that the events $E_{1}, E_{2}, E_{3}, H_{1}, H_{2}, H_{3}$ are logically independent, with $H_{1} \neq \emptyset, H_{2} \neq \emptyset, H_{3} \neq$ $\emptyset$. Then, the set $\Pi$ of all coherent assessments $\mathcal{M}=$ $\left(x_{1}, x_{2}, x_{3}, x_{12}, x_{13}, x_{23}, x_{123}\right)$ on $\mathcal{F}=\left\{E_{1}\left|H_{1}, E_{2}\right| H_{2}, E_{3} \mid\right.$ $H_{3},\left(E_{1} \mid H_{1}\right) \mid \wedge\left(E_{2} \mid H_{2}\right),\left(E_{1} \mid H_{1}\right) \wedge\left(E_{3} \mid H_{3}\right),\left(E_{2} \mid H_{2}\right) \wedge$ $\left.\left(E_{3} \mid H_{3}\right), \quad\left(E_{1} \mid H_{1}\right) \wedge\left(E_{2} \mid H_{2}\right) \wedge\left(E_{3} \mid H_{3}\right)\right\}$ is the set of points $\left(x_{1}, x_{2}, x_{3}, x_{12}, x_{13}, x_{23}, x_{123}\right)$ which satisfy the following conditions

$\left(x_{1}, x_{2}, x_{3}\right) \in[0,1]^{3}$,

$\max \left\{x_{1}+x_{2}-1, x_{13}+x_{23}-x_{3}, 0\right\} \leq x_{12} \leq \min \left\{x_{1}, x_{2}\right\}$,

$\max \left\{x_{1}+x_{3}-1, x_{12}+x_{23}-x_{2}, 0\right\} \leq x_{13} \leq \min \left\{x_{1}, x_{3}\right\}$,

$\left\{\max \left\{x_{2}+x_{3}-1, x_{12}+x_{13}-x_{1}, 0\right\} \leq x_{23} \leq \min \left\{x_{2}, x_{3}\right\}\right.$,

$1-x_{1}-x_{2}-x_{3}+x_{12}+x_{13}+x_{23} \geq 0$,

$x_{123} \geq \max \left\{0, x_{12}+x_{13}-x_{1}, x_{12}+x_{23}-x_{2}, x_{13}+x_{23}-x_{3}\right\}$,

$x_{123} \leq \min \left\{x_{12}, x_{13}, x_{23}, 1-x_{1}-x_{2}-x_{3}+x_{12}+x_{13}+x_{23}\right\}$.

Proof See Appendix A.

We observe that, from (18) it follows that the coherence of $\left(x_{1}, x_{2}, x_{3}, x_{12}, x_{13}, x_{23}\right)$ amounts to the inequality

$$
\begin{aligned}
& \min \left\{x_{12}, x_{13}, x_{23}, 1-x_{1}-x_{2}-x_{3}+x_{12}+x_{13}+x_{23}\right\} \\
& \leq \max \left\{0, x_{12}+x_{13}-x_{1}, x_{12}+x_{23}-x_{2}, x_{13}+x_{23}-x_{3}\right\} .
\end{aligned}
$$

Then, by Theorem 15 it follows

Corollary 1 For any coherent assessment $\left(x_{1}, x_{2}, x_{3}, x_{12}\right.$, $\left.x_{13}, x_{23}\right)$ on

$\left\{E_{1}\left|H_{1}, E_{2}\right| H_{2}, E_{3} \mid H_{3},\left(E_{1} \mid H_{1}\right) \wedge\left(E_{2} \mid H_{2}\right)\right.$,

$\left.\left(E_{1} \mid H_{1}\right) \wedge\left(E_{3} \mid H_{3}\right),\left(E_{2} \mid H_{2}\right) \wedge\left(E_{3} \mid H_{3}\right)\right\}$

the extension $x_{123}$ on $\left(E_{1} \mid H_{1}\right) \wedge\left(E_{2} \mid H_{2}\right) \wedge\left(E_{3} \mid H_{3}\right)$ is coherent if and only if $x_{123} \in\left[x_{123}^{\prime}, x_{123}^{\prime \prime}\right]$, where

$$
\begin{aligned}
x_{123}^{\prime}=\max & \left\{0, x_{12}+x_{13}-x_{1}, x_{12}+x_{23}-x_{2}, x_{13},\right. \\
& \left.+x_{23}-x_{3}\right\} \\
x_{123}^{\prime \prime}=\min & \left\{x_{12}, x_{13}, x_{23}, 1-x_{1}-x_{2}-x_{3}+x_{12}\right. \\
& \left.+x_{13}+x_{23}\right\} .
\end{aligned}
$$

Proof As shown in (18), (see also (46) in the Appendix A), the coherence of $\left(x_{1}, x_{2}, x_{3}, x_{12}, x_{13}, x_{23}, x_{123}\right)$ amounts to the condition

$$
\begin{gathered}
\min \left\{x_{12}, x_{13}, x_{23}, 1-x_{1}-x_{2}-x_{3}+x_{12}+x_{13}+x_{23}\right\} \leq x_{123} \\
\leq \max \left\{0, x_{12}+x_{13}-x_{1}, x_{12}+x_{23}-x_{2}, x_{13}+x_{23}-x_{3}\right\}
\end{gathered}
$$

Then, in particular, the extension $x_{123}$ on $\left(E_{1} \mid H_{1}\right) \wedge$ $\left(E_{2} \mid H_{2}\right) \wedge\left(E_{3} \mid H_{3}\right)$ is coherent if and only if $x_{123} \in$ $\left[x_{123}^{\prime}, x_{123}^{\prime \prime}\right]$, where

$$
\begin{gathered}
x_{123}^{\prime}=\max \left\{0, x_{12}+x_{13}-x_{1}, x_{12}+x_{23}-x_{2},\right. \\
\left.x_{13}+x_{23}-x_{3}\right\}, \\
x_{123}^{\prime \prime}=\min \left\{x_{12}, x_{13}, x_{23}, 1-x_{1}-x_{2}-x_{3}+x_{12}\right. \\
\left.+x_{13}+x_{23}\right\} .
\end{gathered}
$$




\subsection{The Case $H_{1}=H_{2}=H_{3}$}

We recall that in case of logical dependencies, the set of all coherent assessments may be smaller than that one associated with the case of logical independence. However, in this section we show that the results of Theorem 15 and Corollary 1 still hold when the conditioning events $H_{1}, H_{2}$, and $H_{3}$ coincide.

Theorem 16 Let be given any logically independent events $E_{1}, E_{2}, E_{3}, H$, with $H \neq \emptyset$. Then, the set $\Pi$ of all coherent assessments $\mathcal{M}=\left(x_{1}, x_{2}, x_{3}, x_{12}, x_{13}, x_{23}, x_{123}\right)$ on $\mathcal{F}=\left\{E_{1}\left|H, E_{2}\right| H, E_{3} \mid H,\left(E_{1} \mid H\right) \wedge\left(E_{2} \mid H\right),\left(E_{1} \mid H\right) \wedge\right.$ $\left.\left(E_{3} \mid H\right),\left(E_{2} \mid H\right) \wedge\left(E_{3} \mid H\right),\left(E_{1} \mid H\right) \wedge\left(E_{2} \mid H\right) \wedge\left(E_{3} \mid H\right)\right\}$ is the set of points $\left(x_{1}, x_{2}, x_{3}, x_{12}, x_{13}, x_{23}, x_{123}\right)$ which satisfy the conditions in formula (18).

Proof See Appendix A.

Corollary 2 For any coherent assessment $\left(x_{1}, x_{2}, x_{3}, x_{12}\right.$, $\left.x_{13}, x_{23}\right)$ on

$\left\{E_{1}\left|H, E_{2}\right| H, E_{3}\left|H,\left(E_{1} E_{2}\right)\right| H,\left(E_{1} E_{3}\right)\left|H,\left(E_{2} E_{3}\right)\right| H\right\}$

the extension $x_{123}$ on $\left(E_{1} E_{2} E_{3}\right) \mid H$ is coherent if and only if $x_{123} \in\left[x_{123}^{\prime}, x_{123}^{\prime \prime}\right]$, where $x_{123}^{\prime}$ and $x_{123}^{\prime \prime}$ are defined in (19).

Proof The proof is the same as for Corollary 1.

Of course, the results of Theorem 16 and Corollary 2 still hold in the unconditional case where $H=\Omega$.

Remark 11 As shown in this section, a consistent management of conjunctions (and/or disjunctions) defined on a given family of conditional events $\mathcal{F}$ essentially requires an (iterative) coherence checking and propagation of probability and prevision assessments on compounded conditionals, for each subfamily of $\mathcal{F}$. Then, an analysis of complexity in our context would be of the same kind of the exhaustive complexity analysis given in [5] for probabilistic reasoning under coherence.

\section{Characterization of p-consistency and p-entailment with applications to nonmonotonic reasoning}

In this section we apply our notion of conjunction to characterize the notions of p-consistency and p-entailment. Then, we examine some inference rules related with probabilistic nonmonotonic reasoning. We also briefly describe a characterization of p-entailment by a notion of iterated conditioning, in the case of two premises. We recall below the notions of p-consistency and p-entailment of Adams [2] as formulated for conditional events in the setting of coherence (see, e.g., [5, 20, 24]).

Definition 10 Let $\mathcal{F}=\left\{E_{i} \mid H_{i}, \quad i=1, \ldots, n\right\}$ be a family of $n$ conditional events. Then, $\mathcal{F}$ is $p$-consistent if and only if the probability assessment $\left(p_{1}, p_{2}, \ldots, p_{n}\right)=$ $(1,1, \ldots, 1)$ on $\mathcal{F}$ is coherent.

Definition 11 A p-consistent family $\mathcal{F}=\left\{E_{i} \mid H_{i}, \quad i=\right.$ $1, \ldots, n\}$ p-entails a conditional event $E_{n+1} \mid H_{n+1}$ if and only if for any coherent probability assessment $\left(p_{1}, \ldots, p_{n}, p_{n+1}\right)$ on $\mathcal{F} \cup\left\{E_{n+1} \mid H_{n+1}\right\}$ it holds that: if $p_{1}=\cdots=p_{n}=1$, then $p_{n+1}=1$.

We recall below the notion of logical implication [31] between two conditional events.

Definition 12 Given two conditional events $A \mid H$ and $B \mid K$ we say that $A \mid H$ logically implies $B \mid K$, which we denote by $A|H \subseteq B| K$, if and only if $A H$ true implies $B K$ true and $\bar{B} K$ true implies $\bar{A} H$ true; that is: $A H \subseteq B K$ and $\bar{B} K \subseteq \bar{A} H$.

A suitable conditional event, named "implication", which could be related with the relation of logical implication was introduced by de Finetti in [16]. We observe that, by coherence, it holds that (see, e.g., [25, Theorem 7]).

$A|H \subseteq B| K \quad \Longrightarrow \quad P(A \mid H) \leq P(B \mid K)$.

We also recall the notion of quasi conjunction for a general family of $n$ conditional events.

Definition 13 Given a family $\mathcal{F}=\left\{E_{i} \mid H_{i}, i=1, \ldots, n\right\}$ of $n$ conditional events, the quasi conjunction $Q C(\mathcal{F})$ of the conditional events in $\mathcal{F}$ is defined as the following conditional event

$Q C(\mathcal{F})=\bigwedge_{i=1}^{n}\left(\bar{H}_{i} \vee E_{i} H_{i}\right) \mid\left(\bigvee_{i=1}^{n} H_{i}\right)$.

Remark 12 We observe that, by Definition 13, based on (9) the quasi conjunction can be represented as

$Q C(\mathcal{F})=\sum_{h=0}^{m} v_{h} C_{h}$,

where $v_{h}=\left\{\begin{array}{l}1, \text { if } S_{h}^{\prime} \neq \emptyset \text { and } S_{h}^{\prime \prime}=\emptyset, \\ 0, \text { if } S_{h}^{\prime \prime} \neq \emptyset \\ v, \text { if } S_{h}^{\prime \prime \prime}=\{1, \ldots, n\},\end{array}\right.$ 
where $v=P(Q C(\mathcal{F}))$. Therefore, by (10), (21), and by also recalling Theorem 6 , it holds that $z_{h} \leq v_{h}, h=$ $0,1, \ldots, m$; thus

$\mathcal{C}(\mathcal{F}) \leq Q C(\mathcal{F})$.

In particular, if $\mathcal{F}$ is p-consistent and $P\left(E_{i} \mid H_{i}\right)=1, i=$ $1, \ldots, n$, then from $(15)$ it holds that $x_{S}=\mathbb{P}\left(\mathcal{C}\left(\mathcal{F}_{S}\right)\right)=1$ for every $S \subseteq\{1,2, \ldots, n\}$, where $\mathcal{F}_{S}=\left\{E_{i} \mid H_{i} \in \mathcal{F}: i \in\right.$ $S\}$; then $z_{h}=v_{h}, h=0,1, \ldots, m$, and $\mathcal{C}(\mathcal{F})=Q C(\mathcal{F})$.

\subsection{Characterization of $p$-consistency and p-entailment}

We illustrate below a characterization of p-consistency of a family $\mathcal{F}$ in terms of the coherence of the prevision assessment $\mathbb{P}[\mathcal{C}(\mathcal{F})]=1$.

Theorem 17 A family of $n$ conditional events $\mathcal{F}=$ $\left\{E_{1}\left|H_{1}, \ldots, E_{n}\right| H_{n}\right\}$ is p-consistent if and only if the prevision assessment $\mathbb{P}[\mathcal{C}(\mathcal{F})]=1$ is coherent.

Proof $(\Rightarrow)$ By Definition 10 , as $\mathcal{F}$ is p-consistent, the probability assessment $\left(x_{1}, x_{2}, \ldots, x_{n}\right)=(1,1, \ldots, 1)$ on $\mathcal{F}$ is coherent. Then, by (15) the extension $\mathbb{P}[\mathcal{C}(\mathcal{F})]=1$ is unique and of course coherent.

$(\Leftarrow)$ By $(15)$ it holds that $\mathbb{P}[\mathcal{C}(\mathcal{F})] \leq \min \left\{x_{1}, \ldots, x_{n}\right\}$ and hence $\mathbb{P}[\mathcal{C}(\mathcal{F})]=1$ implies $\left(x_{1}, x_{2}, \ldots, x_{n}\right)=$ $(1,1, \ldots, 1)$ on $\mathcal{F}$. Moreover, the coherence of $\mathbb{P}[\mathcal{C}(\mathcal{F})]=$ 1 requires that the (unique) extension $(1,1, \ldots, 1)$ on $\mathcal{F}$ be coherent. Thus, $\mathcal{F}$ is p-consistent.

We observe that, in the case where $H_{1}=\ldots=$ $H_{n}=H$, the assessment $P\left(E_{1} \mid H\right)=\ldots P\left(E_{n} \mid H\right)=$ 1 is coherent (that is, $\mathcal{F}$ is p-consistent) if and only if $P\left[\left(E_{1} \cdots E_{n}\right) \mid H\right]=1$ is coherent.

The next theorem gives a characterization of p-entailment in terms of a result which involves suitable conjunctions associated with the premise set and the conclusion of the given inference rule.

Theorem 18 Let be given a p-consistent family of $n$ conditional events $\mathcal{F}=\left\{E_{1}\left|H_{1}, \ldots, E_{n}\right| H_{n}\right\}$ and $a$ further conditional event $E_{n+1} \mid H_{n+1}$. Then, the following assertions are equivalent:

(i) $\mathcal{F}$ p-entails $E_{n+1} \mid H_{n+1}$;

(ii) the conjunction $\mathcal{C}_{n+1}=\left(E_{1} \mid H_{1}\right) \wedge \cdots \wedge\left(E_{n} \mid H_{n}\right) \wedge$ $\left(E_{n+1} \mid H_{n+1}\right)$ coincides with the conjunction $\mathcal{C}_{n}=$ $\left(E_{1} \mid H_{1}\right) \wedge \cdots \wedge\left(E_{n} \mid H_{n}\right)$

(iii) the inequality $\mathcal{C}_{n} \leq\left(E_{n+1} \mid H_{n+1}\right)$ is satisfied.

Proof See Appendix A.
As a first simple application of Theorem 18 we observe that, given two conditional events $A \mid H$, with $A H \neq \emptyset$, and $B \mid K$, the p-entailment of $B \mid K$ from $A \mid H$ amounts to the condition (ii), i.e., $A|H \wedge B| K=A \mid H$, or equivalently condition (iii), i.e., $A|H \leq B| K$. In particular, (ii) and (iii) are both satisfied when $A|H \subseteq B| K$.

\subsection{Applications to some $p$-valid inference rules}

We recall that an inference from a p-consistent family $\mathcal{F}$ to $E \mid H$ is $p$-valid if and only if $\mathcal{F}$ p-entails $E \mid H$. We will examine some p-valid inference rules by verifying that conditions (ii) and (iii) in Theorem 18 are satisfied. In particular we consider the following inference rules of System P: And, Cut, CM, and Or. In what follows, if not specified otherwise, the basic events are assumed to be logically independent.

And rule The family $\{B|A, C| A\}$ p-entails $B C \mid A$. It holds that $(B \mid A) \wedge(C \mid A)=B C \mid A=(B \mid A) \wedge(C \mid A) \wedge(B C \mid A)$ and $(B \mid A) \wedge(C \mid A)=B C|A \leq B C| A$; that is, conditions (ii) and (iii) are satisfied.

Cut rule The family $\{C|A B, B| A\}$ p-entails $C \mid A$. By (5), as $\overline{A B} A B=\bar{A} A B C=\emptyset$, it holds that

$(C \mid A B) \wedge(B \mid A)=A B C+z \bar{A}$,

where $z=\mathbb{P}[(C \mid A B) \wedge(B \mid A)]$. Moreover, $B C \mid A=A B C+$ $x \bar{A}$, where $x=P(B C \mid A)$. As $(C \mid A B) \wedge(B \mid A)$ and $B C \mid A$ coincide conditionally on $A$ being true, by Theorem 3 , it follows that $(C \mid A B) \wedge(B \mid A)=B C \mid A$. Then, condition (ii) is satisfied, that is $(C \mid A B) \wedge(B \mid A) \wedge(C \mid A)=(B C \mid A) \wedge$ $(C \mid A)=B C \mid A=(C \mid A B) \wedge(B \mid A)$. Moreover, $C \mid A B \wedge$ $B|A=B C| A \leq C \mid A$, that is condition (iii) is satisfied too.

Remark 13 As shown in the analysis of Cut rule, it holds that $C|A B \wedge B| A=B C \mid A$. Then, the family $\{C|A B, B| A\}$ p-entails $B C \mid A$. This p-valid rule is called CCT (Conjunctive Cumulative Transitivity); see, e.g., [43].

CM rule The family $\{C|A, B| A\}$ p-entails $C \mid A B$. It holds that $(C \mid A) \wedge(B \mid A)=B C \mid A$. Moreover, $(C \mid A) \wedge(B \mid A) \wedge$ $(C \mid A B)=(B C \mid A) \wedge(C \mid A B)$. By (5), it holds that

$(B C \mid A) \wedge(C \mid A B)=A B C+z \bar{A}$,

where $z=\mathbb{P}[(B C \mid A) \wedge(C \mid A B)]$. Moreover, $B C \mid A=$ $A B C+x \bar{A}$, where $x=P(B C \mid A)$. As $(B C \mid A) \wedge(C \mid A B)$ and $B C \mid A$ coincide conditionally on $A$ being true, by Theorem 3 it follows that $(B C \mid A) \wedge(C \mid A B)=B C \mid A$; so that $(C \mid A) \wedge(B \mid A) \wedge(C \mid A B)=B C \mid A=(C \mid A) \wedge$ $(B \mid A)$, so that condition $(i i)$ is satisfied. Moreover, based on Definition 12, it holds that $(C \mid A) \wedge(B \mid A)=B C \mid A \subseteq$ 
$C \mid A B$, then $(C \mid A) \wedge(B \mid A) \leq C \mid A B$, so that condition (iii) is satisfied too.

Or rule The family $\{C|A, C| B\}$ p-entails $C \mid(A \vee B)$. We set $P(C \mid A)=x, P(C \mid B)=y$, and $\mathbb{P}((C \mid A) \wedge(C \mid B))=$ $z$; then, by observing that the family $\{A B C, \bar{A} B C, A \bar{B} C$, $(A \vee B) \bar{C}, \bar{A} \bar{B}\}$ is a partition of the sure event, we obtain

$(C \mid A) \wedge(C \mid B)=\left\{\begin{array}{l}1, \text { if } A B C \text { is true, } \\ 0, \text { if }(A \vee B) \bar{C} \text { is true, } \\ x, \text { if } \bar{A} B C \text { is true, } \\ y, \text { if } A \bar{B} C \text { is true, } \\ z, \text { if } \bar{A} \bar{B} \text { is true. }\end{array}\right.$

Moreover, by defining $\mathbb{P}[(C \mid A) \wedge(C \mid B) \wedge(C \mid(A \vee B))]=t$, we obtain

$(C \mid A) \wedge(C \mid B) \wedge(C \mid(A \vee B))= \begin{cases}1, & \text { if } A B C \text { is true, } \\ 0, & \text { if }(A \vee B) \bar{C} \text { is true, } \\ x, & \text { if } \bar{A} B C \text { is true, } \\ y, & \text { if } A \bar{B} C \text { is true, } \\ t, & \text { if } \bar{A} \bar{B} \text { is true. }\end{cases}$

As we can see, $(C \mid A) \wedge(C \mid B) \wedge(C \mid(A \vee B))$ and $(C \mid A) \wedge$ $(C \mid B)$ coincide when $A \vee B$ is true; then, by Theorem 3 it holds that $t=z$, so that

$(C \mid A) \wedge(C \mid B) \wedge(C \mid(A \vee B))=(C \mid A) \wedge(C \mid B)$,

that is condition (ii) is satisfied. Moreover, defining $P(C \mid(A \vee B))=w$, we have

$C \mid(A \vee B)= \begin{cases}1, & \text { if } A B C \text { is true, } \\ 0, & \text { if }(A \vee B) \bar{C} \text { is true, } \\ 1, & \text { if } \bar{A} B C \text { is true, } \\ 1, & \text { if } A \bar{B} C \text { is true, } \\ w, & \text { if } \bar{A} \bar{B} \text { is true. }\end{cases}$

Based on (23) and (24), it holds that $(C \mid A) \wedge(C \mid B) \leq$ $C \mid(A \vee B)$ conditionally on $A \vee B$ being true. Then, from Theorem 6 it holds that $\mathbb{P}((C \mid A) \wedge(C \mid B))=t \leq w=$ $P(C \mid(A \vee B))$; thus $(C \mid A) \wedge(C \mid B) \leq C \mid(A \vee B)$, that is condition $(i i i)$ is satisfied.

An inference rule related to Or rule $[1$, Rule 5, p. 189] In this inference rule the premise set is $\{C|(A \vee B), \bar{C}| A\}$ and the conclusion is $C \mid B$. We first observe that the premise set $\mathcal{F}=\{C|(A \vee B), \bar{C}| A\}$ is p-consistent because the assessment $P(C \mid(A \vee B))=P(\bar{C} \mid A)=1$ is coherent. Indeed, by applying Algorithm 1 to the pair $(\mathcal{F}, \mathcal{M})=$ $(\{C|(A \vee B), \bar{C}| A\},(1,1))$, it holds that the starting system $(\Sigma)$ is solvable, with $\mathcal{F}_{0}=\{\bar{C} \mid A\}$. Then, by repeating the steps of the algorithm, the assessment $P(\bar{C} \mid A)=1$ is coherent. Thus, the assessment $(1,1)$ on $\mathcal{F}$ is coherent and hence $\mathcal{F}$ is p-consistent. We also note that, defining $P(C \mid(A \vee B))=x, P(\bar{C} \mid A)=y$, and $\mathbb{P}((C \mid(A \vee$ $B) \wedge(\bar{C} \mid A))=z$, the coherence of $(x, y)=(1,1)$ from
(15) amounts to coherence of $z=1$, which by Theorem 17 is another characterization for the p-consistency of $\mathcal{F}$. Concerning p-entailment, we observe that

$$
\begin{aligned}
(C \mid A \vee B) \wedge(\bar{C} \mid A)=\left\{\begin{array}{l}
0, \text { if } A B C \text { is true, } \\
0, \text { if } A B \bar{C} \text { is true, } \\
0, \text { if } A \bar{B} C \text { is true, } \\
0, \text { if } A \bar{B} \bar{C} \text { is true, } \\
y, \text { if } \bar{A} B C \text { is true, } \\
0, \text { if } \bar{A} B \bar{C} \text { is true, } \\
z, \text { if } \bar{A} \bar{B} \text { is true, }
\end{array}\right. \\
=\left\{\begin{array}{l}
0, \text { if } A \vee \bar{A} B \bar{C} \text { is true, } \\
y, \text { if } \bar{A} B C \text { is true, } \\
z, \text { if } \bar{A} \bar{B} \text { is true. }
\end{array}\right.
\end{aligned}
$$

Moreover, by defining $\mathbb{P}[(C \mid(A \vee B)) \wedge(\bar{C} \mid A) \wedge(C \mid B)]=t$, we obtain

$(C \mid(A \vee B)) \wedge(\bar{C} \mid A) \wedge(C \mid B)= \begin{cases}0, & \text { if } A \vee \bar{A} B \bar{C} \text { is true, } \\ y, & \text { if } \bar{A} B C \text { is true, } \\ t, & \text { if } \bar{A} \bar{B} \text { is true. }\end{cases}$

As we can see from (25) and (26), the two quantities $(C \mid(A \vee B)) \wedge(\bar{C} \mid A) \wedge(C \mid B)$ and $(C \mid(A \vee B)) \wedge(\bar{C} \mid A)$ coincide when $A \vee B$ is true; then, by Theorem 3 it holds that $t=z$, so that

$(C \mid(A \vee B)) \wedge(\bar{C} \mid A) \wedge(C \mid B)=(C \mid(A \vee B)) \wedge(\bar{C} \mid A)$,

that is condition (ii) is satisfied. Moreover, defining $P(C \mid B)=w$, we have

$C \mid B=\left\{\begin{array}{ll}1, & \text { if } B C \text { is true, } \\ 0, & \text { if } B \bar{C} \text { is true, } \\ w, & \text { if } \bar{B} \text { is true. }\end{array}= \begin{cases}1, & \text { if } A B C \text { is true, } \\ 0, & \text { if } A B \bar{C} \text { is true, } \\ w, & \text { if } A \bar{B} C \text { is true, } \\ w, & \text { if } A \bar{B} \bar{C} \text { is true, } \\ 1, & \text { if } \bar{A} B C \text { is true, } \\ 0, & \text { if } \bar{A} B \bar{C} \text { is true, } \\ w, & \text { if } \bar{A} \bar{B} \text { is true, }\end{cases}\right.$

Based on (25) and (27), it holds that $(C \mid(A \vee B)) \wedge(\bar{C} \mid A) \leq$ $C \mid B$ conditionally on $A \vee B$ being true. Then, from Theorem 6 it holds that $\mathbb{P}((C \mid(A \vee B)) \wedge(\bar{C} \mid A))=t \leq w=P(C \mid B)$; thus $(C \mid(A \vee B)) \wedge(\bar{C} \mid A) \leq C \mid B$, that is condition (iii) is satisfied. Thus, this inference rule is p-valid. Notice that the p-validity of the rule could be also derived by using the lower and upper bounds given for Or rule in [18]. Indeed, using Or rule, when $P(C \mid A)=0$ and $P(C \mid B)=y$ it holds that $z=P(C \mid A \vee B) \in[0, y]$, so that $P(C \mid(A \vee B)) \leq$ $P(C \mid B)$. Then, $P(C \mid(A \vee B))=1$ and $P(\bar{C} \mid A)=1$ implies $P(C \mid B)=1$, that is $\{C|(A \vee B), \bar{C}| A\}$ p-entails $C \mid B$.

Generalized Or rule In this p-valid rule, studied in [19] (see also [25]), the p-consistent premise set is $\left\{C \mid A_{1}\right.$, $\left.C\left|A_{2}, \ldots, C\right| A_{n}\right\}$ and the conclusion is $C \mid\left(A_{1} \vee A_{2} \vee \cdots \vee\right.$ 
$\left.A_{n}\right)$. For each nonempty subset $S \subset\{1,2, \ldots, n\}$, we define $\mathbb{P}\left[\bigwedge_{i \in S}\left(C \mid A_{i}\right)\right]=x_{S} ;$ moreover, we set $\mathbb{P}\left[\bigwedge_{i=1}^{n}\left(C \mid A_{i}\right)\right]=$ $z$. Then,

$\left(C \mid A_{1}\right) \wedge \cdots \wedge\left(C \mid A_{n}\right)= \begin{cases}1, & \text { if } A_{1} A_{2} \cdots A_{n} C \text { is true, } \\ 0, & \text { if }\left(A_{1} \vee A_{2} \vee \cdots \vee A_{n}\right) \bar{C} \text { is true, } \\ x_{S}, & \text { if } \bigwedge_{i \in S} \bar{A}_{i} \bigwedge_{j \notin S} A_{j} C \text { is true, } \\ z, & \text { if } \bar{A}_{1} \bar{A}_{2} \cdots \bar{A}_{n} \text { is true. }\end{cases}$

Moreover, by defining $\mathbb{P}\left[\left(C \mid A_{1}\right) \wedge \cdots \wedge\left(C \mid A_{n}\right) \wedge\left(C \mid\left(A_{1} \vee\right.\right.\right.$ $\left.\left.\left.A_{2} \vee \cdots \vee A_{n}\right)\right)\right]=t$, we obtain

$$
\begin{aligned}
& \left(C \mid A_{1}\right) \wedge \cdots \wedge\left(C \mid A_{n}\right) \wedge\left(C \mid\left(A_{1} \vee A_{2} \vee \cdots \vee A_{n}\right)\right) \\
& = \begin{cases}1, & \text { if } A_{1} A_{2} \cdots A_{n} C \text { is true, } \\
0, & \text { if }\left(A_{1} \vee A_{2} \vee \cdots \vee A_{n}\right) \bar{C} \text { is true, } \\
x_{S}, & \text { if } \bigwedge_{i \in S} \bar{A}_{i} \bigwedge_{j \notin S} A_{j} C \text { is true, } \\
t, & \text { if } \bar{A}_{1} \bar{A}_{2} \cdots \bar{A}_{n} \text { is true. }\end{cases}
\end{aligned}
$$

As we can see from (28) and (29), $\left(C \mid A_{1}\right) \wedge \cdots \wedge\left(C \mid A_{n}\right) \wedge$ $\left(C \mid\left(A_{1} \vee A_{2} \vee \cdots \vee A_{n}\right)\right)$ and $\left(C \mid A_{1}\right) \wedge \cdots \wedge\left(C \mid A_{n}\right)$ coincide when $A_{1} \vee \cdots \vee A_{n}$ is true; then, by Theorem 3 it holds that $t=z$, so that

$$
\begin{aligned}
& \left(C \mid A_{1}\right) \wedge \cdots \wedge\left(C \mid A_{n}\right) \wedge\left(C \mid\left(A_{1} \vee A_{2} \vee \cdots \vee A_{n}\right)\right) \\
& \quad=\left(C \mid A_{1}\right) \wedge \cdots \wedge\left(C \mid A_{n}\right)
\end{aligned}
$$

that is condition $(i i)$ is satisfied. Moreover,

$C \mid\left(A_{1} \vee A_{2} \vee \cdots \vee A_{n}\right)= \begin{cases}1, & \text { if } A_{1} A_{2} \cdots A_{n} C \text { is true, } \\ 0, & \text { if }\left(A_{1} \vee A_{2} \vee \cdots \vee A_{n}\right) \bar{C} \text { is true, } \\ 1, & \text { if } \bigwedge_{i \in S} \bar{A}_{i} \bigwedge_{j \notin S} A_{j} C \text { is true, } \\ w, & \text { if } \bar{A}_{1} \bar{A}_{2} \cdots \bar{A}_{n} \text { is true, }\end{cases}$

where $w=P\left(C \mid\left(A_{1} \vee A_{2} \vee \cdots \vee A_{n}\right)\right)$. Based on (28) and (30), it holds that $\left(C \mid A_{1}\right) \wedge \cdots \wedge\left(C \mid A_{n}\right) \leq C \mid\left(A_{1} \vee A_{2} \vee \cdots \vee\right.$ $A_{n}$ ) conditionally on $A_{1} \vee \cdots \vee A_{n}$ being true. Then, from Theorem 6 it holds that $t \leq w$; thus $\left(C \mid A_{1}\right) \wedge \cdots \wedge\left(C \mid A_{n}\right) \leq$ $C \mid\left(A_{1} \vee A_{2} \vee \cdots \vee A_{n}\right)$, that is condition (iii) is satisfied.

\subsection{Iterated conditioning and p-entailment}

We now briefly describe a characterization of p-entailment of a conditional event $E_{3} \mid H_{3}$ from a p-consistent family $\left\{E_{1}\left|H_{1}, E_{2}\right| H_{2}\right\}$, which exploits a suitable notion of iterated conditioning.

Definition 14 Let be given $n+1$ conditional events $E_{1}\left|H_{1}, \ldots, E_{n+1}\right| H_{n+1}$, with $\left(E_{1} \mid H_{1}\right) \wedge \cdots \wedge\left(E_{n} \mid H_{n}\right) \neq 0$. We denote by $\left(E_{n+1} \mid H_{n+1}\right) \mid\left(\left(E_{1} \mid H_{1}\right) \wedge \cdots \wedge\left(E_{n} \mid H_{n}\right)\right)=$ $\left(E_{n+1} \mid H_{n+1}\right) \mid \mathcal{C}_{n}$ the random quantity

$\left(E_{1} \mid H_{1}\right) \wedge \cdots \wedge\left(E_{n+1} \mid H_{n+1}\right)+\mu\left(1-\left(E_{1} \mid H_{1}\right) \wedge \cdots \wedge\left(E_{n} \mid H_{n}\right)\right)$ $=\mathcal{C}_{n+1}+\mu\left(1-\mathcal{C}_{n}\right)$,

where $\mu=\mathbb{P}\left[\left(E_{n+1} \mid H_{n+1}\right) \mid \mathcal{C}_{n}\right]$.
We observe that, based on the betting metaphor, the quantity $\mu$ is the amount to be paid in order to receive the amount $\mathcal{C}_{n+1}+\mu\left(1-\mathcal{C}_{n}\right)$. Definition 14 generalizes the notion of iterated conditional $\left(E_{2} \mid H_{2}\right) \mid\left(E_{1} \mid H_{1}\right)$ given in previous papers (see, e.g., [22, 23, 26]). We also observe that, defining $\mathbb{P}\left(\mathcal{C}_{n}\right)=z_{n}$ and $\mathbb{P}\left(\mathcal{C}_{n+1}\right)=z_{n+1}$, by the linearity of prevision it holds that $\mu=z_{n+1}+\mu\left(1-z_{n}\right)$; then, $z_{n+1}=\mu z_{n}$, that is $\mathbb{P}\left(\mathcal{C}_{n+1}\right)=\mathbb{P}\left[\left(E_{n+1} \mid H_{n+1}\right) \mid \mathcal{C}_{n}\right] \mathbb{P}\left(\mathcal{C}_{n}\right)$, which is the compound prevision theorem.

By applying Definition 14 with $n=2$, given a p-consistent family $\left\{E_{1}\left|H_{1}, E_{2}\right| H_{2}\right\}$ and a further event $E_{3} \mid H_{3}$, it can be proved that [30]

$$
\begin{aligned}
\left\{E_{1}\left|H_{1}, E_{2}\right| H_{2}\right\} \text { p-entails } E_{3} \mid H_{3} & \Longleftrightarrow \\
\left(E_{3} \mid H_{3}\right) \mid\left(\left(E_{1} \mid H_{1}\right) \wedge\left(E_{2} \mid H_{2}\right)\right) & =1,
\end{aligned}
$$

that is: $\left\{E_{1}\left|H_{1}, E_{2}\right| H_{2}\right\}$ p-entails $E_{3} \mid H_{3}$ if and only if the iterated conditional $\left(E_{3} \mid H_{3}\right) \mid\left(\left(E_{1} \mid H_{1}\right) \wedge\left(E_{2} \mid H_{2}\right)\right)$ is constant and equal to 1 .

\section{From non $p$-valid to $p$-valid inference rules}

In this section we first examine some non p-valid inference rules, by showing that conditions (ii) and (iii) of Theorem 18 are not satisfied. Then, we illustrate by an example two different methods which allow to get $\mathrm{p}$-valid inference rules starting by non p-valid ones.

\subsection{Some non $p$-valid inference rules}

We start by showing that Transitivity is not $\mathrm{p}$-valid.

Transitivity In this rule the p-consistent premise set is $\{C|B, B| A\}$ and the conclusion is $C \mid A$. The rule is not p-valid [28], indeed we can show that

$(C \mid B) \wedge(B \mid A) \wedge(C \mid A) \neq(C \mid B) \wedge(B \mid A)$ and

$(C \mid B) \wedge(B \mid A) \not \leq C \mid A$.

Defining $P(B \mid A)=x, P(B C \mid A)=y, P(C \mid A)=t$, $\mathbb{P}((C \mid B) \wedge(B \mid A) \wedge(C \mid A))=\mu, \mathbb{P}((C \mid B) \wedge(B \mid A))=z$, we have

$(C \mid B) \wedge(B \mid A) \wedge(C \mid A)=(C \mid B) \wedge(B C \mid A)$

$$
= \begin{cases}1, & \text { if } A B C \text { is true, } \\ 0, & \text { if } A B \bar{C} \text { is true, } \\ 0, & \text { if } A \bar{B} C \text { is true, } \\ 0, & \text { if } A \bar{B} \bar{C} \text { is true, } \\ y, & \text { if } \bar{A} B C \text { is true, } \\ 0, & \text { if } \bar{A} B \bar{C} \text { is true, } \\ \mu, & \text { if } \bar{A} \bar{B} \text { is true, }\end{cases}
$$


and

$$
(C \mid B) \wedge(B \mid A)=\left\{\begin{array}{l}
1, \text { if } A B C \text { is true, } \\
0, \text { if } A B \bar{C} \text { is true, } \\
0, \text { if } A \bar{B} C \text { is true, } \\
0, \text { if } A \bar{B} \bar{C} \text { is true, } \\
x, \text { if } \bar{A} B C \text { is true, } \\
0, \text { if } \bar{A} B \bar{C} \text { is true, } \\
z, \text { if } \bar{A} \bar{B} \text { is true. }
\end{array}\right.
$$

Then, as (in general) $x \neq y$, it holds that $(C \mid B) \wedge(B \mid A) \wedge$ $(C \mid A) \neq(C \mid B) \wedge(B \mid A)$, so that condition (ii) is not satisfied. Moreover,

$$
C \mid A=\left\{\begin{array}{l}
1, \text { if } A B C \text { is true, } \\
0, \text { if } A B \bar{C} \text { is true, } \\
0, \text { if } A \bar{B} C \text { is true, } \\
0, \text { if } A \bar{B} \bar{C} \text { is true, } \\
t, \text { if } \bar{A} B C \text { is true, } \\
t, \text { if } \bar{A} B \bar{C} \text { is true, } \\
t, \text { if } \bar{A} \bar{B} \text { is true. }
\end{array}\right.
$$

Then, by observing that (in general) $x \nless t$ it follows that $(C \mid B) \wedge(B \mid A) \not \leq C \mid A$, so that condition (iii) is not satisfied. Therefore, Transitivity rule is not p-valid.

Denial of the antecedent We consider the rule where the premise set is $\{\bar{A}, C \mid A\}$ and the conclusion is $\bar{C}$. The premise set $\{\bar{A}, C \mid A\}$ is p-consistent because, by applying the Algorithm 1, the assessment $P(\bar{A})=P(C \mid A)=1$ is coherent. We verify that $\bar{A} \wedge(C \mid A) \wedge \bar{C} \neq \bar{A} \wedge(C \mid A)$ and that $\bar{A} \wedge(C \mid A) \not \leq \bar{C}$, that is the Denial of the antecedent is not p-valid. We set $P(C \mid A)=y$, then

$\bar{A} \wedge(C \mid A) \wedge \bar{C}=\left\{\begin{array}{l}0, \text { if } A \text { is true, } \\ 0, \text { if } \bar{A} C \text { is true, } \\ y, \text { if } \bar{A} \bar{C} \text { is true, }\end{array}\right.$

and

$\bar{A} \wedge(C \mid A)=\left\{\begin{array}{l}0, \text { if } A \text { is true, } \\ y, \text { if } \bar{A} C \text { is true, } \\ y, \text { if } \bar{A} \bar{C} \text { is true. }\end{array}\right.$

Assuming $y>0$, when $\bar{A} C$ is true it holds that

$\bar{A} \wedge(C \mid A) \wedge \bar{C}=0<y=\bar{A} \wedge(C \mid A), \quad \bar{A} \wedge(C \mid A)=y>0=\bar{C}$, thus: $\bar{A} \wedge(C \mid A) \wedge \bar{C} \neq \bar{A} \wedge(C \mid A)$ and $\bar{A} \wedge(C \mid A) \not \leq \bar{C}$, that is conditions (ii) and (iii) are not satisfied.

Affirmation of the consequent We consider the rule where the (p-consistent) premise set is $\{C, C \mid A\}$ and the conclusion is $A$. We verify that $C \wedge(C \mid A) \wedge A \neq C \wedge(C \mid A)$ and $C \wedge(C \mid A) \not \leq A$, that is the Affirmation of the consequent rule is not p-valid. We set $P(C \mid A)=y$, then

$C \wedge(C \mid A) \wedge A=A C=\left\{\begin{array}{l}1, \text { if } A C \text { is true, } \\ 0, \text { if } \overline{A C} \text { is true, }\end{array}\right.$ and

$C \wedge(C \mid A)=\left\{\begin{array}{l}1, \text { if } A C \text { is true, } \\ 0, \text { if } \bar{C} \text { is true, } \\ y, \text { if } \bar{A} C \text { is true. }\end{array}\right.$

Assuming $y>0$, when $\bar{A} C$ is true it holds that

$C \wedge(C \mid A) \wedge A=0<y=C \wedge(C \mid A)$,

$C \wedge(C \mid A)=y>0=A$,

thus: $C \wedge(C \mid A) \wedge A \neq C \wedge(C \mid A)$ and $C \wedge(C \mid A) \not \leq A$, that is conditions (ii) and (iii) are not satisfied.

Remark 14 We now will make a comparison between the two objects $C \wedge(C \mid A)$ and $C \mid(A \vee \bar{C})$, by showing they do not coincide. Defining $P(C \mid(A \vee \bar{C}))=t$, it holds that

$C \mid(A \vee \bar{C})=\left\{\begin{array}{l}1, \text { if } A C \text { is true, } \\ 0, \text { if } \bar{C} \text { is true, } \\ t, \text { if } \bar{A} C \text { is true. }\end{array}\right.$

It could seem, from (34) and (35), that $y$ and $t$ should be equal and then $C \wedge(C \mid A)$ and $C \mid(A \vee \bar{C})$ should coincide. However, in this case the conditioning event for $C \wedge(C \mid A)$ is $\Omega \vee A=\Omega$, so that the disjunction of the conditioning events is $\Omega \vee(A \vee \bar{C})=\Omega$; the two objects $C \wedge(C \mid A)$ and $C \mid(A \vee \bar{C})$ do not coincide conditionally on $\Omega$; then $C \wedge(C \mid A)$ and $C \mid(A \vee \bar{C})$ do not coincide (condition $(i)$ of Theorem 3 is not satisfied). We also observe that, defining $\mathbb{P}(C \wedge(C \mid A))=$ $\mu$, (in general) $\mu$ does not belong to the set $\{1,0, y\}$ of possible values of $C \wedge(C \mid A)$, because $\mu$ is a linear convex combination of the values $\{1,0, y\}$. As a further aspect, we verify below that $t \leq \mu \leq y$. The constituents generated by $\{A, C\}$ are: $A C, A \bar{C}, \bar{A} \bar{C}, \bar{A} \bar{C}$; then, the associated values for the random vector $(C|(A \vee \bar{C}), C \wedge(C \mid A), C| A)$ are

$$
(1,1,1), \quad(0,0,0), \quad(t, y, y), \quad(0,0, y) .
$$

Based on Theorem 6, we observe that:

- $C|(A \vee \bar{C}) \leq C| A$ conditionally on $A \vee \bar{C} \vee A=A \vee \bar{C}$, hence $P(C \mid(A \vee \bar{C}))=t \leq y=P(C \mid A)$;

- $C \wedge(C \mid A) \leq C \mid A$ conditionally on $\Omega \vee A=\Omega$, hence $\mathbb{P}(C \wedge(C \mid A))=\mu \leq y=P(C \mid A)$;

- $\quad C \mid(A \vee \bar{C}) \leq C \wedge(C \mid A)$ conditionally on $A \vee \bar{C} \vee \Omega=\Omega$, hence $P(C \mid(A \vee \bar{C}))=t \leq \mu=\mathbb{P}(C \wedge(C \mid A))$.

In other words: $t \leq \mu \leq y$. We observe that these inequalities also follow because coherence requires that the prevision point $(t, \mu, y)$ must be a linear convex combination of points in (36).

On combining evidence: an example from Boole We now examine an example studied in [8, p. 632] (see also [33, Theorem 5.45]), where p-entailment does not hold. Indeed, it can be proved that the extension $w=P(C \mid A B)$ of any (coherent) assessment $(x, y)$ on $\{C|A, C| B\}$ is 
coherent for every $w \in[0,1]$. Using conditions (ii) and (iii) of Theorem 18, we show that the p-consistent family $\{C|A, C| B\}$ does not p-entail $C \mid A B$. We set $P(C \mid A)=x$, $P(C \mid B)=y$, and $\mathbb{P}((C \mid A) \wedge(C \mid B))=z$; then,

$(C \mid A) \wedge(C \mid B)=\left\{\begin{array}{l}1, \text { if } A B C \text { is true, } \\ 0, \text { if } A B \bar{C} \text { is true, } \\ 0, \text { if } A \bar{B} \bar{C} \text { is true, } \\ 0, \text { if } \bar{A} B \bar{C} \text { is true, } \\ x, \text { if } \bar{A} B C \text { is true, } \\ y, \text { if } A \bar{B} C \text { is true, } \\ z, \text { if } \bar{A} \bar{B} \text { is true. }\end{array}=\left\{\begin{array}{l}1, \text { if } A B C \text { is true, } \\ 0, \text { if } A \vee B) \bar{C} \text { is true, } \\ x, \text { if } \bar{A} B \text { is true, } \\ y, \text { if } A \bar{B} C \text { is true, } \\ z, \text { if } \bar{A} \text { is true. }\end{array}\right.\right.$

Moreover, by defining $\mathbb{P}[(C \mid A) \wedge(C \mid A B)]=u, \mathbb{P}[(C \mid B) \wedge$ $(C \mid A B)]=v$ and $\mathbb{P}[(C \mid A) \wedge(C \mid B) \wedge(C \mid A B)]=t$, we obtain

$(C \mid A) \wedge(C \mid B) \wedge(C \mid A B)= \begin{cases}1, & \text { if } A B C \text { is true, } \\ 0, & \text { if }(A \vee B) \bar{C} \text { is true, } \\ u, & \text { if } \bar{A} B C \text { is true, } \\ v, & \text { if } A \bar{B} C \text { is true, } \\ t, & \text { if } \bar{A} \bar{B} \text { is true. }\end{cases}$

As in general $x \neq u$ and $y \neq v$, then $(C \mid A) \wedge(C \mid B) \wedge$ $(C \mid A B)$ and $(C \mid A) \wedge(C \mid B)$ do not coincide, so that condition $(i i)$ is not satisfied. Moreover,

$C \mid A B=\left\{\begin{array}{l}1, \text { if } A B C \text { is true, } \\ 0, \text { if } A B \bar{C} \text { is true, } \\ w, \text { if } A \bar{B} \bar{C} \text { is true, } \\ w, \text { if } \bar{A} B \bar{C} \text { is true, } \\ w, \text { if } \bar{A} B C \text { is true, } \\ w, \text { if } A \bar{B} C \text { is true, } \\ w, \text { if } \bar{A} \bar{B} \text { is true, }\end{array}=\left\{\begin{array}{l}1, \text { if } A B C \text { is true, } \\ 0, \text { if } A B \bar{C} \text { is true, } \\ w, \text { if } \overline{A B} \text { is true. }\end{array}\right.\right.$

Based on (37) and (38), we can see that $(C \mid A) \wedge(C \mid B) \not \leq$ $C \mid(A B)$, so that condition (iii) is not satisfied. Thus, the inference from $\{C|A, C| B\}$ to $C \mid A B$ is not p-valid.

\subsection{Two methods for constructing p-valid inference rules}

We now illustrate by an example two different methods by means of which, starting by a non p-valid inference rule, we get $\mathrm{p}$-valid inference rules: a) to add a suitable premise; b) to add a suitable logical constraint. The further premise, or logical constraint, (must preserve p-consistency and) is determined by analyzing the possible values of conjunctions.

Weak Transitivity In our example we start by the (non pvalid) Transitivity rule where the premise set is $\{C|B, B| A\}$ and the conclusion is $C \mid A$.
Method a). We add the premise $A \mid(A \vee B)$, so that the premise set is $\{C|B, B| A, A \mid(A \vee B)\}$, while the conclusion is still $C \mid A$. The premise set $\{C|B, B| A, A \mid(A \vee B)\}$ is pconsistent; indeed as $A B C \neq \emptyset$, by evaluating $P(A B C)=$ 1 we get $P(C \mid B)=P(B \mid A)=P(A \mid(A \vee B))=1$. We show that $(C \mid B) \wedge(B \mid A) \wedge(A \mid(A \vee B)) \wedge(C \mid A)=(C \mid B) \wedge$ $(B \mid A) \wedge(A \mid(A \vee B))$ and $(C \mid B) \wedge(B \mid A) \wedge(A \mid(A \vee B)) \leq$ $C \mid A$.

Defining $\mathbb{P}[(C \mid B) \wedge(B \mid A) \wedge(A \mid(A \vee B)) \wedge(C \mid A)]=\mu$, we have

$(C \mid B) \wedge(B \mid A) \wedge(A \mid(A \vee B)) \wedge(C \mid A)= \begin{cases}1, & \text { if } A B C \text { is true, } \\ 0, & \text { if } A B \bar{C} \text { is true, } \\ 0, & \text { if } A \bar{B} C \text { is true, } \\ 0, & \text { if } A \bar{B} \bar{C} \text { is true, } \\ 0, & \text { if } \bar{A} B C \text { is true, } \\ 0, & \text { if } \bar{A} B \bar{C} \text { is true, } \\ \mu, & \text { if } \bar{A} \bar{B} \text { is true. }\end{cases}$

Moreover, defining $\mathbb{P}[(C \mid B) \wedge(B \mid A) \wedge(A \mid(A \vee B))]=z$, we have

$(C \mid B) \wedge(B \mid A) \wedge(A \mid(A \vee B))= \begin{cases}1, & \text { if } A B C \text { is true, } \\ 0, \text { if } A B \bar{C} \text { is true, } \\ 0, \text { if } A \bar{B} C \text { is true, } \\ 0, \text { if } A \bar{B} \bar{C} \text { is true, } \\ 0, \text { if } \bar{A} B C \text { is true, } \\ 0, \text { if } \bar{A} B \bar{C} \text { is true, } \\ z, \text { if } \bar{A} \bar{B} \text { is true. }\end{cases}$

Conditionally on $A \vee B$ being true it holds that $(C \mid B) \wedge$ $(B \mid A) \wedge(A \mid(A \vee B)) \wedge(C \mid A)=(C \mid B) \wedge(B \mid A) \wedge(A \mid(A \vee$ $B))=A B C \mid(A \vee B)$. Then, by Theorem 3 we have $(C \mid B) \wedge(B \mid A) \wedge(A \mid(A \vee B)) \wedge(C \mid A)=(C \mid B) \wedge(B \mid A) \wedge$ $(A \mid(A \vee B))=A B C \mid(A \vee B)$, so that condition (ii) is satisfied. Finally, as $A B C|(A \vee B) \subseteq C| A$, it holds that $(C \mid B) \wedge(B \mid A) \wedge(A \mid(A \vee B))=A B C|(A \vee B) \leq C| A$, so that condition (iii) is satisfied. Therefore this Weak Transitivity rule is $p$-valid. We observe that another p-valid version of Weak Transitivity would be obtained by adding the premise $A \mid B$ instead of $A \mid(A \vee B)$.

Method b). We add the logical constraint $\bar{A} B C=\emptyset$, that is $B C \subseteq A$. The p-consistency of the premise set $\{C|B, B| A\}$ is preserved because, as before $A B C \neq \emptyset$ and by evaluating $P(A B C)=1$ we get $P(C \mid B)=P(B \mid A)=1$. Based on (31), (32), (33) it holds that

$$
\begin{aligned}
(C \mid B) \wedge(B \mid A) \wedge(C \mid A)= & (C \mid B) \wedge(B C \mid A) \\
= & \begin{cases}1, & \text { if } A B C \text { is true }, \\
0, & \text { if } A B \bar{C} \text { is true, } \\
0, & \text { if } A \bar{B} C \text { is true, } \\
0, & \text { if } A \bar{B} \bar{C} \text { is true }, \\
0, & \text { if } \bar{A} B \bar{C} \text { is true } \\
\mu, & \text { if } \bar{A} \bar{B} \text { is true },\end{cases}
\end{aligned}
$$


and

$$
(C \mid B) \wedge(B \mid A)=\left\{\begin{array}{l}
1, \text { if } A B C \text { is true, } \\
0, \text { if } A B \bar{C} \text { is true, } \\
0, \text { if } A \bar{B} C \text { is true, } \\
0, \text { if } A \bar{B} \bar{C} \text { is true, } \\
0, \text { if } \bar{A} B \bar{C} \text { is true, } \\
z, \text { if } \bar{A} \bar{B} \text { is true. }
\end{array}\right.
$$

As we can see $(C \mid B) \wedge(B \mid A) \wedge(C \mid A)=(C \mid B) \wedge(B \mid A)$ conditionally on $A \vee B$ being true. Then, by Theorem 3 condition (ii) is satisfied. Moreover,

$C \mid A=\left\{\begin{array}{l}1, \text { if } A B C \text { is true, } \\ 0, \text { if } A B \bar{C} \text { is true, } \\ 0, \text { if } A \bar{B} C \text { is true, } \\ 0, \text { if } A \bar{B} \bar{C} \text { is true, } \\ t, \text { if } \bar{A} B C \text { is true, } \\ t, \text { if } \bar{A} \bar{B} \text { is true. }\end{array}\right.$

Then, $(C \mid B) \wedge(B \mid A) \leq C \mid A$ conditionally on $A \vee B$ being true. Thus, by Theorem 6 condition (iii) is satisfied too. Therefore, under the logical constraint $\bar{A} B C=\emptyset$, the family $\{C|B, B| A\}$ p-entails $C \mid A$, which is another p-valid version of Weak Transitivity.

We observe that in [28, Theorem 5] it has been shown that another p-valid version of Weak Transitivity is obtained by adding the probabilistic constraint $P(A \mid(A \vee B))>0$, that is

$$
\begin{aligned}
& P(C \mid B)=1, P(B \mid A)=1, P(A \mid(A \vee B))>0 \\
& \Longrightarrow P(C \mid A)=1 .
\end{aligned}
$$

\section{Conclusions}

We generalized the notions of conjunction and disjunction of two conditional events to the case of $n$ conditional events. We introduced the notion of negation and we showed that De Morgan's Laws still hold. We also verified that the associative and commutative properties are satisfied. We studied the monotonicity property, by proving that $\mathcal{C}_{n+1} \leq$ $\mathcal{C}_{n}$ and $\mathcal{D}_{n+1} \geq \mathcal{D}_{n}$ for every $n$. We computed the set of all coherent assessments on the family $\left\{\mathcal{C}_{n}, E_{n+1} \mid H_{n+1}, \mathcal{C}_{n+1}\right\}$, by showing that Fréchet-Hoeffding bounds still hold in this case; then, we examined the (reverse) probabilistic inference from $\mathcal{C}_{n+1}$ to the family $\left\{\mathcal{C}_{n}, E_{n+1} \mid H_{n+1}\right\}$. Moreover, given a family $\mathcal{F}=\left\{E_{1}\left|H_{1}, E_{2}\right| H_{2}, E_{3} \mid H_{3}\right\}$ of three conditional events, with $E_{1}, E_{2}, E_{3}, H_{1}, H_{2}, H_{3}$ logically independent, we determined the set $\Pi$ of all coherent prevision assessments for the set of conjunctions $\left\{\mathcal{C}_{S}: \emptyset \neq\right.$ $S \subseteq\{1,2,3\}\}$. In particular, we verified that the set $\Pi$ is the same in the case where $H_{1}=H_{2}=H_{3}$ and we also considered the relation between conjunction and quasiconjunction. By using conjunction we also characterized p-consistency and p-entailment; then, we examined several examples of p-valid inference rules. We briefly described a characterization of p-entailment, in the case of two premises, by using a suitable notion of iterated conditioning. Then, after examining some non p-valid inference rules, we illustrated by an example two methods for constructing p-valid inference rules. In particular, we applied these methods to Transitivity by obtaining $\mathrm{p}$-valid versions of the rule (Weak Transitivity). Future work could concern the extension of the results of this paper to more complex cases, with possible applications to the psychology of cognitive reasoning under uncertainty. This work should lead, for instance, to further developments of the results given in [41, 42].

Acknowledgements We thank the anonymous referees for their useful criticisms and suggestions.

\section{Appendix A}

Proof of Theorem 5 We observe that (ii) follows by $(i)$, by replacing $\mathcal{F}$ by $\overline{\mathcal{F}}$; indeed, by $(i)$ it holds that $\overline{\mathcal{D}(\overline{\mathcal{F}})}=$ $\mathcal{C}(\overline{\overline{\mathcal{F}}})=\mathcal{C}(\mathcal{F})$. Then, it is enough to proof the assertion $(i)$. We will prove the assertion by induction.

Step 1: $n=1, \mathcal{F}=\left\{E_{1} \mid H_{1}\right\}$.

We have $\overline{\mathcal{D}(\mathcal{F})}=\overline{E_{1} \mid H_{1}}=1-E_{1} \mid H_{1}=$ $\bar{E}_{1} \mid H_{1}=\mathcal{C}(\overline{\mathcal{F}})$.

Thus the assertion holds when $n=1$.

Step 2: $\quad n=2, \mathcal{F}=\left\{E_{1}\left|H_{1}, E_{2}\right| H_{2}\right\}$.

We set

$$
\begin{aligned}
& P\left(E_{1} \mid H_{1}\right)=x, P\left(E_{2} \mid H_{2}\right)=y, \mathbb{P}\left[\left(E_{1} \mid H_{1}\right)\right. \\
& \left.\vee\left(E_{2} \mid H_{2}\right)\right]=w, \mathbb{P}\left[\left(\bar{E}_{1} \mid H_{1}\right) \wedge\left(\bar{E}_{2} \mid H_{2}\right)\right]=t .
\end{aligned}
$$

We observe that the family $\left\{E_{1} H_{1} \vee\right.$ $\left.E_{2} H_{2}, \bar{E}_{1} H \bar{E}_{2} H_{2}, \bar{H}_{1} \bar{E}_{2} H_{2}, \bar{E}_{1} H_{1} \bar{H}_{2}, \bar{H}_{1} \bar{H}_{2}\right\}$ is a partition of the sure event $\Omega$. Moreover, by Definitions 3 and 4 we have

$$
\begin{aligned}
\overline{\mathcal{D}(\mathcal{F})}= & 1-\left(E_{1} \mid H_{1}\right) \vee\left(E_{2} \mid H_{2}\right) \\
= & \begin{cases}0, & \text { if } E_{1} H_{1} \vee E_{2} H_{2} \text { is true, } \\
1, & \text { if } \bar{E}_{1} H_{1} \bar{E}_{2} H_{2} \text { is true, } \\
1-x, & \text { if } \bar{H}_{1} \bar{E}_{2} H_{2} \text { is true, } \\
1-y, & \text { if } \bar{E}_{1} H_{1} \bar{H}_{2} \text { is true, } \\
1-w, & \text { if } \bar{H}_{1} \bar{H}_{2} \text { is true. }\end{cases}
\end{aligned}
$$

and

$$
\begin{aligned}
\mathcal{C}(\overline{\mathcal{F}})= & \left(\bar{E}_{1} \mid H_{1}\right) \wedge\left(\bar{E}_{2} \mid H_{2}\right) \\
= & \begin{cases}0, & \text { if } E_{1} H_{1} \vee E_{2} H_{2} \text { is true, } \\
1, & \text { if } \bar{E}_{1} H \bar{E}_{2} H_{2} \text { is true, } \\
1-x, & \text { if } \bar{H}_{1} \bar{E}_{2} H_{2} \text { is true, } \\
1-y, & \text { if } \bar{E}_{1} H_{1} \bar{H}_{2} \text { is true, } \\
t, & \text { if } \bar{H}_{1} \bar{H}_{2} \text { is true. }\end{cases}
\end{aligned}
$$


We observe that $\overline{\mathcal{D}(\mathcal{F})}$ and $\mathcal{C}(\overline{\mathcal{F}})$ coincide when $H_{1} \vee H_{2}$ is true. Thus, by Theorem $3, \mathbb{P}(\overline{\mathcal{D}(\mathcal{F})})=$ $\mathbb{P}(\mathcal{C}(\overline{\mathcal{F}}))$ and hence $1-w=t$. Therefore $\overline{\mathcal{D}(\mathcal{F})}$ still coincides with $\mathcal{C}(\overline{\mathcal{F}})$ when $H_{1} \vee H_{2}$ is false, so that $\overline{\mathcal{D}(\mathcal{F})}=\mathcal{C}(\overline{\mathcal{F}})$.

Step 3: $\mathcal{F}=\left\{E_{1}\left|H_{1}, E_{2}\right| H_{2}, \ldots, E_{n} \mid H_{n}\right\}$.

(Inductive Hypothesis) Let us assume that for any (strict) subset $S \subset\{1, \ldots, n\}$, by defining $\mathcal{F}_{S}=\left\{E_{i} \mid H_{i}, i \in S\right\}$, it holds that $\overline{\mathcal{D}\left(\mathcal{F}_{S}\right)}=$ $\mathcal{C}\left(\overline{\mathcal{F}_{S}}\right)$. Now we will prove that $\overline{\mathcal{D}\left(\mathcal{F}_{S}\right)}=\mathcal{C}\left(\overline{\mathcal{F}_{S}}\right)$ when $S=\{1, \ldots, n\}$, in which case $\mathcal{F}_{S}=\mathcal{F}$. By Definition 7 we have

$$
\begin{aligned}
\overline{\mathcal{D}(\mathcal{F})} & =\sum_{h=0}^{m} w_{h} C_{h}, \text { where } \\
w_{h} & = \begin{cases}0, & \text { if } S_{h}^{\prime} \neq \emptyset, \\
1, & \text { if } S_{h}^{\prime \prime}=\{1,2, \ldots, n\}, \\
1-y_{S_{h}^{\prime \prime \prime}}, & \text { if } S_{h}^{\prime}=\emptyset \text { and } S_{h}^{\prime \prime \prime} \neq \emptyset .\end{cases}
\end{aligned}
$$

We continue to use the subsets $S_{h}^{\prime}, S_{h}^{\prime \prime}, S_{h}^{\prime \prime \prime}$ as defined in formula (9) also with the family $\overline{\mathcal{F}}$; moreover we set $t_{S}=\mathbb{P}\left[\bigwedge_{i \in S}\left(\bar{E}_{i} \mid H_{i}\right)\right]=$ $\mathbb{P}\left[\mathcal{C}\left(\overline{\mathcal{F}}_{S}\right)\right]$. Based on Definition 5, we have

$$
\begin{aligned}
\mathcal{C}(\overline{\mathcal{F}}) & =\sum_{h=0}^{m} z_{h} C_{h}, \text { where } \\
z_{h} & = \begin{cases}0, & \text { if } S_{h}^{\prime} \neq \emptyset, \\
1, & \text { if } S_{h}^{\prime \prime}=\{1,2, \ldots, n\}, \\
t_{S_{h}^{\prime \prime \prime}}, & \text { if } S_{h}^{\prime}=\emptyset \text { and } S_{h}^{\prime \prime \prime} \neq \emptyset .\end{cases}
\end{aligned}
$$

Then, $\overline{\mathcal{D}(\mathcal{F})}-\mathcal{C}(\overline{\mathcal{F}})=\sum_{h=0}^{m}\left(w_{h}-z_{h}\right) C_{h}$, where

$$
\begin{aligned}
& w_{h}-z_{h} \\
& \quad= \begin{cases}0, & \text { if } S_{h}^{\prime} \neq \emptyset, \\
0, & \text { if } S_{h}^{\prime \prime}=\{1,2, \ldots, n\}, \\
1-y_{S_{h}^{\prime \prime \prime}}-t_{S_{h}^{\prime \prime \prime}}, & \text { if } S_{h}^{\prime}=\emptyset \text { and } S_{h}^{\prime \prime \prime} \neq \emptyset .\end{cases}
\end{aligned}
$$

By the inductive hypothesis, it holds that $1-$ $y_{S_{h}^{\prime \prime \prime}}=\mathbb{P}\left[\overline{\mathcal{D}\left(\mathcal{F}_{S_{h}^{\prime \prime \prime}}\right)}\right]=\mathbb{P}\left[\mathcal{C}\left(\overline{\mathcal{F}}_{S_{h}^{\prime \prime \prime}}\right)\right]=t_{S_{h}^{\prime \prime \prime}}$ for $h=1, \ldots, m$, because $S_{h}^{\prime \prime \prime} \subset\{1,2, \ldots, n\}$. Then, $\overline{\mathcal{D}(\mathcal{F})}-\mathcal{C}(\overline{\mathcal{F}})=\sum_{h=0}^{m}\left(w_{h}-z_{h}\right) C_{h}$, where

$w_{h}-z_{h}= \begin{cases}0, & h=1, \ldots, m, \\ 1-y_{S_{0}^{\prime \prime \prime}}-t_{S_{0}^{\prime \prime \prime}}, & h=0 .\end{cases}$

By recalling that $S_{0}^{\prime \prime \prime}=\{1,2, \ldots, n\}, \overline{\mathcal{D}(\mathcal{F})}$ and $\mathcal{C}(\overline{\mathcal{F}})$ coincide when $H_{1} \vee H_{2} \vee \cdots \vee H_{n}$ is true. Thus, by Theorem 3, $\mathbb{P}[\overline{\mathcal{D}(\mathcal{F})}]=\mathbb{P}[\mathcal{C}(\overline{\mathcal{F}}))]$, that is $1-y_{S_{0}^{\prime \prime \prime}}=t_{S_{0}^{\prime \prime \prime}}$. Therefore $\overline{\mathcal{D}(\mathcal{F})}$ still coincides with $\mathcal{C}(\overline{\mathcal{F}})$ when $H_{1} \vee H_{2} \vee \cdots \vee H_{n}$ is false, so that $\overline{\mathcal{D}(\mathcal{F})}=\mathcal{C}(\overline{\mathcal{F}})$.
Proof of Theorem 6 (i) Assume that, for every $(\mu, v) \in$ $\Pi$, the values of $X \mid H$ and $Y \mid K$ associated with the constituent $C_{h}$ are such that $X|H \leq Y| K$, for each $C_{h}$ contained in $H \vee K$; then for each given coherent assessment $(\mu, v)$, by choosing $s_{1}=1, s_{2}=-1$ in the random gain, we have

$$
\begin{aligned}
G & =H(X-\mu)-K(Y-v)=(X \mid H-\mu)-(Y \mid K-v) \\
& =(X|H-Y| K)+(v-\mu) .
\end{aligned}
$$

Then, by the hypothesis, $\mathcal{G}_{H \vee K} \leq(\nu-\mu)$ and by coherence $0=\mathbb{P}\left(\mathcal{G}_{H \vee K}\right) \leq v-\mu$. Then $\mu \leq v$, $\forall(\mu, v) \in \Pi$.

(ii) By hypothesis, it holds that $\left(X H+\mu H^{c}\right)(H \vee K) \leq$ $\left(Y K+v K^{c}\right)(H \vee K)$; moreover, from condition (i), $\mu \leq v$ for every $(\mu, v) \in \Pi$; then

$$
\begin{aligned}
X \mid H= & X H+\mu \bar{H}=(X H+\mu \bar{H})(H \vee K) \\
& +(X H+\mu \bar{H}) \bar{H} \bar{K}=(X H+\mu \bar{H})(H \vee K) \\
& +\mu \bar{H} \bar{K} \leqslant(Y K+v \bar{K})(H \vee K)+v \overline{H K} \\
& =(Y K+v \bar{K})(H \vee K)+(Y K+v \bar{K}) \overline{H K} \\
& =Y K+v \bar{K}=Y \mid K .
\end{aligned}
$$

Vice versa, $X|H \leq Y| K$ trivially implies $X|H \leq Y| K$ when $H \vee K$ is true.

Proof of Theorem 7 We distinguish three cases: $(a)$ the value of $\mathcal{C}_{n}$ is 0 , with some $E_{i} \mid H_{i}$ false, $i \leq n ;(b)$ the value of $\mathcal{C}_{n}$ is 1 , with $E_{i} \mid H_{i}$ true, $i=1, \ldots, n$; $(c)$ the value of $\mathcal{C}_{n}$ is $\mathbb{P}\left[\bigwedge_{i \in S}\left(E_{i} \mid H_{i}\right)\right]=\mathbb{P}\left(\mathcal{C}_{S}\right)=x_{S}$, for some subset $S \subseteq\{1,2, \ldots, n\}$.

Case (a). It holds that $\mathcal{C}_{n+1}=0=\mathcal{C}_{n}$.

Case $(b)$. The value of $\mathcal{C}_{n+1}$ is 1 , or 0 , or $x_{n+1}$, according to whether $E_{n+1} \mid H_{n+1}$ is true, or false, or void; thus $\mathcal{C}_{n+1} \leq \mathcal{C}_{n}$.

Case (c). We distinguish three cases: (i) $E_{n+1} \mid H_{n+1}$ is true; (ii) $E_{n+1} \mid H_{n+1}$ is false; (iii) $E_{n+1} \mid H_{n+1}$ is void. In the case $(i)$ the value of $\mathcal{C}_{n+1}$ is $x_{S}$, thus $\mathcal{C}_{n+1}=\mathcal{C}_{n}$. In the case $(i i)$ the value of $\mathcal{C}_{n+1}$ is 0 , thus $\mathcal{C}_{n+1} \leq \mathcal{C}_{n}$. In the case (iii) the value of $\mathcal{C}_{n+1}$ is $x_{S \cup\{n+1\}}=$ $\mathbb{P}\left[\bigwedge_{i \in S \cup\{n+1\}}\left(E_{i} \mid H_{i}\right)\right]$; then, in order to prove that $\mathcal{C}_{n+1} \leq \mathcal{C}_{n}$, we need to prove that $x_{S \cup\{n+1\}} \leq x_{S}$. We proceed by induction on the cardinality of $S$, denoted by $s$. Let be $s=1$, with $\mathcal{C}_{S}=E_{i} \mid H_{i}$, for some $i \in\{1, \ldots, n\}$. We note that $x_{S}=\mathbb{P}\left(E_{i} \mid H_{i}\right)=x_{i}, x_{S \cup\{n+1\}}=$ $\mathbb{P}\left(\left(E_{i} \mid H_{i}\right) \wedge\left(E_{n+1} \mid H_{n+1}\right)\right)=x_{\{i, n+1\}}$ and by Theorem 4 it holds that $x_{S \cup\{n+1\}}=x_{\{i, n+1\}} \leq$ $x_{i}=x_{S}$. Now, let be $s \geq 2$ and $x_{S \cup\{n+1\}} \leq x_{S}$ for every $s<n$, so that, based on Definition 5 , $\mathcal{C}_{n+1} \leq \mathcal{C}_{n}$ when $S$ is a strict subset of $\{1,2, \ldots, n\}$. If $S=\{1,2, \ldots, n\}$, as $E_{i} \mid H_{i}$ is 
void for all $i=1, \ldots, n+1$, it holds that $\mathcal{C}_{n}=$ $\mathbb{P}\left(\mathcal{C}_{n}\right)=x_{\{1, \ldots, n\}}$ and $\mathcal{C}_{n+1}=x_{\{1, \ldots, n+1\}}=$ $\mathbb{P}\left(\mathcal{C}_{n+1}\right)$ and, in order to prove that $\mathcal{C}_{n+1} \leq \mathcal{C}_{n}$, it remains to prove that $\mathbb{P}\left(\mathcal{C}_{n+1}\right) \leq \mathbb{P}\left(\mathcal{C}_{n}\right)$. By applying Theorem 6, with $X \mid H=\mathcal{C}_{n+1}=$ $Z_{n+1} \mid\left(H_{1} \vee \cdots \vee H_{n+1}\right)$ and $Y \mid K=\mathcal{C}_{n}=$ $Z_{n} \mid\left(H_{1} \vee \cdots \vee H_{n}\right)$, as $\mathcal{C}_{n+1} \leq \mathcal{C}_{n}$ when $H_{1} \vee$ $\cdots \vee H_{n+1}$ is true (i.e., $\left.s<n\right)$, it follows that $\mathbb{P}\left(\mathcal{C}_{n+1}\right) \leq \mathbb{P}\left(\mathcal{C}_{n}\right)$; therefore $\mathcal{C}_{n+1} \leq \mathcal{C}_{n}$.

\section{Proof of Theorem 9.}

Case $(i)$. We proceed by induction. The property is satisfied for $n=1$; indeed, if $\mathcal{C}_{1}=E_{1} \mid H_{1} \in\left\{1,0, x_{1}\right\}$, where $x_{1}=\mathbb{P}\left(E_{1} \mid H_{1}\right) \in[0,1]$, then $\mathcal{C}_{1} \in[0,1]$. Let us assume that the property holds for $k<n$, that is $\mathcal{C}_{k} \in[0,1]$, for every $k<n$. Based on Definition 5 we distinguish three cases: $(a)$ the value of $\mathcal{C}_{n}$ is $0 ;(b)$ the value of $\mathcal{C}_{n}$ is $1 ;(c)$ the value of $\mathcal{C}_{n}$ is $\mathbb{P}\left[\bigwedge_{i \in S}\left(E_{i} \mid H_{i}\right)\right]=x_{S}$, for some subset $S \subseteq\{1,2, \ldots, n\}$. In the cases $(a)$ and $(b), \mathcal{C}_{n} \in[0,1]$. In the case $(c)$, if $S=\left\{i_{1}, \ldots, i_{k}\right\} \subset\{1,2, \ldots, n\}$, then $\mathcal{C}_{n} \in[0,1]$, because $x_{S}=\mathbb{P}\left(\bigwedge_{j=1}^{k}\left(E_{i_{j}} \mid H_{i_{j}}\right)\right)$ is a possible value of $\mathcal{C}_{k}=\bigwedge_{j=1}^{k}\left(E_{i_{j}} \mid H_{i_{j}}\right)$, with $k<n$. Finally, if $S=$ $\{1,2, \ldots, n\}$ (that is the conditioning events $H_{1}, \ldots, H_{n}$ are all false), then $\mathcal{C}_{n}=\mathbb{P}\left(\mathcal{C}_{n}\right)$ and $\mathbb{P}\left(\mathcal{C}_{n}\right) \in[0,1]$ because the values of $\mathcal{C}_{n}$ restricted to $H_{1} \vee \cdots \vee H_{n}$ all belong to [0, 1]. Therefore $\mathcal{C}_{n} \in[0,1]$. By a similar reasoning, based on Definition 7 we can prove that $\mathcal{D}_{n} \in[0,1]$.

Proof of Theorem 10 Let $C_{0}, \ldots, C_{m}$, with $m=3^{n}-1$ be the constituents associated with $\mathcal{F}_{n+1}=\left\{E_{1} \mid H_{1}, \ldots\right.$, $\left.E_{n+1} \mid H_{n+1}\right\}$, where $C_{0}=\bar{H}_{1} \cdots \bar{H}_{n+1}$. With each $C_{h}, h=1, \ldots, m$, we associate the point $Q_{h}=$ $\left(q_{h 1}, q_{h 2}, q_{h 3}\right)$, which represents the value of the random vector $\left(\mathcal{C}_{n}, E_{n+1} \mid H_{n+1}, \mathcal{C}_{n+1}\right)$ when $C_{h}$ is true, where $q_{h 1}$ is the value of $\mathcal{C}_{n}, q_{h 2}$ is the value of $E_{n+1} \mid H_{n+1}$, and $q_{h 3}$ is the value of $\mathcal{C}_{n+1}$. With $C_{0}$ it is associated the point $Q_{0}=\left(\mu_{n}, x_{n+1}, \mu_{n+1}\right)=\mathcal{M}$. We observe that the set of points $\left\{Q_{h}, h=1, \ldots, m\right\}$ contains in particular the points

$Q_{1}=(1,1,1), Q_{2}=(1,0,0), Q_{3}=(0,1,0), Q_{4}=(0,0,0)$,

which are respectively associated with the following constituents or logical disjunction of constituents

$E_{1} H_{1} \cdots E_{n} H_{n} E_{n+1} H_{n+1}, E_{1} H_{1} \cdots E_{n} H_{n} \bar{E}_{n+1} H_{n+1}$,

$\left(\bar{E}_{1} H_{1} \vee \cdots \vee \bar{E}_{n} H_{n}\right) \wedge E_{n+1} H_{n+1}$,

$\left(\bar{E}_{1} H_{1} \vee \cdots \vee \bar{E}_{n} H_{n}\right) \wedge \bar{E}_{n+1} H_{n+1}$.

Based on Remark 7, we need to prove that the set of coherent assessments $\Pi$ on $\left\{\mathcal{C}_{n}, E_{n+1} \mid H_{n+1}, \mathcal{C}_{n+1}\right\}$ coincides with the convex hull $\mathcal{I}$ of $Q_{1}, Q_{2}, Q_{3}, Q_{4}$. We recall that coherence of $\left(\mu_{n}, x_{n+1}, \mu_{n+1}\right)$ implies coherence of all the sub-assessments on the associated subfamilies of $\left\{\mathcal{C}_{n}, E_{n+1} \mid H_{n+1}, \mathcal{C}_{n+1}\right\}$. The coherence of the single assessments $\mu_{n}$ on $\mathcal{C}_{n}$, or $x_{n+1}$ on $E_{n+1} \mid H_{n+1}$, or $\mu_{n+1}$ on $\mathcal{C}_{n+1}$, simply amounts to conditions

$\mu_{n} \in[0,1], x_{n+1} \in[0,1], \mu_{n+1} \in[0,1]$,

respectively. Then, by the hypothesis of logical independence, the sub-assessment $\left(\mu_{n}, x_{n+1}\right)$ is coherent, for every $\left(\mu_{n}, x_{n+1}\right) \in[0,1]^{2}$. By Remark 6, the coherence of the sub-assessments $\left(\mu_{n}, \mu_{n+1}\right)$ and $\left(x_{n+1}, \mu_{n+1}\right)$ amounts to the conditions $0 \leq \mu_{n+1} \leq \mu_{n} \leq 1$ and $0 \leq \mu_{n+1} \leq$ $x_{n+1} \leq 1$. Finally, assuming that the above conditions are satisfied, to prove coherence of $\left(\mu_{n}, x_{n+1}, \mu_{n+1}\right)$, by Theorem 1 , it is enough to show that the point $\left(\mu_{n}, x_{n+1}, \mu_{n+1}\right)$ belongs to the convex hull of the points $Q_{1}, \ldots, Q_{m}$. Moreover, in order $\mathcal{M}$ belongs to the convex hull of $Q_{1}, \ldots, Q_{m}$ the following system $(\Sigma)$ must solvable

$\mathcal{M}=\sum_{h=1}^{m} \lambda_{h} Q_{h}, \quad \sum_{h=1}^{m} \lambda_{h}=1, \quad \lambda_{h} \geq 0, \forall h$.

We show that the convex hull of the points $Q_{1}, \ldots, Q_{m}$ coincides with the convex hull $\mathcal{I}$ of the points $Q_{1}, Q_{2}, Q_{3}, Q_{4}$, described in Remark 7, because all the other points $Q_{5}, \ldots, Q_{m}$, are linear convex combinations of $Q_{1}, Q_{2}, Q_{3}, Q_{4}$, that is $Q_{h} \in \mathcal{I}$ for each $h=5, \ldots, m$.

We examine the following different cases which depend on the logical value of $\left.E_{n+1} \mid H_{n+1}: a\right) E_{n+1} \mid H_{n+1}$ is true; b) $E_{n+1} \mid H_{n+1}$ is false; c) $E_{n+1} \mid H_{n+1}$ is void.

a) In this case

$$
\begin{aligned}
Q_{h} & =\left(q_{h 1}, 1, q_{h 1}\right)=q_{h 1}(1,1,1)+\left(1-q_{h 1}\right)(0,1,0) \\
& =q_{h 1} Q_{1}+\left(1-q_{h 1}\right) Q_{3} .
\end{aligned}
$$

b) In this case

$$
\begin{aligned}
Q_{h} & =\left(q_{h 1}, 0,0\right)=q_{h 1}(1,0,0)+\left(1-q_{h 1}\right)(0,0,0) \\
& =q_{h 1} Q_{2}+\left(1-q_{h 1}\right) Q_{4} .
\end{aligned}
$$

c) In this case $Q_{h}=\left(q_{h 1}, x_{n+1}, q_{h 3}\right)$ and we distinguish the following subcases: $(i) \bigwedge_{i=1}^{n} E_{i} H_{i}$ true, so that $Q_{h}=\left(1, x_{n+1}, x_{n+1}\right)$; (ii) $\bigvee_{i=1}^{n} \bar{E}_{i} H_{i}$ true, so that $Q_{h}=\left(0, x_{n+1}, 0\right)$; (iii) $E_{i} \mid H_{i}$ void, for every $i \in$ $S$ and $E_{i} \mid H_{i}$ true for every $i \in\{1,2, \ldots, n\} \backslash S$, for some $\emptyset \neq S \subset\{1,2, \ldots, n\}$, so that $Q_{h}=$ $\left(x_{S}, x_{n+1}, x_{S \cup\{n+1\}}\right)$. In subcase $(i)$ it holds that

$$
\begin{aligned}
Q_{h} & =\left(1, x_{n+1}, x_{n+1}\right)=x_{n+1}(1,1,1)+\left(1-x_{n+1}\right)(1,0,0) \\
& =x_{n+1} Q_{1}+\left(1-x_{n+1}\right) Q_{2} .
\end{aligned}
$$

In subcase $(i i)$ it holds that

$$
\begin{aligned}
Q_{h} & =\left(0, x_{n+1}, 0\right)=x_{n+1}(0,1,0)+\left(1-x_{n+1}\right)(0,0,0) \\
& =x_{n+1} Q_{3}+\left(1-x_{n+1}\right) Q_{4} .
\end{aligned}
$$

In subcase $(i i i)$, it can be verified by a finite iterative procedure that the point $Q_{h}=\left(x_{S}, x_{n+1}, x_{S \cup\{n+1\}}\right) \in$ 
$\mathcal{I}$. We examine the different cases on the cardinality $s$ of $S$. We recall that $\bigwedge_{i \in S}\left(E_{i} \mid H_{i}\right)$ is denoted by $\mathcal{C}_{S}$.

Step 1. $s=1$. Without loss of generality we assume $S=\{1\}$, so that $Q_{h}=\left(x_{S}, x_{n+1}, x_{S \cup\{n+1\}}\right)=$ $\left(x_{1}, x_{n+1}, x_{\{1, n+1\}}\right)$, where $x_{1}=P\left(E_{1} \mid H_{1}\right)$, $x_{\{1, n+1\}}=\mathbb{P}\left[\left(E_{1} \mid H_{1}\right) \wedge\left(E_{n+1} \mid H_{n+1}\right)\right]$. By Theorem 4 it holds that $\max \left\{x_{S}+x_{n+1}-\right.$ $1,0\} \leq x_{S \cup\{n+1\}} \leq \min \left\{x_{S}, x_{n+1}\right\}$, with $\left(x_{S}, x_{n+1}\right) \in[0,1]^{2}$. In other words, $Q_{h}=$ $\left(x_{S}, x_{n+1}, x_{S} \cup\{n+1\}\right) \in \mathcal{I}$. The reasoning is the same for $S=\{i\}, i=2, \ldots, n$.

Step 2. $s=2$. Without loss of generality we assume $S=\{1,2\}$, so that $x_{S}=\mathbb{P}\left[\left(E_{1} \mid H_{1}\right) \wedge\left(E_{2} \mid H_{2}\right)\right]$, $x_{S \cup\{n+1\}}=\mathbb{P}\left[\mathcal{C}_{S \cup\{n+1\}}\right]=\mathbb{P}\left[\left(E_{1} \mid H_{1}\right) \wedge\left(E_{2} \mid H_{2}\right) \wedge\right.$ $\left.\left(E_{n+1} \mid H_{n+1}\right)\right]$. We denote by $C_{0}^{*}, C_{1}^{*}, \ldots, C_{m^{*}}^{*}$, the constituents associated with $\left\{E_{i} \mid H_{i}, i \in S \cup\right.$ $\{n+1\}\}$, where $C_{0}^{*}=\bigwedge_{i \in S \cup\{n+1\}} \bar{H}_{i}$. Moreover, with $C_{h}^{*}, h=0,1, \ldots, m^{*}$, we associate the point $Q_{h}^{*}=\left(q_{h 1}^{*}, q_{h 2}^{*}, q_{h 3}^{*}\right)$ which represents the value of the random vector $\left\{\mathcal{C}_{S}, E_{n+1} \mid H_{n+1}, \mathcal{C}_{S \cup\{n+1\}}\right\}$ when $C_{h}^{*}$ is true. We observe that $Q_{0}^{*}=$ $\left(x_{S}, x_{n+1}, x_{S \cup\{n+1\}}\right)$ and that $Q_{1}, Q_{2}, Q_{3}, Q_{4}$ still belongs to the set of points $\left\{Q_{h}^{*}, h=1, \ldots, m^{*}\right\}$. In order that the assessment $\left(x_{S}, x_{n+1}, x_{S \cup\{n+1\}}\right)$ on $\left\{\mathcal{C}_{S}, E_{n+1} \mid H_{n+1}, \mathcal{C}_{S \cup\{n+1\}}\right\}$ be coherent, the point $Q_{0}^{*}=\left(x_{S}, x_{n+1}, x_{S \cup\{n+1\}}\right)$ must belong to the convex hull of points $Q_{1}^{*}, Q_{2}^{*}, \ldots, Q_{m}^{*}$. We show that for each point $Q_{h}^{*} \neq Q_{i}, i=$ $1,2,3,4$, it holds that $Q_{h}^{*} \in \mathcal{I}$. By repeating the previous reasoning we only need to analyze the subcase (iii) of case $c$ ). We have to show that, for every nonempty subset $S^{\prime} \subset S$, the point $Q_{h}^{*}=\left(x_{S^{\prime}}, x_{n+1}, x_{S^{\prime} \cup\{n+1\}}\right)$ belongs to the convex hull $\mathcal{I}$ of $Q_{1}, \ldots, Q_{4}$. As $S=\{1,2\}$, it holds that $S^{\prime}=\{1\}$, or $S^{\prime}=\{2\}$, so that $Q_{h}^{*}=$ $\left(x_{S^{\prime}}, x_{n+1}, x_{S^{\prime} \cup\{n+1\}}\right)=\left(x_{1}, x_{n+1}, x_{\{1, n+1\}}\right)$, or $Q_{h}^{*}=\left(x_{2}, x_{n+1}, x_{\{2, n+1\}}\right)$. By Step 1, in both cases $Q_{h}^{*} \in \mathcal{I}$. Thus $Q_{h}=\left(x_{S}, x_{n+1}, x_{S \cup\{n+1\}}\right) \in$ $\mathcal{I}$. In other words, $\max \left\{x_{S}+x_{n+1}-1,0\right\} \leq$ $x_{S \cup\{n+1\}} \leq \min \left\{x_{S}, x_{n+1}\right\}$, with $\left(x_{S}, x_{n+1}\right) \in$ $[0,1]^{2}$. The reasoning is the same for every $S=$ $\{i, j\} \subset\{1,2, \ldots, n\}$.

Step $k+1 . s=k+1,2<k+1<n$. By induction, assume that $\left(x_{S^{\prime}}, x_{n+1}, x_{S^{\prime} \cup\{n+1\}}\right) \in \mathcal{I}$ for every $S^{\prime}=\left\{i_{1}, i_{2}, \ldots, i_{k}\right\} \subset\{1,2, \ldots, n\}$. Then, by the previous reasoning, it follows that $Q_{h}=\left(x_{S}, x_{n+1}, x_{S \cup\{n+1\}}\right) \in \mathcal{I}$ for every $S=\left\{i_{1}, i_{2}, \ldots, i_{k+1}\right\}$. In other words, $\max \left\{x_{S}+\right.$ $\left.x_{n+1}-1,0\right\} \leq x_{S \cup\{n+1\}} \leq \min \left\{x_{S}, x_{n+1}\right\}$, with $\left(x_{S}, x_{n+1}\right) \in[0,1]^{2}$, for every $S=$ $\left\{i_{1}, i_{2}, \ldots, i_{k+1}\right\}$.
Thus, by this iterative procedure, also in the subcase (iii) of case $c$ ) it holds that $Q_{h} \in \mathcal{I}$. Then, $Q_{h} \in \mathcal{I}$, $h=5, \ldots, m$. Finally, the condition (45) is equivalent to $\mathcal{M} \in \mathcal{I}$, so that the assessment $\mathcal{M}$ is coherent if and only if

$$
\begin{aligned}
& \left(\mu_{n}, x_{n+1}\right) \in[0,1]^{2}, \quad \max \left\{\mu_{n}+x_{n+1}-1,0\right\} \leq \mu_{n+1} \\
& \quad \leq \min \left\{\mu_{n}, x_{n+1}\right\} .
\end{aligned}
$$

Proof of Theorem 15 The computation of the set $\Pi$ is based on Section 2.2. The constituents $C_{h}$ 's and the points $Q_{h}$ 's associated with $(\mathcal{F}, \mathcal{M})$ are illustrated in Table 1.

We recall that $Q_{h}=\left(q_{h 1}, \ldots, q_{h 7}\right)$ represents the value associated with $C_{h}$ of the random vector $\left(E_{1}\left|H_{1}, E_{2}\right| H_{2}\right.$, $E_{3} \mid H_{3},\left(E_{1} \mid H_{1}\right) \wedge\left(E_{2} \mid H_{2}\right),\left(E_{1} \mid H_{1}\right) \wedge\left(E_{3} \mid H_{3}\right),\left(E_{2} \mid H_{2}\right) \wedge$ $\left.\left(E_{3} \mid H_{3}\right),\left(E_{1} \mid H_{1}\right) \wedge\left(E_{2} \mid H_{2}\right) \wedge\left(E_{3} \mid H_{3}\right)\right), h=1, \ldots, 26$. With $C_{0}=\overline{H_{1}} \overline{H_{2}} \overline{H_{3}}$ it is associated $Q_{0}=\mathcal{M}$. Denoting by $\mathcal{I}$ the convex hull generated by $Q_{1}, Q_{2}, \ldots, Q_{26}$, the coherence of the prevision assessment $\mathcal{M}$ on $\mathcal{F}$ requires that the condition $\mathcal{M} \in \mathcal{I}$ be satisfied; this amounts to the solvability of the following system

$$
\begin{aligned}
\mathcal{M} & =\sum_{h=1}^{26} \lambda_{h} Q_{h}, \quad \sum_{h=1}^{26} \lambda_{h}=1, \\
\lambda_{h} & \geq 0, h=1, \ldots, 26 .
\end{aligned}
$$

We observe that

$$
\begin{aligned}
Q_{3}= & x_{3} Q_{1}+\left(1-x_{3}\right) Q_{2}, \quad Q_{6}=x_{3} Q_{4}+\left(1-x_{3}\right) Q_{5}, \\
Q_{7}= & x_{2} Q_{1}+\left(1-x_{2}\right) Q_{4}, \quad Q_{8}=x_{2} Q_{2}+\left(1-x_{2}\right) Q_{5}, \\
Q_{9}= & x_{23} Q_{1}+\left(x_{2}-x_{23}\right) Q_{2}+\left(x_{3}-x_{23}\right) Q_{4} \\
& +\left(x_{23}-x_{2}-x_{3}+1\right) Q_{5}, \\
Q_{12}= & x_{3} Q_{10}+\left(1-x_{3}\right) Q_{11}, \\
Q_{15}= & x_{3} Q_{13}+\left(1-x_{3}\right) Q_{14}, \\
Q_{16}= & x_{2} Q_{10}+\left(1-x_{2}\right) Q_{13}, \\
Q_{17}= & x_{2} Q_{11}+\left(1-x_{2}\right) Q_{14}, \\
Q_{18}= & x_{23} Q_{10}+\left(x_{2}-x_{23}\right) Q_{11}+\left(x_{3}-x_{23}\right) Q_{13} \\
& +\left(x_{23}-x_{2}-x_{3}+1\right) Q_{14}, \\
Q_{19}= & x_{1} Q_{1}+\left(1-x_{1}\right) Q_{10}, \quad Q_{20}=x_{1} Q_{2}+\left(1-x_{1}\right) Q_{11}, \\
Q_{21}= & x_{13} Q_{1}+\left(x_{1}-x_{13}\right) Q_{2}+\left(x_{3}-x_{13}\right) Q_{10} \\
& +\left(x_{13}-x_{1}-x_{3}+1\right) Q_{11}, \\
Q_{22}= & x_{1} Q_{4}+\left(1-x_{1}\right) Q_{13}, \quad Q_{23}=x_{1} Q_{5}+\left(1-x_{1}\right) Q_{14}, \\
Q_{24}= & x_{13} Q_{4}+\left(x_{1}-x_{13}\right) Q_{5}+\left(x_{3}-x_{13}\right) Q_{13} \\
& +\left(x_{13}-x_{1}-x_{3}+1\right) Q_{14}, \\
Q_{25}= & x_{12} Q_{1}+\left(x_{1}-x_{12}\right) Q_{4}+\left(x_{2}-x_{12}\right) Q_{10} \\
& +\left(x_{12}-x_{1}-x_{2}+1\right) Q_{13},
\end{aligned}
$$




$$
\begin{aligned}
Q_{26}= & x_{12} Q_{2}+\left(x_{1}-x_{12}\right) Q_{5}+\left(x_{2}-x_{12}\right) Q_{11} \\
& +\left(x_{12}-x_{1}-x_{2}+1\right) Q_{14} .
\end{aligned}
$$

Thus, $\mathcal{I}$ coincides with the convex hull of the points $Q_{1}, Q_{2}$, $Q_{4}, Q_{5}, Q_{10}, Q_{11}, Q_{13}, Q_{14}$. For the sake of simplicity, we set: $Q_{1}^{\prime}=Q_{1}, Q_{2}^{\prime}=Q_{2}, Q_{3}^{\prime}=Q_{4}, Q_{4}^{\prime}=Q_{5}, Q_{5}^{\prime}=Q_{10}$, $Q_{6}^{\prime}=Q_{11}, Q_{7}^{\prime}=Q_{13}, Q_{8}^{\prime}=Q_{14}$. Then, the condition $\mathcal{M} \in \mathcal{I}$ amounts to the solvability of the following system

$$
\mathcal{M}=\sum_{\substack{8=1 \\ \lambda_{h}^{\prime} \geq 0, h=1, \ldots, 8}}^{\lambda_{h}^{\prime} Q_{h}^{\prime}, \quad \sum_{h=1}^{8} \lambda_{h}^{\prime}=1,}
$$

that is

$\left(\Sigma^{\prime}\right)\left\{\begin{array}{l}\lambda_{1}^{\prime}+\lambda_{2}^{\prime}+\lambda_{3}^{\prime}+\lambda_{4}^{\prime}=x_{1}, \quad \lambda_{1}^{\prime}+\lambda_{2}^{\prime}+\lambda_{5}^{\prime}+\lambda_{6}^{\prime}=x_{2}, \\ \lambda_{1}^{\prime}+\lambda_{3}^{\prime}+\lambda_{5}^{\prime}+\lambda_{7}^{\prime}=x_{3}, \quad \lambda_{1}^{\prime}+\lambda_{2}^{\prime}=x_{12}, \\ \lambda_{1}^{\prime}+\lambda_{3}^{\prime}=x_{13}, \quad \lambda_{1}^{\prime}+\lambda_{5}^{\prime}=x_{23}, \quad \lambda_{1}^{\prime}=x_{123}, \\ \sum_{h=1}^{8} \lambda_{h}^{\prime}=1, \quad \lambda_{h}^{\prime} \geq 0, \quad h=1,2, \ldots, 8 .\end{array}\right.$

System $\left(\Sigma^{\prime}\right)$ can be written as

$\left(\Sigma^{\prime}\right)\left\{\begin{array}{l}\lambda_{1}^{\prime}=x_{123}, \quad \lambda_{2}^{\prime}=x_{12}-x_{123}, \\ \lambda_{3}^{\prime}=x_{13}-x_{123}, \quad \lambda_{4}^{\prime}=x_{1}-x_{12}-x_{13}+x_{123}, \\ \lambda_{5}^{\prime}=x_{23}-x_{123}, \quad \lambda_{6}^{\prime}=x_{2}-x_{12}-x_{23}+x_{123}, \\ \lambda_{7}^{\prime}=x_{3}-x_{13}-x_{23}+x_{123}, \\ \lambda_{8}^{\prime}=1-x_{1}-x_{2}-x_{3}+x_{12}+x_{13}+x_{23}-x_{123}, \\ \lambda_{h}^{\prime} \geq 0, \quad h=1,2, \ldots, 8 .\end{array}\right.$

As it can be verified, by non-negativity of $\lambda_{1}^{\prime}, \ldots, \lambda_{8}^{\prime}$ it follows that $\left(\Sigma^{\prime}\right)$ is solvable (with a unique solution) if and only if

$\left\{\begin{array}{l}x_{123} \geq \max \left\{0, x_{12}+x_{13}-x_{1}, x_{12}+x_{23}-x_{2}, x_{13}+x_{23}-x_{3}\right\}, \\ x_{123} \leq \min \left\{x_{12}, x_{13}, x_{23}, 1-x_{1}-x_{2}-x_{3}+x_{12}+x_{13}+x_{23}\right\},\end{array}\right.$

or, in a more explicit way, if and only if the following conditions are satisfied

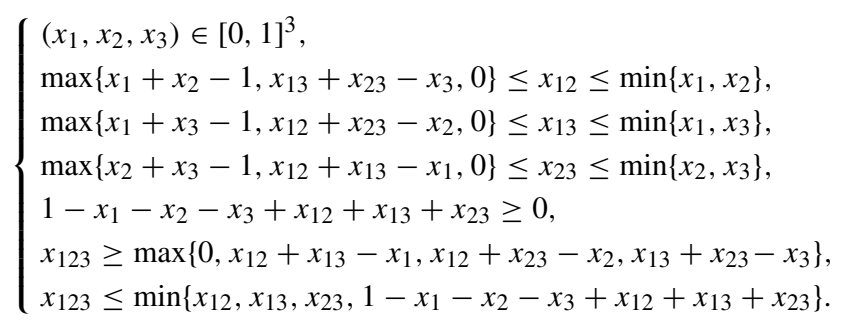

Notice that the conditions in (47) coincide with that ones in (18). Moreover, assuming $\left(\Sigma^{\prime}\right)$ solvable, with the solution $\left(\lambda_{1}^{\prime}, \ldots, \lambda_{8}^{\prime}\right)$, we associate the vector $\left(\lambda_{1}, \lambda_{2}, \ldots, \lambda_{26}\right)$, with $\lambda_{1}=\lambda_{1}^{\prime}, \quad \lambda_{2}=\lambda_{2}^{\prime}, \quad \lambda_{4}=\lambda_{3}^{\prime}, \quad \lambda_{5}=\lambda_{4}^{\prime}, \quad \lambda_{10}=$ $\lambda_{5}^{\prime}, \quad \lambda_{11}=\lambda_{6}^{\prime}, \quad \lambda_{13}=\lambda_{7}^{\prime}, \quad \lambda_{14}=\lambda_{8}^{\prime}, \quad \lambda_{h}=0, h \notin$ $\{1,2,4,5,10,11,13,14\}$, which is a solution of $(\Sigma)$. Moreover, defining $\mathcal{J}=\{1,2,4,5,10,11,13,14\}$, it holds that $\bigvee_{h \in \mathcal{J}} C_{h}=H_{1} \wedge H_{2} \wedge H_{3}$. Therefore, $\sum_{h \in \mathcal{J}} \lambda_{h}=$ $\sum_{h: C_{h} \subseteq H_{1} H_{2} H_{3}} \lambda_{h}=1$ and hence $\sum_{h: C_{h} \subseteq H_{i}} \lambda_{h}=$ $1, i=1,2,3, \sum_{h: C_{h} \subseteq H_{i} \vee H_{j}} \lambda_{h}=1, i \neq j$, $\sum_{h: C_{h} \subseteq H_{1} \vee H_{2} \vee H_{3}} \lambda_{h}=1$; thus, by (2), $I_{0}=\emptyset$. Then, by Theorem 2, the solvability of $(\Sigma)$ is also sufficient for the coherence of $\mathcal{M}$. Finally, $\Pi$ is the set of conditional prevision assessments $\left(x_{1}, x_{2}, x_{3}, x_{12}, x_{13,23}, x_{123}\right)$ which satisfy the conditions in (18).

Proof of Theorem 16 Notice that, $\left(E_{i} \mid H\right) \wedge\left(E_{j} \mid H\right)=$ $\left(E_{i} E_{j}\right) \mid H$, for every $\{i, j\} \subset\{1,2,3\}$, and $\left(E_{1} \mid H\right) \wedge\left(E_{2} \mid H\right) \wedge\left(E_{3} \mid H\right)=\left(E_{1} E_{2} E_{3}\right) \mid H$. Then $\mathcal{F}=$ $\left\{E_{1}\left|H, E_{2}\right| H, E_{3}\left|H,\left(E_{1} E_{2}\right)\right| H,\left(E_{1} E_{3}\right)\left|H,\left(E_{2} E_{3}\right)\right| H\right.$, $\left.\left(E_{1} E_{2} E_{3}\right) \mid H\right\}$. The computation of the set $\Pi$ is based on Section 2.2. The constituents $C_{h}$ 's and the points $Q_{h}$ 's associated with $(\mathcal{F}, \mathcal{M})$ are illustrated in Table 2.

We recall that $Q_{h}=\left(q_{h 1}, \ldots, q_{h 7}\right)$ represents the value associated with $C_{h}$ of the random vector $\left(E_{1}\left|H, E_{2}\right| H, E_{3}\left|H,\left(E_{1} E_{2}\right)\right| H, \quad\left(E_{1} E_{3}\right)\left|H,\left(E_{2} E_{3}\right)\right| H\right.$, $\left.\left(E_{1} E_{2} E_{3}\right) \mid H\right), h=1, \ldots, 8$. With $C_{0}=\bar{H}$ it is associated $Q_{0}=\mathcal{M}$. Denoting by $\mathcal{I}$ the convex hull generated by $Q_{1}, Q_{2}, \ldots, Q_{8}$, as all the conditioning events coincide with $H$ the assessment $\mathcal{M}$ on $\mathcal{F}$ is coherent if and only if $\mathcal{M} \in \mathcal{I}$; that is, if and only if the following system is solvable

$\mathcal{M}=\sum_{h=1}^{8} \lambda_{h} Q_{h}, \quad \sum_{h=1}^{8} \lambda_{h}=1, \quad \lambda_{h} \geq 0, \quad h=1, \ldots, 8$.

The points $Q_{1}, Q_{2}, \ldots, Q_{8}$ coincide with the points $Q_{1}^{\prime}, Q_{2}^{\prime}, \ldots, Q_{8}^{\prime}$ in the proof of Theorem 15 , respectively. Then, system (48) coincides with system $(\Sigma)^{\prime}$ in the proof of Theorem 15. Therefore, it is solvable if and only if the conditions in (18) are satisfied. In other words, the set $\Pi$ of all coherent assessments $\mathcal{M}$ on $\mathcal{F}$ coincides with the set of points $\left(x_{1}, x_{2}, x_{3}, x_{12}, x_{13}, x_{23}, x_{123}\right)$ which satisfy the conditions in (18).

Proof of of Theorem 18 In order to prove the theorem it is enough to prove the following implications: a) $(i) \Rightarrow(i i)$; b) $(i i) \Rightarrow(i i i) ;$ c) $($ iii $) \Rightarrow(i)$.

a) (i) $\Rightarrow$ (ii). We recall that $\mathcal{F}$ p-entails $E_{n+1} \mid H_{n+1}$ if and only if either $H_{n+1} \subseteq E_{n+1}$, or there exists a nonempty $\mathcal{F}_{\Gamma} \subseteq \mathcal{F}$, where $\Gamma \subseteq\{1, \ldots, n\}$, such that $Q C\left(\mathcal{F}_{\Gamma}\right)$ implies $E_{n+1} \mid H_{n+1}$ (see, e.g. [24, Theorem 6]). Let us first consider the case where $H_{n+1} \subseteq E_{n+1}$. In this case $P\left(E_{n+1} \mid H_{n+1}\right)=1$ and $E_{n+1} \mid H_{n+1}=$ $H_{n+1}+\bar{H}_{n+1}=1$. We have $\mathcal{C}_{n+1}=\mathcal{C}_{n} \wedge\left(E_{n+1} \mid H_{n+1}\right)$, with $E_{n+1} \mid H_{n+1}=1$. We distinguish two cases: 
Table 1 Constituents $C_{h}$ 's and corresponding points $Q_{h}$ 's associated with $(\mathcal{F}, \mathcal{M})$, where $\mathcal{M}=\left(x_{1}, x_{2}, x_{3}, x_{12}, x_{13}, x_{23}, x_{123}\right)$ is a prevision assessment on $\mathcal{F}=\left\{E_{1}\left|H_{1}, E_{2}\right| H_{2}, E_{3} \mid H_{3},\left(E_{1} \mid H_{1}\right) \wedge\left(E_{2} \mid H_{2}\right),\left(E_{1} \mid H_{1}\right) \wedge\left(E_{3} \mid H_{3}\right),\left(E_{2} \mid H_{2}\right) \wedge\left(E_{3} \mid H_{3}\right),\left(E_{1} \mid H_{1}\right) \wedge\left(E_{2} \mid H_{2}\right) \wedge\left(E_{3} \mid H_{3}\right)\right\}$

\begin{tabular}{|c|c|c|c|c|c|c|c|c|c|}
\hline & $C_{h}$ & $Q_{h}$ & & & & & & & \\
\hline$C_{1}$ & $E_{1} H_{1} E_{2} H_{2} E_{3} H_{3}$ & 1 & 1 & 1 & 1 & 1 & 1 & 1 & $Q_{1}$ \\
\hline$C_{2}$ & $E_{1} H_{1} E_{2} H_{2} \bar{E}_{3} H_{3}$ & 1 & 1 & 0 & 1 & 0 & 0 & 0 & $Q_{2}$ \\
\hline$C_{3}$ & $E_{1} H_{1} E_{2} H_{2} \bar{H}_{3}$ & 1 & 1 & $x_{3}$ & 1 & $x_{3}$ & $x_{3}$ & $x_{3}$ & $Q_{3}$ \\
\hline$C_{4}$ & $E_{1} H_{1} \bar{E}_{2} H_{2} E_{3} H_{3}$ & 1 & 0 & 1 & 0 & 1 & 0 & 0 & $Q_{4}$ \\
\hline$C_{5}$ & $E_{1} H_{1} \bar{E}_{2} H_{2} \bar{E}_{3} H_{3}$ & 1 & 0 & 0 & 0 & 0 & 0 & 0 & $Q_{5}$ \\
\hline$C_{6}$ & $E_{1} H_{1} \bar{E}_{2} H_{2} \bar{H}_{3}$ & 1 & 0 & $x_{3}$ & 0 & $x_{3}$ & 0 & 0 & $Q_{6}$ \\
\hline$C_{7}$ & $E_{1} H_{1} \bar{H}_{2} E_{3} H_{3}$ & 1 & $x_{2}$ & 1 & $x_{2}$ & 1 & $x_{2}$ & $x_{2}$ & $Q_{7}$ \\
\hline$C_{8}$ & $E_{1} H_{1} \bar{H}_{2} \bar{E}_{3} H_{3}$ & 1 & $x_{2}$ & 0 & $x_{2}$ & 0 & 0 & 0 & $Q_{8}$ \\
\hline$C_{9}$ & $E_{1} H_{1} \bar{H}_{2} \bar{H}_{3}$ & 1 & $x_{2}$ & $x_{3}$ & $x_{2}$ & $x_{3}$ & $x_{23}$ & $x_{23}$ & $Q_{9}$ \\
\hline$C_{10}$ & $\bar{E}_{1} H_{1} E_{2} H_{2} E_{3} H_{3}$ & 0 & 1 & 1 & 0 & 0 & 1 & 0 & $Q_{10}$ \\
\hline$C_{11}$ & $\bar{E}_{1} H_{1} E_{2} H_{2} \bar{E}_{3} H_{3}$ & 0 & 1 & 0 & 0 & 0 & 0 & 0 & $Q_{11}$ \\
\hline$C_{12}$ & $\bar{E}_{1} H_{1} E_{2} H_{2} \bar{H}_{3}$ & 0 & 1 & $x_{3}$ & 0 & 0 & $x_{3}$ & 0 & $Q_{12}$ \\
\hline$C_{13}$ & $\bar{E}_{1} H_{1} \bar{E}_{2} H_{2} E_{3} H_{3}$ & 0 & 0 & 1 & 0 & 0 & 0 & 0 & $Q_{13}$ \\
\hline$C_{14}$ & $\bar{E}_{1} H_{1} \bar{E}_{2} H_{2} \bar{E}_{3} H_{3}$ & 0 & 0 & 0 & 0 & 0 & 0 & 0 & $Q_{14}$ \\
\hline$C_{15}$ & $\bar{E}_{1} H_{1} \bar{E}_{2} H_{2} \bar{H}_{3}$ & 0 & 0 & $x_{3}$ & 0 & 0 & 0 & 0 & $Q_{15}$ \\
\hline$C_{16}$ & $\bar{E}_{1} H_{1} \bar{H}_{2} E_{3} H_{3}$ & 0 & $x_{2}$ & 1 & 0 & 0 & $x_{2}$ & 0 & $Q_{16}$ \\
\hline$C_{17}$ & $\bar{E}_{1} H_{1} \bar{H}_{2} \bar{E}_{3} H_{3}$ & 0 & $x_{2}$ & 0 & 0 & 0 & 0 & 0 & $Q_{17}$ \\
\hline$C_{18}$ & $\bar{E}_{1} H_{1} \bar{H}_{2} \bar{H}_{3}$ & 0 & $x_{2}$ & $x_{3}$ & 0 & 0 & $x_{23}$ & 0 & $Q_{18}$ \\
\hline$C_{19}$ & $\bar{H}_{1} E_{2} H_{2} E_{3} H_{3}$ & $x_{1}$ & 1 & 1 & $x_{1}$ & $x_{1}$ & 1 & $x_{1}$ & $Q_{19}$ \\
\hline$C_{20}$ & $\bar{H}_{1} E_{2} H_{2} \bar{E}_{3} H_{3}$ & $x_{1}$ & 1 & 0 & $x_{1}$ & 0 & 0 & 0 & $Q_{20}$ \\
\hline$C_{21}$ & $\bar{H}_{1} E_{2} H_{2} \bar{H}_{3}$ & $x_{1}$ & 1 & $x_{3}$ & $x_{1}$ & $x_{13}$ & $x_{3}$ & $x_{13}$ & $Q_{21}$ \\
\hline$C_{22}$ & $\bar{H}_{1} \bar{E}_{2} H_{2} E_{3} H_{3}$ & $x_{1}$ & 0 & 1 & 0 & $x_{1}$ & 0 & 0 & $Q_{22}$ \\
\hline$C_{23}$ & $\bar{H}_{1} \bar{E}_{2} H_{2} \bar{E}_{3} H_{3}$ & $x_{1}$ & 0 & 0 & 0 & 0 & 0 & 0 & $Q_{23}$ \\
\hline$C_{24}$ & $\bar{H}_{1} \bar{E}_{2} H_{2} \bar{H}_{3}$ & $x_{1}$ & 0 & $x_{3}$ & 0 & $x_{13}$ & 0 & 0 & $Q_{24}$ \\
\hline$C_{25}$ & $\bar{H}_{1} \bar{H}_{2} E_{3} H_{3}$ & $x_{1}$ & $x_{2}$ & 1 & $x_{12}$ & $x_{1}$ & $x_{2}$ & $x_{12}$ & $Q_{25}$ \\
\hline$C_{26}$ & $\bar{H}_{1} \bar{H}_{2} \bar{E}_{3} H_{3}$ & $x_{1}$ & $x_{2}$ & 0 & $x_{12}$ & 0 & 0 & 0 & $Q_{26}$ \\
\hline$C_{0}$ & $\bar{H}_{1} \bar{H}_{2} \bar{H}_{3}$ & $x_{1}$ & $x_{2}$ & $x_{3}$ & $x_{12}$ & $x_{13}$ & $x_{23}$ & $x_{123}$ & $Q_{0}$ \\
\hline
\end{tabular}

( $\alpha) H_{n+1}$ is true; $(\beta) H_{n+1}$ is false. In case $(\alpha)$, by Definition 5 and Remark 4, as $E_{n+1} \mid H_{n+1}$ is true it follows that the values of $\mathcal{C}_{n+1}$ and of $\mathcal{C}_{n}$ coincide. In case $(\beta)$, let $C_{0}, \ldots, C_{m}$ be the constituents associated with $\mathcal{F}$, where $C_{0}=\bar{H}_{1} \cdots \bar{H}_{n}$. Then, the constituents $C_{0}^{\prime}, \ldots, C_{m}^{\prime}$ associated with $\mathcal{F} \cup\left\{E_{n+1} \mid H_{n+1}\right\}$ and

Table 2 Constituents $C_{h}$ 's and corresponding points $Q_{h}$ 's associated with $(\mathcal{F}, \mathcal{M})$, where $\mathcal{M}=\left(x_{1}, x_{2}, x_{3}, x_{12}, x_{13}, x_{23}, x_{123}\right)$ is a prevision assessment on $\mathcal{F}=\left\{E_{1}\left|H, E_{2}\right| H, E_{3}\left|H,\left(E_{1} E_{2}\right)\right| H,\left(E_{1} E_{3}\right)\left|H,\left(E_{2} E_{3}\right)\right| H,\left(E_{1} E_{2} E_{3}\right) \mid H\right\}$

\begin{tabular}{llllllllll}
\hline \multicolumn{1}{c}{$C_{h}$} & $Q_{h}$ & & & & & & \\
\hline$C_{1}$ & $E_{1} E_{2} E_{3} H$ & 1 & 1 & 1 & 1 & 1 & 1 & 1 & $Q_{1}$ \\
$C_{2}$ & $E_{1} E_{2} \bar{E}_{3} H$ & 1 & 1 & 0 & 1 & 0 & 0 & 0 & $Q_{2}$ \\
$C_{3}$ & $E_{1} E_{2} E_{3} H$ & 1 & 0 & 1 & 0 & 1 & 0 & 0 & $Q_{3}$ \\
$C_{4}$ & $E_{1} \bar{E}_{2} \bar{E}_{3} H$ & 1 & 0 & 0 & 0 & 0 & 0 & 0 & $Q_{4}$ \\
$C_{5}$ & $\bar{E}_{1} E_{2} E_{3} H$ & 0 & 1 & 1 & 0 & 0 & 1 & 0 & $Q_{5}$ \\
$C_{6}$ & $\bar{E}_{1} E_{2} \bar{E}_{3} H$ & 0 & 1 & 0 & 0 & 0 & 0 & 0 & $Q_{6}$ \\
$C_{7}$ & $\bar{E}_{1} \bar{E}_{2} E_{3} H$ & 0 & 0 & 1 & 0 & 0 & 0 & 0 & $Q_{7}$ \\
$C_{8}$ & $\bar{E}_{1} \bar{E}_{2} \bar{E}_{3} H$ & 0 & 0 & 0 & 0 & 0 & 0 & 0 & $Q_{8}$ \\
$C_{0}$ & $\bar{H}$ & $x_{1}$ & $x_{2}$ & $x_{3}$ & $x_{12}$ & $x_{13}$ & $x_{23}$ & $x_{123}$ & $Q_{0}$ \\
\hline
\end{tabular}


contained in $\bar{H}_{n+1}$ are $C_{0}^{\prime}=C_{0} \bar{H}_{n+1}, \ldots, C_{m}^{\prime}=$ $C_{m} \bar{H}_{n+1}$. For each constituent $C_{h}^{\prime}, h=1, \ldots, m$, by formula (10) the corresponding value of $\mathcal{C}_{n}$ is $z_{h} \in\left\{1,0, x_{S_{h}^{\prime \prime \prime}}\right\}$. We denote by $z_{h}^{\prime}$ the value of $\mathcal{C}_{n+1}$ associated with $z_{h}$ and we recall that $C_{h}^{\prime} \subseteq \bar{H}_{n+1}$, $h=0,1, \ldots, m$. For each index $h$, if $z_{h}=1$, then $z_{h}^{\prime}=1$; if $z_{h}=0$, then $z_{h}^{\prime}=0$; if $z_{h}=x_{S_{h}^{\prime \prime \prime}}$, then $z_{h}^{\prime}=x_{S_{h}^{\prime \prime \prime} \cup\{n+1\}}$. We set $P\left(E_{n+1} \mid H_{n+1}\right)=x_{n+1}$; in our case $x_{n+1}=1$. Moreover, by Theorem 10

$\max \left\{x_{S_{h}^{\prime \prime \prime}}+x_{n+1}-1,0\right\} \leq x_{S_{h}^{\prime \prime \prime} \cup\{n+1\}} \leq \min \left\{x_{S_{h}^{\prime \prime \prime}}, x_{n+1}\right\} ;$

therefore $x_{S_{h}^{\prime \prime \prime} \cup\{n+1\}}=x_{S_{h}^{\prime \prime \prime}}$. Then, the values of $\mathcal{C}_{n+1}$ and of $\mathcal{C}_{n}$ coincide for every $C_{h}^{\prime}$. Thus, $\mathcal{C}_{n+1}=\mathcal{C}_{n}$ when $H_{n+1} \subseteq E_{n+1}$.

We consider now the case where there exists $\mathcal{F}_{\Gamma} \subseteq$ $\mathcal{F}, \mathcal{F}_{\Gamma} \neq \emptyset$, such that $Q C\left(\mathcal{F}_{\Gamma}\right) \subseteq E_{n+1} \mid H_{n+1}$. First of all we prove that $\mathcal{C}\left(\mathcal{F}_{\Gamma} \cup\left\{E_{n+1} \mid H_{n+1}\right\}\right)=\mathcal{C}\left(\mathcal{F}_{\Gamma}\right)$. For the sake of simplicity, we set $\mathcal{C}\left(\mathcal{F}_{\Gamma}\right)=\mathcal{C}_{\Gamma}$ and $\mathcal{C}\left(\mathcal{F}_{\Gamma} \cup\left\{E_{n+1} \mid H_{n+1}\right\}\right)=\mathcal{C}_{\Gamma \cup\{n+1\}}$.

If the value of $\mathcal{C}_{\Gamma}$ is 1 (because all the conditional events in $\mathcal{F}_{\Gamma}$ are true), then $Q C\left(\mathcal{F}_{\Gamma}\right)$ is true and hence $E_{n+1} \mid H_{n+1}$ is also true; thus $\mathcal{C}_{\Gamma \cup\{n+1\}}=1$, so that $\mathcal{C}_{\Gamma \cup\{n+1\}}=\mathcal{C}_{\Gamma}$.

If the value of $\mathcal{C}_{\Gamma}$ is 0 (because some conditional event in $\mathcal{F}_{\Gamma}$ is false), then $\mathcal{C}_{\Gamma \cup\{n+1\}}$ is 0 too, so that $\mathcal{C}_{\Gamma \cup\{n+1\}}=\mathcal{C}_{\Gamma}$.

If $\mathcal{C}_{\Gamma}$ is $x_{S}$ for some nonempty subset $\mathcal{S} \subset \Gamma$ (that is, all the conditional events in $\mathcal{F}_{S}$ are void and the other ones in $\mathcal{F}_{\Gamma \backslash S}$ are true), then $Q C\left(\mathcal{F}_{\Gamma}\right)$ is true and and hence $E_{n+1} \mid H_{n+1}$ is also true; thus $\mathcal{C}_{\Gamma \cup\{n+1\}}=x_{S}$, so that $\mathcal{C}_{\Gamma \cup\{n+1\}}=\mathcal{C}_{\Gamma}$.

If $\mathcal{C}_{\Gamma}$ is $x_{\Gamma}$ because all the conditional events in $\mathcal{F}_{\Gamma}$ are void, then $Q C\left(\mathcal{F}_{\Gamma}\right)$ is void and for $E_{n+1} \mid H_{n+1}$ there are two cases: 1) $E_{n+1} \mid H_{n+1}$ true; 2) $E_{n+1} \mid H_{n+1}$ void. In case 1), by also recalling Remark 4 , it holds that $\mathcal{C}_{\Gamma \cup\{n+1\}}=x_{\Gamma}$ so that $\mathcal{C}_{\Gamma \cup\{n+1\}}=\mathcal{C}_{\Gamma}$.

In case 2) it holds that $\mathcal{C}_{\Gamma \cup\{n+1\}}=x_{\Gamma \cup\{n+1\}}$, where $x_{\Gamma \cup\{n+1\}}=\mathbb{P}\left(\mathcal{C}_{\Gamma \cup\{n+1\}}\right)$. Now, we observe that the random quantities $\mathcal{C}_{\Gamma}$ and $\mathcal{C}_{\Gamma \cup\{n+1\}}$ coincide conditionally on $\bigvee_{i \in \Gamma \cup\{n+1\}} H_{i}$ being true; then by Theorem 3 it holds that $\mathbb{P}\left(\mathcal{C}_{\Gamma}\right)=\mathbb{P}\left(\mathcal{C}_{\Gamma \cup\{n+1\}}\right)$, that is $x_{\Gamma}=x_{\Gamma \cup\{n+1\}}$; thus $\mathcal{C}_{\Gamma \cup\{n+1\}}=\mathcal{C}_{\Gamma}$.

Finally, denoting by $\Gamma^{c}$ the set $\{1, \ldots, n\} \backslash \Gamma$, by the associative property of conjunction we obtain

$$
\begin{aligned}
\mathcal{C}_{n+1} & =\mathcal{C}_{n} \wedge E_{n+1}\left|H_{n+1}=\mathcal{C}_{\Gamma^{c}} \wedge \mathcal{C}_{\Gamma} \wedge E_{n+1}\right| H_{n+1} \\
& =\mathcal{C}_{\Gamma^{c}} \wedge \mathcal{C}_{\Gamma}=\mathcal{C}_{n} .
\end{aligned}
$$

b) (ii) $\Rightarrow$ (iii). By monotonicity property of conjunction it holds that $\mathcal{C}_{n+1} \leq E_{n+1} \mid H_{n+1}$. Then, by assuming $\mathcal{C}_{n}=\mathcal{C}_{n+1}$, it follows $\mathcal{C}_{n} \leq E_{n+1} \mid H_{n+1}$.

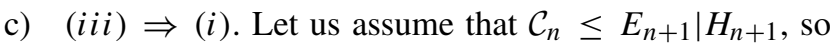
that $\mathbb{P}\left(\mathcal{C}_{n}\right) \leq P\left(E_{n+1} \mid H_{n+1}\right)$. Moreover, by assuming that $P\left(E_{i} \mid H_{i}\right)=1, i=1, \ldots, n$, from (15) it follows $\mathbb{P}\left(\mathcal{C}_{n}\right)=1$ and hence $P\left(E_{n+1} \mid H_{n+1}\right)=1$, that is $\mathcal{F}$ p-entails $E_{n+1} \mid H_{n+1}$.

\section{References}

1. Adams EW (1965) The logic of conditionals. Inquiry 8:166-197

2. Adams EW (1975) The logic of conditionals. Reidel, Dordrecht

3. Benferhat S, Dubois D, Prade H (1997) Nonmonotonic reasoning, conditional objects and possibility theory. Artif Intell 92:259-276

4. Biazzo V, Gilio A (2000) A generalization of the fundamental theorem of de Finetti for imprecise conditional probability assessments. Int J Approx Reason 24(2-3):251-272

5. Biazzo V, Gilio A, Lukasiewicz T, Sanfilippo G (2005) Probabilistic logic under coherence: complexity and algorithms. Ann Math Artif Intell 45(1-2):35-81

6. Biazzo V, Gilio A, Sanfilippo G (2008) Generalized coherence and connection property of imprecise conditional previsions. In: Proceedings of the IPMU 2008, Malaga, Spain, June 22-27, pp 907-914

7. Biazzo V, Gilio A, Sanfilippo G (2012) Coherent conditional previsions and proper scoring rules. In: Advances in computational intelligence. IPMU 2012, volume 300 of CCIS. Springer, Berlin, pp 146-156

8. Boole G (1857) On the Application of the theory of probabilities to the question of the combination of testimonies or judgments. Trans R Soc Edinb 21(4):597-653

9. Calabrese P (1987) An algebraic synthesis of the foundations of logic and probability. Inf Sci 42(3):187-237

10. Capotorti A, Lad F, Sanfilippo G (2007) Reassessing accuracy rates of median decisions. Am Stat 61(2):132-138

11. Ciucci D, Dubois D (2012) Relationships between connectives in three-valued logics. In: Advances on computational intelligence, volume 297 of CCIS. Springer, Berlin, pp 633-642

12. Coletti G, Scozzafava R (1999) Conditioning and inference in intelligent systems. Soft Comput 3(3):118-130

13. Coletti G, Scozzafava R (2002) Probabilistic logic in a coherent setting. Kluwer, Dordrecht

14. Coletti G, Scozzafava R, Vantaggi B (2013) Coherent conditional probability, fuzzy inclusion and default rules. In: Yager RR, Abbasov AM, Reformat MZ, Shahbazova SN (eds) Soft computing: state of the art theory and novel applications. Springer, Heidelberg, pp 193-208

15. Coletti G, Scozzafava R, Vantaggi B (2015) Possibilistic and probabilistic logic under coherence: default reasoning and System P. Math Slovaca 65(4):863-890

16. de Finetti B (1936) La logique de la probabilité. In: Actes du Congrès International de Philosophie Scientifique, Paris, 1935. Hermann et C.ie, Paris, pp IV 1-IV 9

17. Edgington D (1995) On conditionals. Mind 104:235-329

18. Gilio A (2002) Probabilistic reasoning under coherence in System P. Ann Math Artif Intell 34:5-34

19. Gilio A (2012) Generalizing inference rules in a coherence-based probabilistic default reasoning. Int J Approx Reason 53(3):413434

20. Gilio A, Sanfilippo G (2010) Quasi conjunction and p-entailment in nonmonotonic reasoning. In: Combining soft computing and statistical methods in data analysis, volume 77 of Advances in intelligent and soft computing. Springer, Berlin, pp 321-328

21. Gilio A, Sanfilippo G (2011) Coherent conditional probabilities and proper scoring rules. In: Proceedings of ISIPTA'11. Innsbruck, pp 189-198

22. Gilio A, Sanfilippo G (2013) Conditional random quantities and iterated conditioning in the setting of coherence. In: van der Gaag 
LC (ed) Symbolic and Quantitative Approaches to Reasoning with Uncertainty, ECSQARU 2013, volume 7958 of LNCS. Springer, Berlin, pp 218-229

23. Gilio A, Sanfilippo G (2013) Conjunction, disjunction and iterated conditioning of conditional events. In: Synergies of soft computing and statistics for intelligent data analysis, volume 190 of AISC. Springer, Berlin, pp 399-407

24. Gilio A, Sanfilippo G (2013) Probabilistic entailment in the setting of coherence: the role of quasi conjunction and inclusion relation. Int J Approx Reason 54(4):513-525

25. Gilio A, Sanfilippo G (2013) Quasi conjunction, quasi disjunction, t-norms and t-conorms: probabilistic aspects. Inf Sci 245:146167

26. Gilio A, Sanfilippo G (2014) Conditional random quantities and compounds of conditionals. Stud Log 102(4):709-729

27. Gilio A, Sanfilippo G (2017) Conjunction and disjunction among conditional events. In: Benferhat S, Tabia K, Ali M (eds) IEA/AIE 2017, Part II, volume 10351 of LNCS. Springer, Cham, pp 85-96

28. Gilio A, Pfeifer N, Sanfilippo G (2016) Transitivity in coherencebased probability logic. J Appl Log 14:46-64

29. Gilio A, Over D, Pfeifer N, Sanfilippo G (2017) Centering and compound conditionals under coherence. In: Soft methods for data science, volume 456 of AISC. Springer, Berlin, pp 253-260

30. Gilio A, Pfeifer N, Sanfilippo G Probabilistic entailment and iterated conditionals, submitted. Available on arXiv:1804.06187v1

31. Goodman IR, Nguyen HT (1988) Conditional objects and the modeling of uncertainties. In: Gupta MM, Yamakawa T (eds) Fuzzy computing. North-Holland, pp 119-138

32. Goodman IR, Nguyen HT, Walker EA (1991) Conditional inference and logic for intelligent systems: a theory of measurefree conditioning. North-Holland, Amsterdam

33. Hailperin T (1996) Sentential probability logic. Origins, development, current status, and technical applications. Lehigh University Press, Bethlehem

34. Kaufmann S (2009) Conditionals right and left: probabilities for the whole family. J Philos Log 38:1-53

35. Lad F (1996) Operational subjective statistical methods: a mathematical, philosophical, and historical introduction. Wiley, New York

36. Lad F, Sanfilippo G, Agró G (2012) Completing the logarithmic scoring rule for assessing probability distributions. AIP Conf Proc 1490(1):13-30

37. Lad F, Sanfilippo G, Agró G (2015) Extropy: complementary dual of entropy. Stat Sci 30(1):40-58

38. McGee V (1989) Conditional probabilities and compounds of conditionals. Philos Rev 98(4):485-541

39. Milne P (1997) Bruno de Finetti and the logic of conditional events. Br J Philos Sci 48(2):195-232

40. Petturiti D, Vantaggi B (2017) Envelopes of conditional probabilities extending a strategy and a prior probability. Int $\mathrm{J}$ Approx Reason 81:160-182

41. Sanfilippo G, Pfeifer N, Gilio A (2017) Generalized probabilistic modus ponens. In: Antonucci A, Cholvy L, Papini O (eds) Symbolic and Quantitative Approaches to Reasoning with Uncertainty, ECSQARU 2017, volume 10369 of LNCS. Springer, Berlin, pp 480-490

42. Sanfilippo G, Pfeifer N, Over DE, Gilio A (2018) Probabilistic inferences from conjoined to iterated conditionals. Int J Approx Reason 93(Supplement C):103-118

43. Verheij B (2017) Proof with and without probabilities. Artif Intell Law 25(1):127-154

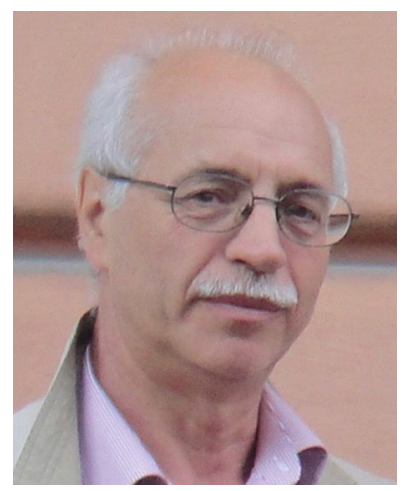

Angelo Gilio received his degree in mathematics from University of Rome "La Sapienza", Italy (1973). He obtained a research grant from CNR, Rome, Italy (1974) and a four-years contract from the Engineering Faculty of University of Rome (1976-1980). $\mathrm{He}$ has been a researcher at University of Rome "La Sapienza" from 1980 to 1994. Then, he has been full professor of probability theory at University of Catania until 2000. From 2001 to 2014 he has been full professor at University of Rome "La Sapienza"; he has been member in the scientific board in Ph.D. program in mathematical methods and models. From November 2014 he retired. His main research interests concern subjective probability, penalty criterion for conditional events, coherent conditional probability, probabilistic reasoning under coherence, imprecise probabilities, probabilistic nonmonotonic reasoning, coherence-based probabilistic approach to cognitive psychology, logical operations among conditional events.

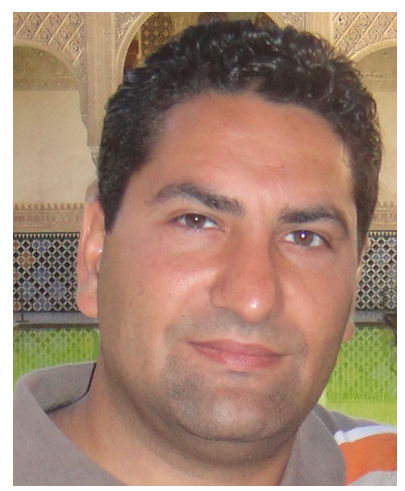

Giuseppe Sanfilippo received his degree in mathematics from University of Catania, Italy (1999). He obtained a doctorate in applied mathematics and computer science from the University of Napoli "Federico II", Italy (2004). He obtained a research grant from the University of Rome "La Sapienza", Italy in 2004. He was a visiting researcher at the INRI (Tolouse, France), at the Department of Mathematics and Statistics, Christchurch (New Zealand) and at Universitat Salzburg (Austria). He has been a researcher at University of Palermo, Italy from 2005 to 2017 . He has been one of the three coordinators of a trilateral research conferences on "Human rationality: probabilistic points of view" at Villa Vigoni, Italy (2015-2017). He is currently member in the scientific board in Ph.D. program in mathematics and computational science (Universities of Palermo, Catania and Messina). From 2018 he is associate professor of probability theory at University of Palermo.

His research interests are in subjective probability, probabilistic reasoning under coherence, coherent conditional probability, imprecise probabilities, probabilistic nonmonotonic reasoning, proper scoring rules, sequential forecasting, extropy, probabilistic square of opposition, probabilistic semantics for categorical syllogisms, logical operations among conditional events. 